\title{
A Novel Depreciation Approach in an Uncertain Environment: Interval Type-2 Fuzzy Sets in Maritime
}

\section{Ercan AKAN ( $\nabla$ ercan.akan@iste.edu.tr)}

Iskenderun Technical University: Iskenderun Teknik Universitesi https://orcid.org/0000-0003-03838290

\section{Kasım KIRACI}

Iskenderun Technical University: Iskenderun Teknik Universitesi

\section{Research Article}

Keywords: deprecation, uncertainty, interval type-2 fuzzy sets, ship's deprecation, maritime

Posted Date: November 8th, 2021

DOI: https://doi.org/10.21203/rs.3.rs-761976/v1

License: (c) (1) This work is licensed under a Creative Commons Attribution 4.0 International License. Read Full License

Version of Record: A version of this preprint was published at Soft Computing on February 24th, 2022. See the published version at https://doi.org/10.1007/s00500-022-06778-6. 


\title{
A Novel Depreciation Approach in an Uncertain Environment: Interval Type-2 Fuzzy Sets in Maritime
}

\begin{abstract}
This study aims to propose a novel model for the determination of depreciation in an environment of uncertainty. In the study, amortization methods were revised through Interval Type-2 Fuzzy (IT2FSs), and a new approach was proposed for investors to make decisions in an environment of cash flow is uncertainty. In the study, we provide options among different depreciation alternatives in the future investment decisions of maritime companies through the revision of the straight-line depreciation method and the double-declining balance depreciation method. The fuzzy depreciation alternatives we suggest in our study are not only suitable for maritime companies, but also companies in different industries.
\end{abstract}

Keywords: deprecation, uncertainty, interval type-2 fuzzy sets, ship's deprecation, maritime

\section{INTRODUCTION}

Depreciation is widely applied to protect working capital. In addition, it is related to converting the cost of the asset into expenses to ensure that the fixed asset, which loses its value over time or wears out due to use, is fully or partially covered. Depreciation is important for enabling self-financing, ensuring that depreciation expense is stretched out over accounting periods, calculating production costs, and ensuring the actual value of fixed assets (Çankaya \& Yilmaz, 2014; Engin \& Atabay, 2018). Depreciation is not only for fixed asset value but also for cash flow and market value measures of investment performance such as internal rate of return and holding period (Bokhari \& Geltner, 2018). Depreciation also allows the company's activities to be sustainable. Both cash flow forecasts and the depreciation method used are important for making the right investment decisions in capital-intensive industries. However, managers often take investment decisions in an uncertain environment. This uncertainty is often related to the estimated cash flows.

When a capital asset is purchased, its full cost does not appear in the profit and loss account. If it is considering in terms of accounting, shipping companies would have a huge loss when a ship is purchased. However, the cost of the ship in the company's balance sheet is enrolled for each year as a fixed asset, a percentage of its value is charged as a cost in the company's balance sheet for each year during the accounting period. The charge is called depreciation, not a cash value and bookkeeping in accounting, so it isn't shown in the cash flow. Also, the profit will be lower than cash flow by that amount. (IAS, 2021) doesn't mention an exactly economic useful life for ships however, in market, merchant ship's useful life is about 25-30 years is considered and some specialized ships have longer useful life so most commonly it is considered as 25 years. Therefore, equity investors face a different problem in public shipping companies. If they are a long-term investor, they focus on how much profit earned during that period so, it depends crucially on how much depreciation deducted to estimate accurately the profit earned. Hence, ship wearing out year by year during her useful life, the cost must be deducted from cash flow. Mostly used depreciation method by accountants in the shipping industry is straight-line depreciation method. However, with this study, it is presented that double-declining balance depreciation method can be easily used as interval type- 2 as much as the straight-line depreciation method. The ship is written off in defined proportions over its expected life. The proportions change depending on the type of depreciation method. The reason why the company's trading cash flow cover is so strong is that a large proportion of its costs are capital. A particularly important aspect of cash flow is the method used to pay for the ship. Many shipping companies don't purchase their ships as cash so, the ship is purchased by loan, leasing, etc. The cash flow profile of the shipping company changes due to installment and interest of the loan. When cash is tight, purchasing can be deferred and the ship traded on for a few years. The cash is plentiful, more ships can be ordered. This flexibility gives shipping companies financial security (Stopford, 2008). Nevertheless, the depreciation calculation method can give a helping solution to an investor for instance if the double- 
declining balance depreciation method than straight-line depreciation method is considered, it gives more flexibility for early years in cash flow in terms of investment. Accordingly, uncertainty the future expectations is considered can be a solution evaluated by Interval Type-2 Fuzzy Sets.

Especially due to the increasing globalization, firms have operated in a more uncertain environment. In this uncertain environment, managers need to take important decisions. Decisions are taken by managers regarding capital budgeting, capital investment, and the cost of capital affect firm value and plans. Managers take these decisions in an uncertain environment. One of the important decisions taken by managers is related to determining the depreciation method. Although it depends on accounting standards and is made on practical grounds, managers take decisions on the depreciation method in an uncertain environment. Decisions are taken by managers regarding the depreciation method affect both taxable income and shareholder value (Berg et al., 2001). Therefore, determining the depreciation method in an uncertain environment is extremely important because future cash-flows are uncertain.

Accounting principles that firms employ allow them to use different depreciation methods. There are some studies, examining the choice of the depreciation method reducing the present value of tax payments, in the literature (Jennergren, 2018; Kulp \& Hartman, 2011; Press \& Davidson, 1964; Wagenhofer, 2003). However, there are a few studies, determining which depreciation method is more appropriate in an environment where future cash flows are uncertain, in the literature. In this context, some studies in the literature suggest optimal depreciation methods in environments where cash flows are uncertain (Berg et al., 2001; Berg \& Moore, 1989). In these studies, the optimal depreciation method is proposed in different taxation systems. The main question here is whether a model can be put forward in which depreciation itself is uncertain, such as cash flows.

The various decisions that managers take in uncertain environments are important for firms to survive and gain a competitive advantage. As mentioned before, the constant depreciation amount significantly affects firms ' capital investment decisions in times when the number of future cash flows is uncertain (Jackson, 2008; Jackson et al., 2009; Ohrn, 2019). Managers take investment decisions when there is a large imbalance between productivity and capital stock, especially where the investment is irreversible (Samaniego \& Sun, 2019). On the other hand, using a model in which even the depreciation amount itself is a fuzzy number under different scenarios, management can make more accurate investment decisions under uncertainty.

There are several studies in the literature claiming that the main basis for choosing the depreciation method is lowering the present value of tax payments (Albonico et al., 2014; Baumol, 1971; Berg \& Moore, 1989; Press \& Davidson, 1964). However, cash flow is not regular over the years. Even in times of global crisis, such as Covid-19, firms may not be able to obtain cash flow to cover depreciation. In an environment of such uncertainty, it is strategically important for management to make the right investment decisions. Unlike pre-existing studies, this study aims to help make investment decisions more accurate by using fuzzy numbers for the amount of future depreciation. More specifically, it proposes a model for evaluating investments not only in times when cash flows are uncertain but also in times when depreciation is uncertain. As mentioned before, several studies are using fuzzy numbers for cash flow (Berg et al., 2001; Berg \& Moore, 1989; Khalili et al., 2014; Samaniego \& Sun, 2019) but in these studies, the depreciation amount is fixed or predictable. However, this is unrealistic for periods when cash flow is completely interrupted (such as Covid-19). In this study, we developed a new model for making investment decisions in times when depreciation amount is uncertain, such as cash flow.

The remaining part of the study is organized as follows. In Section 2, we describe studies in the literature. Section 3, provides information about the depreciation method and the theoretical basis of depreciation. Section 4, we explained the methodology of the study, interval type- 2 fuzzy sets. The theory development process, and Application of interval type-2 fuzzy straight-line method of depreciation described in Sections 5 and Section 6. In Section 7, we conclude the finding of the study. 


\section{LITERATURE}

The first studies examining the relationship between depreciation policy and investment date back to the 1960s. Smith (1963), for example, examined the relationship between firms ' investment decisions and depreciation policy, taking into account factors such as the industry in which they operated, the method of depreciation, and tax advantage. Press and Davidson (1964) updated their article in 1961 due to legal changes. They suggested the "best" method of depreciation under existing laws. Besides this study, several studies are proposing the "best" or optimum method of depreciation (Baumol, 1971; Berg et al., 2001; Davidson \& Drake, 1961; De Waegenaere \& Wielhouwer, 2002; Stickney, 1981; Wakeman, 1980).

Companies often operate within an uncertain environment. Accordingly, their cash flows are uncertain. Several studies associate operating in an uncertain environment with the method of depreciation (Berg et al., 2001; Berg \& Moore, 1989; Femminis, 2008). By all means, in an environment of uncertainty, there is a consensus that there is an aggregate economic activity (Samaniego \& Sun, 2019). Accordingly, it seems that uncertainty significantly affects investment decisions, especially in high-depreciation industries, and it is covered in the literature about the depreciation method. Negative directional interaction between depreciation and uncertainty provides important evidence for this (Samaniego \& Sun, 2019).

Capital investment decisions maximize shareholder wealth by increasing the market value of firms. These decisions are also important for securing the long-term survival of firms (Jackson, 2008). However, decisions on capital investment are not independent of the depreciation method applied. In this context, there is evidence that there is a significant relationship between the different depreciation methods, the accelerated depreciation method, and the level of capital investments (Jackson et al., 2009). These findings also indicate that the depreciation method is remarkably effective in investment decisions.

Studies are examining various aspects of depreciation. For example, Jackson (2010) examined the relationship between the depreciation method and selling a used capital asset. In the study, decisionmakers considered accounting depreciation and historical cost when they sold a used capital asset. Kulp \& Hartman (2011) emphasized that the depreciation method was determined by taking into account the present value of expected tax payments and accelerated methods were preferred. In response to this situation, they developed a model which created conditions in which the straight-line depreciation model could be preferred. Yussof et al., (2014) analyzed the contrast between accounting depression and the tax treatment of capital allowance for Malaysia. According to the result of the study, it was proposed that the government redesign the current capital allowance system. Park (2016) investigated deprecation recovery periods based on the "bonus deprecation" method in the United States. According to the study's findings, the change in the depreciation method contributed positively to annual investment amounts. Rassenfosse \& Jaffe (2018) focused on estimating the depreciation rate with revenues associated with patent applications in Australia. They also analyzed the impact of patent protection on the depreciation rate. The findings showed that the impact of patent protection on the depreciation rate was $2-7 \%$. According to Samaniego \& Sun (2019), the number of investments decreases during periods of high uncertainty. This reduction is more evident, especially in industries where capital depreciation is rapid. The findings of their study show that growth is negatively affected by high uncertainty, which also affects depreciation. Ohrn (2019) examined the impact of depreciation policies on the manufacturing sector in the United States. According to the findings of the study, accelerated depreciation policies have an impact on capital investment.

Moreover, various studies in the literature examine the depreciation policy of advertising (Abdel-Khalik, 1975; Falk \& Miller, 1977; Hirschey \& Weygandt, 1985; Peles, 1971), goodwill amortization period (Hall, 1993; Henning \& Shaw, 2003; Jennings et al., 2001), determinants of goodwill and effects of goodwill amortization (Ayers et al., 2000; Duvall et al., 1992; Glaum et al., 2015; Huefner \& Largay, 2004; Vogt et al., 2016) and valuation of company and amortization (Gabriel, 1937; Lev \& Sougiannis, 1996). Although such articles examine depreciation in different dimensions, they don't contain information about the optimum method of depreciation for the firm under conditions of uncertainty. 


\section{INTERVAL TYPE-2 FUZZY SETS}

In the section, we describe interval type-2 fuzzy sets briefly. Type- 2 fuzzy sets were proposed by Zadeh as an extension of type-1 fuzzy sets having membership grades as type-1 fuzzy sets. A type- 2 fuzzy set $\tilde{A}$ in the universe of discourse $\mathrm{X}$ can be presented by a type-2 membership function $\mu_{\tilde{A}}$, viewed as shown in Eq. (1) (Zadeh, 1975; Mendel et al., 2006; Zeng et al., 2007):

$$
\tilde{A}=\left\{\left((x, u), \mu_{\tilde{\tilde{A}}}(x, u)\right) \mid \forall x \in X, \forall u \in J_{x} \subseteq[0,1], 0 \leq \mu_{\tilde{\tilde{A}}}(x, \mu) \leq 1\right\}
$$

Where $J_{x}$ states an interval [0,1]. The type-2 fuzzy set $\tilde{\tilde{A}}$ also can be represented as shown in Eq. (2) (Mendel et al., 2006):

$$
\tilde{\tilde{A}}=\int_{x \in X} \int_{u \in J_{X}} \mu_{\tilde{A}}(x, u) /(x, u)
$$

Where $J_{x} \subseteq[0,1]$ and $\int$ state union over all acceptable $x$ and $u$. Let $\tilde{\tilde{A}}$ be IT2FSs in the universe of discourse X presented by type-2 membership function $\mu_{\tilde{A}}$ If all, $\mu_{\tilde{A}}(x, u)=1$ after $\tilde{\tilde{A}}$ is called an IT2FSs (Zadeh, 1975; Buckley, 1985). An IT2FSs $\tilde{A}$ can pass for a particular situation of a type-2 fuzzy set, presented as shown in Eq. (3) and in Figure 1 and Figure 2 (Mendel et al, 2006).

$$
\tilde{A}=\int_{x \in X} \int_{u \in J_{X}} 1 /(x, u)
$$

\subsection{Triangular Interval Type-2 Fuzzy Sets}

A triangular IT2FS are defined as $\tilde{\tilde{A}}_{i}=\left(\left(a_{i l}^{U}, a_{i m}^{U}, a_{i u}^{U} ; H\left(A_{i}^{U}\right)\right),\left(a_{i l}^{L}, a_{i m}^{L}, a_{i u}^{L} ; H\left(\begin{array}{c}A_{i}^{L} \\ )\end{array}\right)\right)\right.$ where $A_{i}^{U}$ and $A_{i}{ }^{L}$ are type-1 fuzzy sets, $a_{i l}^{U}, a_{i m}^{U}, a_{i r}^{U}, a_{i l}^{L}, a_{i m}^{L}, a_{i r}^{L}$ are the references points of the IT2FSs $A_{i}$, $H\left(A_{i}^{U}\right)$ denotes the membership value of the element $a_{j(j+1)}^{U}$ in the upper triangular membership function $\left(\dot{A}_{i}^{U}\right), 1 \leq j \leq 2, H\left(\dot{A}_{i}^{L}\right)$ denotes the membership value of the element $a_{j(j+1)}^{L}$ in the lower triangular membership function $\left(\dot{\vdots}_{i}^{L}\right), 1 \leq j \leq 2, H\left(\dot{A}_{i}^{U}\right) \in[0,1], H\left(\dot{A}_{i}^{L}\right) \in[0,1]$, The membership function of a triangular interval type-2 fuzzy set is given in Figure 1.

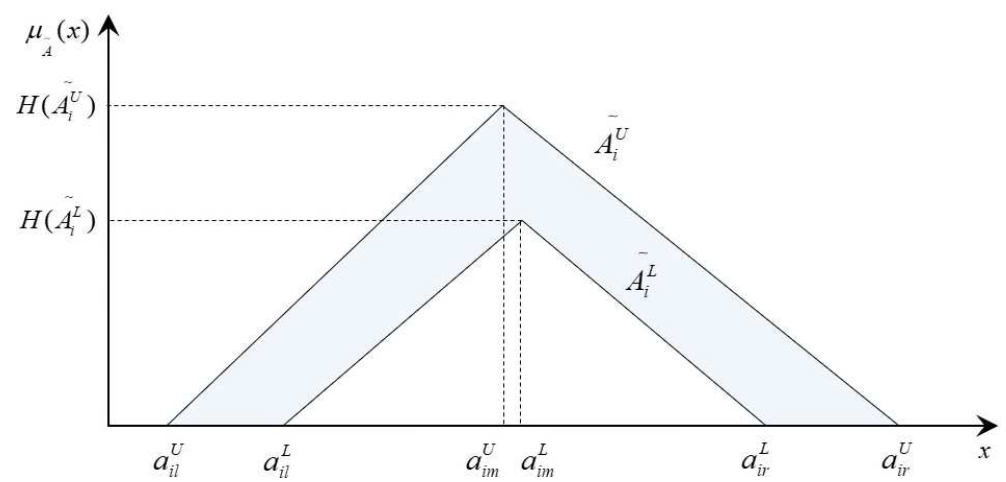

Figure 1. Triangular interval types 2 fuzzy numbers. 
The basic arithmetic operation of interval triangular type-2 fuzzy sets defined $\tilde{A}_{1}$ and $\tilde{A}_{2}$ are given as below, Eq. (4-11).

$$
\begin{aligned}
& \tilde{\tilde{A}}_{1}=\left(\left(a_{1 l}^{U}, a_{1 m}^{U}, a_{1 u}^{U} ; H\left(\dot{A}_{1}^{U}\right)\right),\left(a_{1 l}^{L}, a_{1 m}^{L}, a_{1 u}^{L} ; H\left(\dot{A}_{1}^{L}\right)\right)\right) \\
& \tilde{\tilde{A}}_{2}=\left(\left(a_{2 l}^{U}, a_{2 m}^{U}, a_{2 u}^{U} ; H\left(\dot{A}_{2}^{U}\right)\right),\left(a_{2 l}^{L}, a_{2 m}^{L}, a_{2 u}^{L} ; H\left(\begin{array}{c}
\vdots \\
A_{2}^{L}
\end{array}\right)\right)\right)
\end{aligned}
$$

Definition 1: The addition operation between the two triangular IT2FSs $\stackrel{\dot{A}}{1}$ and $\stackrel{\dot{A}}{2}$ is defined in $E q$. (4) as below.

$$
\begin{aligned}
\dot{A}_{1} \oplus \dot{A}_{2}= & \left(\left(a_{1 l}^{U}+a_{2 r}^{U}, a_{1 m}^{U}+a_{2 m}^{U}, a_{1 r}^{U}+a_{2 l}^{U} ; \min \left(H\left(\dot{A}_{1}^{U}\right) ; H\left(\dot{A}_{2}^{U}\right)\right)\right),\right. \\
& \left.\left(a_{1 l}^{L}+a_{2 r}^{L}, a_{1 m}^{L}+a_{2 m}^{L}, a_{1 r}^{L}+a_{2 l}^{L} ; \min \left(H\left(\dot{A}_{1}^{L}\right) ; H\left(\dot{A}_{2}^{L}\right)\right)\right)\right) .
\end{aligned}
$$

Definition 2: The subtraction operation between two the triangular IT2FSs $\stackrel{;}{A}$ and $\stackrel{;}{A_{2}}$ is defined in $E q$. (5) as below.

$$
\begin{aligned}
\dot{A}_{1} ! \dot{A}_{2}= & \left(\left(a_{1 l}^{U}-a_{2 r}^{U}, a_{1 m}^{U}-a_{2 m}^{U}, a_{1 r}^{U}-a_{2 l}^{U} ; \min \left(H\left(\dot{A}_{1}^{U}\right) ; H\left(\begin{array}{c}
\vdots \\
A_{2}^{U}
\end{array}\right)\right)\right),\right. \\
& \left.\left(a_{1 l}^{L}-a_{2 r}^{L}, a_{1 m}^{L}-a_{2 m}^{L}, a_{1 r}^{L}-a_{2 l}^{L} ; \min \left(H\left(\dot{A}_{1}^{L}\right) ; H\left(A_{2}^{L}\right)\right)\right)\right) .
\end{aligned}
$$

Definition 3: The multiplication operation between two the triangular IT2FSs $\stackrel{\dot{A}}{1}_{\text {and }} \dot{A}_{2}$ is defined in Eq. (6) as below.

$$
\begin{aligned}
\dot{A}_{1} \otimes \dot{A}_{2}= & \left(\left(a_{1 l}^{U} x a_{2 r}^{U}, a_{1 m}^{U} x a_{2 m}^{U}, a_{1 r}^{U} x a_{2 l}^{U} ; \min \left(H\left(\dot{A}_{1}^{U}\right) ; H\left(\dot{A}_{2}^{U}\right)\right)\right),\right. \\
& \left.\left(a_{1 l}^{L} x a_{2 r}^{L}, a_{1 m}^{L} x a_{2 m}^{L}, a_{1 r}^{L} x a_{2 l}^{L} ; \min \left(H\left(\dot{A}_{1}^{L}\right) ; H\left(\dot{A}_{2}^{L}\right)\right)\right)\right) .
\end{aligned}
$$

Definition 4: The arithmetic operation between the triangular IT2FSs $\dot{A}$ and a crisp value $k>0$ is defined in Eq. (7) and Eq. (8) as below.

$$
\begin{aligned}
& k \dot{A}_{1}=\left(\left(k \times a_{1 l}^{U}, k \times a_{1 m}^{U}, k \times a_{1 r}^{U} ; H\left(A_{1}^{U}\right)\right),\left(k \times a_{1 l}^{L}, k \times a_{1 m}^{L}, k \times a_{1 r}^{L} ; H\left(A_{1}^{L}\right)\right)\right) . \\
& \frac{\dot{A}_{1}}{k}=\left(\left(\frac{1}{k} \times a_{1 l}^{U}, \frac{1}{k} \times a_{1 m}^{U}, \frac{1}{k} \times a_{1 r}^{U} ; H\left(\dot{A}_{1}^{U}\right)\right),\left(\frac{1}{k} \times a_{1 l}^{L}, \frac{1}{k} \times a_{1 m}^{L}, \frac{1}{k} \times a_{1 r}^{L} ; H\left(A_{1}^{L}\right)\right)\right) .
\end{aligned}
$$

Definition 5: The division operation for two the triangular IT2FSs $\stackrel{\dot{A}}{1}_{1}$ and $\dot{A}_{2}$ is defined in Eq. (9) as below. 


$$
\begin{array}{r}
\frac{\dot{A_{1}}}{\dot{A_{2}} \cong}\left(\left(\frac{a_{1 l}^{U}}{a_{2 r}^{U}}, \frac{a_{1 m}^{U}}{a_{2 m}^{U}}, \frac{a_{1 r}^{U}}{a_{2 l}^{U}} ; \min \left(H\left(A_{1}^{U}\right) ; H\left(\dot{A}_{2}^{U}\right)\right)\right),\right. \\
\left.\left(\frac{a_{1 l}^{L}}{a_{2 r}^{L}}, \frac{a_{1 m}^{L}}{a_{2 m}^{L}}, \frac{a_{1 r}^{L}}{a_{2 l}^{L}} ; \min \left(H\left(\begin{array}{c}
\vdots \\
A_{1}^{U}
\end{array}\right) ; H\left(\begin{array}{c}
\vdots \\
A_{2}^{U}
\end{array}\right)\right)\right)\right) .
\end{array}
$$

Definition 6: The inverse operation of the triangular IT2FSs $\stackrel{;}{A}_{1}$ is defined in Eq. (10) as below.

$$
\frac{1}{\dot{A}_{1}}=\left(\left(\frac{1}{a_{1 r}^{U}}, \frac{1}{a_{1 m}^{U}}, \frac{1}{a_{1 l}^{U}} ; H\left(A_{1}^{U}\right)\right),\left(\frac{1}{a_{1 r}^{L}}, \frac{1}{a_{1 m}^{L}}, \frac{1}{a_{1 l}^{L}} ; H\left(A_{1}^{L}\right)\right)\right) .
$$

Definition 7: $n^{\text {th }}$ root operation of the triangular IT2FSs $\dot{A}_{1}$ is defined in Eq. (11) as below.

$$
\sqrt[n]{\dot{A}_{1}}=\left(\left(\sqrt[n]{a_{1 l}^{U}}, \sqrt[n]{a_{1 m}^{U}}, \sqrt[n]{a_{1 r}^{U}} ; H\left(A_{1}^{U}\right)\right),\left(\sqrt[n]{a_{1 l}^{L}}, \sqrt[n]{a_{1 m}^{L}}, \sqrt[n]{a_{1 r}^{L}} ; H\left(\dot{A}_{1}^{L}\right)\right)\right)
$$

\subsection{Trapezoidal Interval Type-2 Fuzzy Sets}

A trapezoidal interval type-2 fuzzy sets are defined as $\tilde{A}=\left(\left(a_{i 1}^{U}, a_{i 2}^{U}, a_{i 3}^{U}, a_{i 4}^{U} ; H_{1}\left(\dot{\vdots} A_{i}^{U}\right), H_{2}\left(\dot{\vdots} A_{i}^{U}\right)\right)\right.$, $\left.\left(a_{i 1}^{L}, a_{i 2}^{L}, a_{i 3}^{L}, a_{i 4}^{L} ; H_{1}\left(\dot{A}_{i}^{L}\right), H_{2}\left(\dot{A}_{i}^{L}\right)\right)\right)$ where $\dot{A}_{i}^{U} \quad$ and $A_{i}^{L}$ are type-1 fuzzy sets, $a_{i 1}^{U}, a_{i 2}^{U}, a_{i 3}^{U}$, $a_{i 4}^{U}, a_{i 1}^{L}, a_{i 2}^{L}, a_{i 3}^{L}, a_{i 4}^{L}$ are the references points of the interval trapezoidal type-2 fuzzy sets $\dot{A}_{i}, H_{j}\left(\dot{A}_{i}^{U}\right)$; states the membership estimation of the factor $a_{j(j+1)}^{U}$ in the upper trapezoidal membership function $\left(\begin{array}{c}\vdots \\ A_{i}^{U}\end{array}\right), 1 \leq j \leq 2, H_{j}\left(A_{i}^{L}\right)$; also it states the membership estimation of the factor $a_{j(j+1)}^{L}$ in the lower trapezoidal membership function $\left(\vdots A_{i}^{L}\right), 1 \leq j \leq 2, \quad H_{1}\left(\dot{A}_{i}^{U}\right) \in[0,1], \quad H_{2}\left(\dot{A}_{i}^{U}\right) \in[0,1], H_{1}\left(\dot{A}_{i}^{L}\right) \in[0,1]$, $H_{2}\left(\dot{A}_{i}^{L}\right) \in[0,1]$ and $1 \leq j \leq n$ (Chen and Lee, 2010). The membership function of a trapezoidal interval type-2 fuzzy set is given in Figure 2.

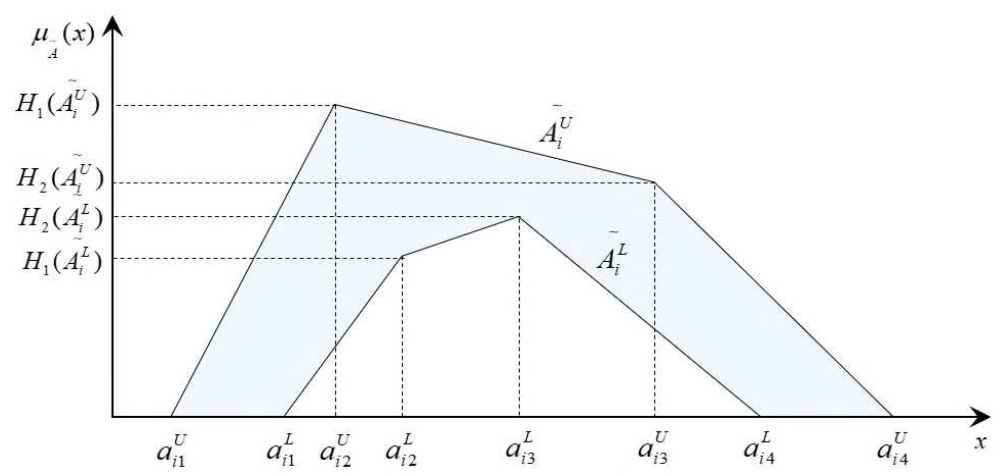

Figure 2. Trapezoidal interval types 2 fuzzy numbers. 
The basic arithmetic operation of interval trapezoidal type-2 fuzzy sets defined $\tilde{A}_{1}$ and $\tilde{\tilde{A}}_{2}$ are given as below, Eq. (12-19).

$$
\begin{aligned}
& \tilde{A}_{1}=\left(\left(a_{11}^{U}, a_{12}^{U}, a_{13}^{U}, a_{14}^{U} ; H_{1}\left(\vdots A_{1}^{U}\right), H_{2}\left(\dot{A}_{1}^{U}\right)\right),\left(a_{11}^{L}, a_{12}^{L}, a_{13}^{L}, a_{14}^{L} ; H_{1}\left(\dot{A}_{1}^{L}\right), H_{2}\left(\dot{A}_{1}^{L}\right)\right)\right) \\
& \tilde{A}_{2}=\left(\left(a_{21}^{U}, a_{22}^{U}, a_{23}^{U}, a_{24}^{U} ; H_{1}\left(\dot{\vdots} A_{2}^{U}\right), H_{2}\left(\dot{\vdots} A_{2}^{U}\right)\right),\left(a_{21}^{L}, a_{22}^{L}, a_{23}^{L}, a_{24}^{L} ; H_{1}\left(\dot{A}_{2}^{L}\right), H_{2}\left(\dot{\vdots} \dot{A}_{2}^{L}\right)\right)\right)
\end{aligned}
$$

Definition 1: The addition operation for the two trapezoidal IT2FSs $\stackrel{\dot{A}}{1}$ and $\stackrel{\dot{A}}{2}$ is defined in Eq. (12) as below.

$$
\begin{aligned}
& \dot{A}_{1} \oplus \dot{\bar{A}}_{2}=\left(\left(a_{11}^{U}+a_{21}^{U}, a_{12}^{U}+a_{22}^{U}, a_{13}^{U}+a_{23}^{U}, a_{14}^{U}+a_{24}^{U} ;\right.\right. \\
& \left.\min \left(H_{1}\left(\begin{array}{c}
\vdots \\
A_{1}^{U}
\end{array}\right) ; H_{1}\left(\begin{array}{c}
\vdots \\
A_{2}^{U}
\end{array}\right)\right), \min \left(H_{2}\left(\begin{array}{c}
\vdots \\
A_{1}^{U}
\end{array}\right) ; H_{2}\left(\begin{array}{c}
\vdots \\
A_{2}^{U}
\end{array}\right)\right)\right), \\
& \left(a_{11}^{L}+a_{21}^{L}, a_{12}^{L}+a_{22}^{L}, a_{13}^{L}+a_{23}^{L}, a_{14}^{L}+a_{24}^{L} ;\right. \\
& \left.\min \left(H_{1}\left(\dot{A}_{1}^{L}\right) ; H_{1}\left(\dot{A}_{2}^{L}\right)\right), \min \left(H_{2}\left(\dot{A}_{1}^{L}\right) ; H_{2}\left(\dot{A}_{2}^{L}\right)\right)\right) \text {. }
\end{aligned}
$$

Definition 2: The subtraction operation for two the trapezoidal IT2FSs $\stackrel{;}{A_{1}}$ and $\dot{\dot{A}}$ is defined in Eq. (13) as below.

$$
\begin{aligned}
& \dot{A}_{1} ! \quad \dot{A}_{2}=\left(\left(a_{11}^{U}-a_{24}^{U}, a_{12}^{U}-a_{23}^{U}, a_{13}^{U}-a_{22}^{U}, a_{14}^{U}-a_{21}^{U} ;\right.\right. \\
& \left.\min \left(H_{1}\left(\begin{array}{c}
\vdots \\
A_{1}^{U}
\end{array}\right) ; H_{1}\left(\begin{array}{c}
\vdots \\
A_{2}^{U}
\end{array}\right)\right), \min \left(H_{2}\left(\begin{array}{c}
\vdots \\
A_{1}^{U}
\end{array}\right) ; H_{2}\left(\begin{array}{c}
\vdots \\
A_{2}^{U}
\end{array}\right)\right)\right), \\
& \left(a_{11}^{L}-a_{24}^{L}, a_{12}^{L}-a_{23}^{L}, a_{13}^{L}-a_{22}^{L}, a_{14}^{L}-a_{21}^{L}\right. \text {; } \\
& \left.\min \left(H_{1}\left(\dot{A}_{1}^{L}\right) ; H_{1}\left(\dot{A}_{2}^{L}\right)\right), \min \left(H_{2}\left(\dot{A}_{1}^{L}\right) ; H_{2}\left(\dot{A}_{2}^{L}\right)\right)\right) \text {. }
\end{aligned}
$$

Definition 3: The multiplication operation for two the trapezoidal IT2FSs $\dot{A}_{1}$ and $\dot{\leftrightarrow}_{2}$ is defined in Eq. (14) as below.

$$
\begin{aligned}
& \dot{A}_{1} \otimes \dot{A}_{2}=\left(\left(a_{11}^{U} x a_{21}^{U}, a_{12}^{U} x a_{22}^{U}, a_{13}^{U} x a_{23}^{U}, a_{14}^{U} x a_{24}^{U} ;\right.\right. \\
& \left.\min \left(H_{1}\left(\begin{array}{c}
\vdots \\
A_{1}^{U}
\end{array}\right) ; H_{1}\left(\begin{array}{c}
\vdots \\
A_{2}^{U}
\end{array}\right)\right), \min \left(H_{2}\left(\dot{A}_{1}^{U}\right) ; H_{2}\left(\begin{array}{c}
\vdots \\
A_{2}^{U}
\end{array}\right)\right)\right), \\
& \left(a_{11}^{L} x a_{21}^{L}, a_{12}^{L} x a_{22}^{L}, a_{13}^{L} x a_{23}^{L}, a_{14}^{L} x a_{24}^{L}\right. \text {; } \\
& \left.\min \left(H_{1}\left(\begin{array}{c}
\vdots \\
A_{1}^{L}
\end{array}\right) ; H_{1}\left(\dot{A}_{2}^{L}\right)\right), \min \left(H_{2}\left(\dot{A}_{1}^{L}\right) ; H_{2}\left(\dot{A}_{2}^{L}\right)\right)\right) \text {. }
\end{aligned}
$$

Definition 4: The arithmetic operation for the trapezoidal IT2FSs $\stackrel{A}{1}_{1}$ and a crisp value $k>0$ is defined in Eq. (15) and Eq. (16) as below. 


$$
\begin{aligned}
& k \dot{A_{1}}=\left(\left(k \times a_{11}^{U}, k \times a_{12}^{U}, k \times a_{13}^{U}, k \times a_{14}^{U} ; H_{1}\left(\dot{\vdots} A_{1}^{U}\right), H_{2}\left(\dot{\vdots} A_{1}^{U}\right)\right),\right. \\
& \left.\left(k \times a_{11}^{L}, k \times a_{12}^{L}, k \times a_{13}^{L}, k \times a_{14}^{L} ; H_{1}\left(\begin{array}{c}
A_{1}^{L} \\
)
\end{array}\right) H_{2}\left(\dot{A}_{1}^{L}\right)\right)\right) . \\
& \frac{\dot{\dot{A}}}{k}=\left(\left(\frac{1}{k} \times a_{11}^{U}, \frac{1}{k} \times a_{12}^{U}, \frac{1}{k} \times a_{13}^{U}, \frac{1}{k} \times a_{14}^{U} ; H_{1}\left(\dot{A}_{1}^{U}\right), H_{2}\left(\dot{\vdots} A_{1}^{U}\right)\right.\right. \text {, } \\
& \left(\frac{1}{k} \times a_{11}^{L}, \frac{1}{k} \times a_{12}^{L}, \frac{1}{k} \times a_{13}^{L}, \frac{1}{k} \times a_{14}^{L} ; H_{1}\left(\dot{A}_{1}^{L}\right), H_{2}\left(\dot{\vdots} A_{1}^{L}\right)\right) .
\end{aligned}
$$

Definition 5: The division operation for two the trapezoidal IT2FSs $\stackrel{\dot{A}}{1}_{1}$ and $\stackrel{\dot{A}}{2}_{2}$ is defined in Eq. (17) as below.

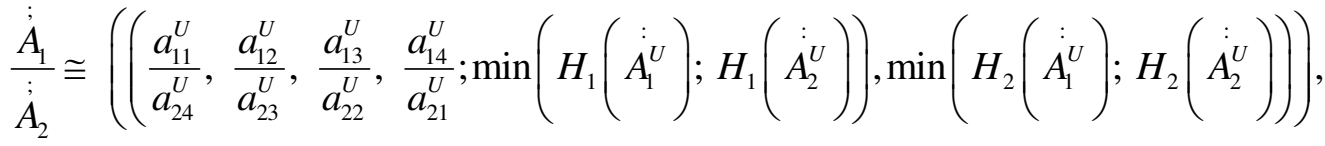

$$
\begin{aligned}
& \left.\left(\frac{a_{11}^{L}}{a_{24}^{L}}, \frac{a_{12}^{L}}{a_{23}^{L}}, \frac{a_{13}^{L}}{a_{22}^{L}}, \frac{a_{14}^{L}}{a_{21}^{L}} ; \min \left(H_{1}\left(\dot{A}_{1}^{L}\right) ; H_{1}\left(\dot{A}_{2}^{L}\right)\right), \min \left(H_{2}\left(\vdots A_{1}^{L}\right) ; H_{2}\left(\vdots A_{2}^{L}\right)\right)\right)\right) \text {. }
\end{aligned}
$$

Definition 6: The inverse operation for the trapezoidal IT2FSs $\dot{A}_{1}$ is defined in Eq. (18) as below.

$$
\begin{array}{r}
\frac{1}{\dot{A}_{1}}=\left(\left(\frac{1}{a_{14}^{U}}, \frac{1}{a_{13}^{U}}, \frac{1}{a_{12}^{U}}, \frac{1}{a_{11}^{U}} ; H_{1}\left(\dot{A}_{1}^{U}\right) ; H_{1}\left(\dot{A}_{2}^{U}\right)\right),\right. \\
\left.\left(\frac{1}{a_{14}^{L}}, \frac{1}{a_{13}^{L}}, \frac{1}{a_{12}^{L}}, \frac{1}{a_{11}^{L}} ; H_{1}\left(\dot{A}_{1}^{L}\right) ; H_{1}\left(\dot{A}_{2}^{L}\right)\right)\right) .
\end{array}
$$

Definition 7: $n^{\text {th }}$ root operation for the trapezoidal IT2FSs $\dot{A}_{1}$ is defined in Eq. (19) as below.

$$
\begin{aligned}
\sqrt[n]{\dot{\leftrightarrow}_{1}}= & \left(\left(\sqrt[n]{a_{11}^{U}}, \sqrt[n]{a_{12}^{U}}, \sqrt[n]{a_{13}^{U}}, \sqrt[n]{a_{14}^{U}} ; H_{1}\left(\dot{A}_{1}^{U}\right) ; H_{1}\left(A_{2}^{U}\right)\right),\right. \\
& \left.\left(\sqrt[n]{a_{11}^{L}}, \sqrt[n]{a_{12}^{L}}, \sqrt[n]{a_{13}^{L}}, \sqrt[n]{a_{14}^{L}} ; H_{1}\left(A_{1}^{L}\right) ; H_{1}\left(\dot{A}_{2}^{L}\right)\right)\right) .
\end{aligned}
$$

\subsection{Interval Type-2 Fuzzy Defuzzification}

The method of criteria Center of Area (COA) is considered to defuzzify the lower and upper membership values of IT2FSs into Best Nonfuzzy Performance (BNP) value in the study. The BNP value is worked out in Eq. (20) and Eq. (21) as below (Bellman \& Zadeh, 1970; Opricovic \& Tzeng, 2003; Hsieh et al., 2004). Accordingly, it is calculated by applying arithmetic mean for each defuzzification value of $A_{i}^{U}$ and $A_{i}^{L}$.

$$
\begin{aligned}
& \dot{w}_{j}=\left(w_{j 3} w_{j 4}-w_{j 1} w_{j 2}+\frac{\left(w_{j 4}-w_{j 3}\right)^{2}-\left(w_{j 2}-w_{j 1}\right)^{2}}{3}\right) /\left(w_{j 3}+w_{j 4}-w_{j 1}-w_{j 2}\right) \\
& B N P_{i}=l_{i}+\left[\frac{\left(u_{i}-l_{i}\right)+\left(m_{i}-l_{i}\right)}{3}\right], \forall i
\end{aligned}
$$




\section{INTERVAL TYPE-2 FUZZY DEPRECIATION}

In this section, Straight-Line Depreciation and Double Declining Balance (Accelerated) methods are described in terms of interval type-2 fuzzy sets.

\subsection{Straight-Line Depreciation Method}

The straight-line depreciation method allocates an equal portion of the depreciable value in each period of the asset's useful life. The assumption of the method is the depreciation is a function of the passage of time rather than the actual productive use of the asset. The depreciation expense for a period is equally calculated by dividing the depreciable cost of the asset by the years of the asset's useful life. In the method, the depreciable cost is calculated by deducting salvage value from the cost of the asset. Depreciable cost is arrived at by deducting salvage or residual value from the original cost of the asset. So, the value of the asset equals to salvage value when the end of the useful life.

The equation is used for calculating depreciation under the classic straight-line method of depreciation. By modifying the Eq. (22) for ship's deprecation for maritime. The Equation has been described as below in Eq. (22-23):

$$
\begin{aligned}
D_{\text {deprecation }} & =\frac{1}{L_{\text {life }}}\left(C_{\text {cost }}-S_{\text {salvage }}\right) \\
& =\frac{1}{L_{\text {life }}}\left(C_{\cos t}-L D T_{\text {displacement }} P_{\text {price }}\right) \\
S_{\text {salvage }}= & L D T_{\text {displacement }} P_{\text {price }}
\end{aligned}
$$

where;

$D \quad$ Depreciation

$C \quad$ Cost (Ship's Price)

$S \quad$ Salvage Value

$L \quad$ Useful Life $\forall L \in R$ and $L>0$

LDT Light Ship Displacement

$P \quad$ Price of scrap metal

Interval type-2 fuzzy straight-line depreciation method is defined in Eq. (24). Also, Interval type-2 fuzzy salvage for a ship is defined in Eq. (25).

$$
\begin{aligned}
& \dot{\dot{D}}_{\text {deprecation }}=\frac{1}{L_{\text {life }}}\left(\dot{⿱}_{\text {cost }}-\dot{;}_{\text {salvage }}\right)
\end{aligned}
$$

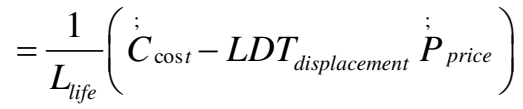

$$
\begin{aligned}
& \dot{S}_{\text {salvage }}=L D T_{\text {displacement }} \stackrel{\dot{P}}{\text { price }}
\end{aligned}
$$

where;

$$
\begin{array}{cl}
\dot{;} & \text { interval type-2 fuzzy Depreciation } \\
\dot{C} & \text { interval type-2 fuzzy Cost (Ship's Price) } \\
\dot{S} & \text { interval type-2 fuzzy Salvage Value } \\
L & \text { Useful Life } \forall L \in R \text { and } L>0 \\
L D T & \text { Light Ship Displacement } \\
\dot{P} & \text { interval type-2 fuzzy Price of scrap metal }
\end{array}
$$




\subsubsection{Triangular Interval Type-2 Fuzzy Straight-Line Depreciation Method}

Triangular Interval Type-2 fuzzy straight-line depreciation method as variable of $\dot{D}_{i}, \dot{C}_{i}, P_{i}$ and $S_{i}$ are occurred at the time $i$ as follows and $\stackrel{\dot{D}}{D_{i}}$ is defined in Eq. (26):

$$
\begin{aligned}
\tilde{\tilde{D}}_{i}= & \left(\left(d_{i l}^{U}, d_{i m}^{U}, d_{i r}^{U} ; H_{1}\left(\tilde{d_{i}^{U}}\right)\right),\left(d_{i l}^{L}, d_{i m}^{L}, d_{i r}^{L} ; H_{1}\left(\tilde{d}_{i}^{L}\right)\right)\right) \\
= & \frac{1}{L_{\text {life }}}\left[\left(\left(c_{i l}^{U}, c_{i m}^{U}, c_{i r}^{U} ; H_{1}\left(\tilde{c_{i}^{U}}\right)\right),\left(c_{i l}^{L}, c_{i m}^{L}, c_{i r}^{L} ; H_{1}\left(\tilde{c_{i}^{L}}\right)\right)\right)-\right. \\
& \left.L D T_{\text {displacement }}\left(\left(p_{i l}^{U}, p_{i m}^{U}, p_{i r}^{U} ; H_{1}\left(\tilde{p_{i}^{U}}\right)\right),\left(p_{i l}^{L}, p_{i m}^{L}, p_{i r}^{L} ; H_{1}\left(\tilde{p_{i}^{L}}\right)\right)\right)\right]
\end{aligned}
$$

$\dot{\sim}_{i}, \stackrel{\dot{P}}{P_{i}}$ and $\stackrel{\dot{S}}{S}_{i}$ are defined for triangular IT2FSs as follows in Eq. (27-29):

$$
\begin{aligned}
\tilde{\tilde{C}}_{i} & =\left(\left(c_{i l}^{U}, c_{i m}^{U}, c_{i r}^{U} ; H_{1}\left(\tilde{c_{i}^{U}}\right)\right),\left(c_{i l}^{L}, c_{i m}^{L}, c_{i r}^{L} ; H_{1}\left(\tilde{c_{i}^{L}}\right)\right)\right) \\
\tilde{P}_{i} & =\left(\left(p_{i l}^{U}, p_{i m}^{U}, p_{i r}^{U} ; H_{1}\left(\tilde{p_{i}^{U}}\right)\right),\left(p_{i l}^{L}, p_{i m}^{L}, p_{i r}^{L} ; H_{1}\left(\tilde{p_{i}^{L}}\right)\right)\right) \\
\tilde{S}_{i} & =\left(\left(s_{i l}^{U}, s_{i m}^{U}, s_{i r}^{U} ; H_{1}\left(\tilde{s_{i}^{U}}\right)\right),\left(s_{i l}^{L}, s_{i m}^{L}, s_{i r}^{L} ; H_{1}\left(\tilde{s_{i}^{L}}\right)\right)\right) \\
& =L D T_{\text {displacement }}\left(\left(p_{i l}^{U}, p_{i m}^{U}, p_{i r}^{U} ; H_{1}\left(\tilde{p_{i}^{U}}\right)\right),\left(\left(p_{i l}^{L}, p_{i m}^{L}, p_{i r}^{L} ; H_{1}\left(\tilde{p_{i}^{L}}\right)\right)\right)\right)
\end{aligned}
$$

\subsubsection{Trapezoidal Interval Type-2 Fuzzy Straight-Line Depreciation Method}

Trapezoidal Interval Type-2 fuzzy straight-line depreciation method as variable of $\dot{D}_{i}, \dot{C}_{i}, \dot{P}_{i}$ and $\dot{S}_{i}$ are occurred at the time $i$ as follows and $\stackrel{\dot{D}}{D_{i}}$ is defined in Eq. (30):

$$
\begin{aligned}
\tilde{\tilde{D}}_{i}= & \left(\left(d_{i 1}^{U}, d_{i 2}^{U}, d_{i 3}^{U}, d_{i 4}^{U} ; H_{1}\left(\tilde{d_{i}^{U}}\right), H_{1}\left(\tilde{d_{i}^{U}}\right)\right),\left(d_{i 1}^{L}, d_{i 2}^{L}, d_{i 3}^{L}, d_{i 4}^{L} ; H_{1}\left(\tilde{d_{i}^{L}}\right), H_{1}\left(\tilde{d_{i}^{L}}\right)\right)\right) \\
= & \frac{1}{L_{\text {life }}}\left[\left(\left(c_{i 1}^{U}, c_{i 2}^{U}, c_{i 3}^{U}, c_{i 4}^{U} ; H_{1}\left(\tilde{c_{i}^{U}}\right), H_{1}\left(\tilde{c_{i}^{U}}\right)\right),\left(c_{i 1}^{L}, c_{i 2}^{L}, c_{i 3}^{L}, c_{i 4}^{L} ; H_{1}\left(\tilde{c_{i}^{L}}\right), H_{1}\left(\tilde{c_{i}^{L}}\right)\right)\right)-\right. \\
& L D T_{\text {displacement }}\left(\left(p_{i 1}^{U}, p_{i 2}^{U}, p_{i 3}^{U}, p_{i 4}^{U} ; H_{1}\left(\tilde{p_{i}^{U}}\right), H_{1}\left(\tilde{p_{i}^{U}}\right)\right),\right. \\
& \left.\left.\left(p_{i 1}^{L}, p_{i 2}^{L}, p_{i 3}^{L}, p_{i 4}^{L} ; H_{1}\left(\tilde{p_{i}^{L}}\right), H_{1}\left(\tilde{p_{i}^{L}}\right)\right)\right)\right]
\end{aligned}
$$

$\dot{C}_{i}, \dot{P}_{i}$ and $\dot{S}_{i}$ are defined for trapezoidal IT2FSs as follows in Eq. (31-33):

$$
\stackrel{\vec{C}}{i}_{i}=\left(\left(c_{i 1}^{U}, c_{i 2}^{U}, c_{i 3}^{U}, c_{i 4}^{U} ; H_{1}\left(\tilde{c_{i}^{U}}\right), H_{1}\left(\tilde{c_{i}^{U}}\right)\right),\left(c_{i 1}^{L}, c_{i 2}^{L}, c_{i 3}^{L}, c_{i 4}^{L} ; H_{1}\left(\tilde{c_{i}^{L}}\right), H_{1}\left(\tilde{c_{i}^{L}}\right)\right)\right)
$$




$$
\begin{aligned}
\stackrel{m}{P}_{i}= & \left(\left(p_{i 1}^{U}, p_{i 2}^{U}, p_{i 3}^{U}, p_{i 4}^{U} ; H_{1}\left(\begin{array}{c}
\vdots \\
p_{i}^{U}
\end{array}\right), H_{1}\left(\begin{array}{c}
\vdots \\
p_{i}^{U}
\end{array}\right)\right),\left(p_{i 1}^{L}, p_{i 2}^{L}, p_{i 3}^{L}, p_{i 4}^{L} ; H_{1}\left(\begin{array}{c}
\vdots \\
p_{i}^{L}
\end{array}\right), H_{1}\left(\begin{array}{c}
: \\
p_{i}^{L}
\end{array}\right)\right)\right) \\
\stackrel{S}{S}_{i}= & \left(\left(s_{i 1}^{U}, s_{i 2}^{U}, s_{i 3}^{U}, s_{i 4}^{U} ; H_{1}\left(\begin{array}{c}
\vdots \\
s_{i}^{U}
\end{array}\right), H_{1}\left(\begin{array}{c}
\vdots \\
s_{i}^{U}
\end{array}\right)\right),\left(s_{i 1}^{L}, s_{i 2}^{L}, s_{i 3}^{L}, s_{i 4}^{L} ; H_{1}\left(\begin{array}{c}
\vdots \\
s_{i}^{L}
\end{array}\right), H_{1}\left(\begin{array}{c}
\vdots \\
s_{i}^{L}
\end{array}\right)\right)\right) \\
= & L D T_{\text {displacement }}\left(\left(p_{i 1}^{U}, p_{i 2}^{U}, p_{i 3}^{U}, p_{i 4}^{U} ; H_{1}\left(\begin{array}{c}
\vdots \\
p_{i}^{U}
\end{array}\right), H_{1}\left(\begin{array}{c}
\vdots \\
p_{i}^{U}
\end{array}\right)\right),\right. \\
& \left.\left(\left(p_{i 1}^{L}, p_{i 2}^{L}, p_{i 3}^{L}, p_{i 4}^{L} ; H_{1}\left(\begin{array}{c}
\vdots \\
p_{i}^{L}
\end{array}\right), H_{1}\left(\begin{array}{c}
\vdots \\
p_{i}^{L}
\end{array}\right)\right)\right)\right)
\end{aligned}
$$

\subsection{Double Declining Balance Depreciation Method}

The method of double-declining balance depreciation is a type of accelerated depreciation doubling the standard depreciation method. It is generally used to depreciate fixed assets more intensely in the early years, allowing the organization to postpone income taxes to the following years. The depreciation factor of the double-declining-balance method is the double value of the straight-line expense method. The method of double-declining-balance depreciation presents in a larger amount expensed in the earlier years, on the contrary, the later years of an asset's useful life. So, the value of the asset equals to salvage value when the end of the useful life.

The equation is used for calculating depreciation under the classic double-declining balance depreciation method. By modifying the Eq. (34) for ship's deprecation for maritime. The Equation has been described as below in Eq. (34-36):

$$
\begin{aligned}
& D_{\text {deprecation }}=R_{\text {rate }} x C_{\text {cost }} \\
& R_{\text {rate }}=\left(\frac{100 \%}{L_{\text {life }}}\right) \times 2 \\
& S_{\text {salvage }}=L D T_{\text {displacement }} P_{\text {price }}
\end{aligned}
$$

where:

$D \quad$ Depreciation

C Cost (Ship's Price) (Book Value at Beginning)

$S \quad$ Salvage Value

$L \quad$ Useful Life $\forall L \in R$ and $L>0$

LDT Light Ship Displacement

$P \quad$ Price of scrap metal

$R \quad$ Double Declining Balance Depreciation Rate

Interval type-2 fuzzy double-declining balance depreciation method is defined in Eq. (37). Also, a double-declining balance depreciation rate is defined in Eq. (38) and Interval type-2 fuzzy salvage for a ship is defined in Eq. (39).

$$
\begin{aligned}
& \dot{\dot{D}}_{\text {deprecation }}=R_{\text {rate }} x \dot{C}_{\cos t} \\
& R_{\text {rate }}=\left(\frac{100 \%}{L_{\text {life }}}\right) x 2 \\
& \dot{S}_{\text {salvage }}=L D T_{\text {displacement }} P_{\text {price }}
\end{aligned}
$$

where;

$D$ interval type-2 fuzzy depreciation 

C interval type-2 fuzzy Cost (Ship's Price)
$S \quad$ interval type-2 fuzzy Salvage Value
$L \quad$ Useful Life $\forall L \in R$ and $L>0$
LDT Light Ship Displacement
$\begin{array}{ll}P & \text { interval type-2 fuzzy Price of Scrap metal } \\ R & \text { Double Declining Balance Depreciation Rate }\end{array}$

\subsubsection{Triangular Interval Type-2 Fuzzy Double Declining Balance Depreciation Method}

Triangular Interval Type-2 fuzzy straight-line depreciation method as variable of $\dot{D}_{i}, \dot{C}_{i}, \dot{i}_{i}$ and $\dot{\dot{S}}_{i}$ are occurring at the time $i$ as follows and $D_{i}$ is defined in Eq. (40):

$$
\begin{aligned}
\tilde{\tilde{D}}_{i} & =\left(\left(d_{i l}^{U}, d_{i m}^{U}, d_{i r}^{U} ; H_{1}\left(\tilde{d_{i}^{U}}\right)\right),\left(d_{i l}^{L}, d_{i m}^{L}, d_{i r}^{L} ; H_{1}\left(\tilde{d_{i}^{L}}\right)\right)\right) \\
& =R_{\text {rate }} x\left(\left(c_{i l}^{U}, c_{i m}^{U}, c_{i r}^{U} ; H_{1}\left(\tilde{c_{i}^{U}}\right)\right),\left(c_{i l}^{L}, c_{i m}^{L}, c_{i r}^{L} ; H_{1}\left(\tilde{c_{i}^{L}}\right)\right)\right)
\end{aligned}
$$

$\dot{C}_{i}, \dot{i}_{i}$ and $\dot{S}_{i}$ are defined for triangular IT2FSs as follows in Eq. $\left(41-43 \dot{C}_{i}, \dot{\dot{P}}_{i}\right.$ and $\dot{\dot{S}}_{i}$ are defined for triangular IT2FSs as follows in Eq. (27-29):

$$
\begin{aligned}
\tilde{\tilde{C}}_{i} & =\left(\left(c_{i l}^{U}, c_{i m}^{U}, c_{i r}^{U} ; H_{1}\left(\tilde{c_{i}^{U}}\right)\right),\left(c_{i l}^{L}, c_{i m}^{L}, c_{i r}^{L} ; H_{1}\left(\tilde{c_{i}^{L}}\right)\right)\right) \\
\tilde{\tilde{P}}_{i} & =\left(\left(p_{i l}^{U}, p_{i m}^{U}, p_{i r}^{U} ; H_{1}\left(\tilde{p_{i}^{U}}\right)\right),\left(p_{i l}^{L}, p_{i m}^{L}, p_{i r}^{L} ; H_{1}\left(\tilde{p_{i}^{L}}\right)\right)\right) \\
\tilde{S}_{i} & =\left(\left(s_{i l}^{U}, s_{i m}^{U}, s_{i r}^{U} ; H_{1}\left(\tilde{s_{i}^{U}}\right)\right),\left(s_{i l}^{L}, s_{i m}^{L}, s_{i r}^{L} ; H_{1}\left(\tilde{s_{i}^{L}}\right)\right)\right) \\
& =L D T_{\text {displacement }}\left(\left(p_{i l}^{U}, p_{i m}^{U}, p_{i r}^{U} ; H_{1}\left(\tilde{p_{i}^{U}}\right)\right),\left(\left(p_{i l}^{L}, p_{i m}^{L}, p_{i r}^{L} ; H_{1}\left(\tilde{p_{i}^{L}}\right)\right)\right)\right)
\end{aligned}
$$

\subsubsection{Trapezoidal Interval Type-2 Fuzzy Double Declining Balance Depreciation Method}

Trapezoidal Interval Type-2 fuzzy straight-line depreciation method as variable of $\dot{D}_{i}, \dot{C}_{i}, \dot{P}_{i}$ and $\dot{S}_{i}$ are occurring at the time $i$ as follows and $\dot{D}_{i}$ is defined in Eq. (44):

$$
\begin{aligned}
\tilde{\tilde{D}}_{i} & =\left(\left(d_{i 1}^{U}, d_{i 2}^{U}, d_{i 3}^{U}, d_{i 4}^{U} ; H_{1}\left(\tilde{d_{i}^{U}}\right), H_{1}\left(\tilde{d_{i}^{U}}\right)\right),\left(d_{i 1}^{L}, d_{i 2}^{L}, d_{i 3}^{L}, d_{i 4}^{L} ; H_{1}\left(\tilde{d_{i}^{L}}\right), H_{1}\left(\tilde{d_{i}^{L}}\right)\right)\right) \\
& =R_{\text {rate }} x\left(\left(c_{i 1}^{U}, c_{i 2}^{U}, c_{i 3}^{U}, c_{i 4}^{U} ; H_{1}\left(\tilde{c_{i}^{U}}\right), H_{1}\left(\tilde{c_{i}^{U}}\right)\right),\left(c_{i 1}^{L}, c_{i 2}^{L}, c_{i 3}^{L}, c_{i 4}^{L} ; H_{1}\left(\tilde{c_{i}^{L}}\right), H_{1}\left(\tilde{c_{i}^{L}}\right)\right)\right)
\end{aligned}
$$

$\dot{C}_{i}, \dot{P}_{i}$ and $\dot{S}_{i}$ are defined for trapezoidal IT2FSs as follows in Eq. (45-47):

$$
\tilde{\tilde{C}}_{i}=\left(\left(c_{i 1}^{U}, c_{i 2}^{U}, c_{i 3}^{U}, c_{i 4}^{U} ; H_{1}\left(\tilde{c_{i}^{U}}\right), H_{1}\left(\tilde{c_{i}^{U}}\right)\right),\left(c_{i 1}^{L}, c_{i 2}^{L}, c_{i 3}^{L}, c_{i 4}^{L} ; H_{1}\left(\tilde{c_{i}^{L}}\right), H_{1}\left(\tilde{c_{i}^{L}}\right)\right)\right)
$$




$$
\begin{aligned}
\tilde{\tilde{P}}_{i}= & \left(\left(p_{i 1}^{U}, p_{i 2}^{U}, p_{i 3}^{U}, p_{i 4}^{U} ; H_{1}\left(\tilde{p_{i}^{U}}\right), H_{1}\left(\tilde{p_{i}^{U}}\right)\right),\left(p_{i 1}^{L}, p_{i 2}^{L}, p_{i 3}^{L}, p_{i 4}^{L} ; H_{1}\left(\tilde{p_{i}^{L}}\right), H_{1}\left(\tilde{p_{i}^{L}}\right)\right)\right) \\
\tilde{S}_{i}= & \left(\left(s_{i 1}^{U}, s_{i 2}^{U}, s_{i 3}^{U}, s_{i 4}^{U} ; H_{1}\left(\tilde{s_{i}^{U}}\right), H_{1}\left(\tilde{s_{i}^{U}}\right)\right),\left(s_{i 1}^{L}, s_{i 2}^{L}, s_{i 3}^{L}, s_{i 4}^{L} ; H_{1}\left(\tilde{s_{i}^{L}}\right), H_{1}\left(\tilde{s_{i}^{L}}\right)\right)\right) \\
= & L D T_{\text {displacement }}\left(\left(p_{i 1}^{U}, p_{i 2}^{U}, p_{i 3}^{U}, p_{i 4}^{U} ; H_{1}\left(\tilde{p_{i}^{U}}\right), H_{1}\left(\tilde{p_{i}^{U}}\right)\right),\right. \\
& \left.\left(\left(p_{i 1}^{L}, p_{i 2}^{L}, p_{i 3}^{L}, p_{i 4}^{L} ; H_{1}\left(\tilde{p_{i}^{L}}\right), H_{1}\left(\tilde{p_{i}^{L}}\right)\right)\right)\right)
\end{aligned}
$$

\section{APPLICATION}

The application of straight-line deprecation and double-declining balance depreciation methods are related to a ship in maritime. In application, classic, trapezoidal and triangular type-2 fuzzy straight-line deprecation and double-declining balance depreciation methods are calculated separately as below. The ship's characteristics are detailed in Table 1.

\subsection{Classic Straight-Line Depreciation Method}

The classic straight-line method is calculated from Eq. (22) as below follows:

$$
\begin{aligned}
D_{\text {deprecation }} & =\frac{1}{L_{\text {life }}}\left(C_{\text {cost }}-L D T_{\text {displacement }} P_{\text {price }}\right) \\
& =\frac{1}{25} x(23.000 .000-9.360 \times 350) \\
& =788.960
\end{aligned}
$$

In Table 1 and Table 2, the classic straight-line method of depreciation is calculated by breaking down the useful life of the ship.

\subsubsection{Triangular Interval Type-2 Fuzzy Straight-Line Depreciation Method}

Triangular Interval Type-2 Fuzzy depreciation under straight-line method of depreciation is calculated from $E q$. (26) as below follows:

$$
\begin{aligned}
& \dot{\dot{D}}_{\text {deprecation }}=\frac{1}{L_{\text {life }}}\left(\dot{;}_{\text {cos } t}-L D T_{\text {displacement }} \stackrel{\dot{P}}{\text { price }}\right) \\
& =\frac{1}{15} x(((22.000 .000,23.000 .000,24.000 .000 ; 1) \text {, } \\
& (22.250 .000,23.000 .000,23.750 .000 ; 0.9))- \\
& 9.360 x((250,350,450 ; 1),(275,350,425 ; 0.9))) \\
& =((711.520,788.960,866.400 ; 1),(730.880,788.960,847.040 ; 0.9))
\end{aligned}
$$

Triangular interval type-2 fuzzy depreciation under straight-line method of depreciation is defuzzied by calculating from $E q .(21)$ as below follows:

$$
\begin{aligned}
D_{i}^{U} & =711.520+(((866.400-711.520)+(788.960-711.520)) / 3) \\
& =788.960
\end{aligned}
$$




$$
\begin{aligned}
D_{i}^{L} & =730.880+(((847.040-730.880)+(788.960-730.880)) / 3) \\
& =788.960
\end{aligned}
$$

Value of $D_{11}^{U}$ and value of $D_{11}^{L}$ are computed by the arithmetic mean and crisp value of $D$ is computed at the end.

$$
\begin{aligned}
\tilde{D}_{i} & =\left(\frac{\tilde{D_{i}^{U}}+\tilde{D_{i}^{L}}}{2}\right) \\
& =\left(\frac{788.960+788.960}{2}\right) \\
& =788.960
\end{aligned}
$$

\begin{tabular}{|c|c|c|c|}
\hline Year & Book Value at Beginning of Year & Depreciation Expense & Accumulated Depreciation \\
\hline 1 & $\begin{array}{l}((22.000 .000,23.000 .000,24.000 .000 ; 1) \\
(22.250 .000,23.000 .000,23.750 .000 ; 0.9))\end{array}$ & $\begin{array}{l}((711.520,788.960,866.400 ; 1) \\
(730.880,788.960,847.040 ; 0.9))\end{array}$ & $\begin{array}{l}((711.520,788.960,866.400 ; 1) \\
(730.880,788.960,847.040 ; 0.9))\end{array}$ \\
\hline 2 & $\begin{array}{l}((21.133 .600,22.211 .040,23.288 .480 ; 1) \\
(21.402 .960,22.211 .040,23.019 .120 ; 0.9))\end{array}$ & $\begin{array}{l}((711.520,788.960,866.400 ; 1) \\
(730.880,788.960,847.040 ; 0.9))\end{array}$ & $\begin{array}{l}((1.423 .040,1.577 .920,1.732 .800 ; 1) \\
(1.461 .760,1.577 .920,1.694 .080 ; 0.9))\end{array}$ \\
\hline 3 & $\begin{array}{l}((20.267 .200,21.422 .080,22.576 .960 ; 1) \\
(20.555 .920,21.422 .080,22.288 .240 ; 0.9))\end{array}$ & $\begin{array}{l}((711.520,788.960,866.400 ; 1) \\
(730.880,788.960,847.040 ; 0.9))\end{array}$ & $\begin{array}{l}((2.134 .560,2.366 .880,2.599 .200 ; 1) \\
(2.192 .640,2.366 .880,2.541 .120 ; 0.9))\end{array}$ \\
\hline 4 & $\begin{array}{l}((19.400 .800,20.633 .120,21.865 .440 ; 1) \\
(19.708 .880,20.633 .120,21.557 .360 ; 0.9))\end{array}$ & $\begin{array}{l}((711.520,788.960,866.400 ; 1) \\
(730.880,788.960,847.040 ; 0.9))\end{array}$ & $\begin{array}{l}((2.846 .080,3.155 .840,3.465 .600 ; 1), \\
(2.923 .520,3.155 .840,3.388 .160 ; 0.9))\end{array}$ \\
\hline 5 & $\begin{array}{l}((18.534 .400,19.844 .160,21.153 .920 ; 1) \\
(18.861 .840,19.844 .160,20.826 .480 ; 0.9))\end{array}$ & $\begin{array}{l}((711.520,788.960,866.400 ; 1) \\
(730.880,788.960,847.040 ; 0.9))\end{array}$ & $\begin{array}{l}((3.557 .600,3.944 .800,4.332 .000 ; 1), \\
(3.654 .400,3.944 .800,4.235 .200 ; 0.9))\end{array}$ \\
\hline 6 & $\begin{array}{l}((17.668 .000,19.055 .200,20.442 .400 ; 1) \\
(18.014 .800,19.055 .200,20.095 .600 ; 0.9))\end{array}$ & $\begin{array}{l}((711.520,788.960,866.400 ; 1) \\
(730.880,788.960,847.040 ; 0.9))\end{array}$ & $\begin{array}{l}((4.269 .120,4.733 .760,5.198 .400 ; 1) \\
(4.385 .280,4.733 .760,5.082 .240 ; 0.9))\end{array}$ \\
\hline 7 & $\begin{array}{l}((16.801 .600,18.266 .240,19.730 .880 ; 1) \\
(17.167 .760,18.266 .240,19.364 .720 ; 0.9))\end{array}$ & $\begin{array}{l}((711.520,788.960,866.400 ; 1) \\
(730.880,788.960,847.040 ; 0.9))\end{array}$ & $\begin{array}{l}((4.980 .640,5.522 .720,6.064 .800 ; 1) \\
(5.116 .160,5.522 .720,5.929 .280 ; 0.9))\end{array}$ \\
\hline 8 & $\begin{array}{l}((15.935 .200,17.477 .280,19.019 .360 ; 1) \\
(16.320 .720,17.477 .280,18.633 .840 ; 0.9))\end{array}$ & $\begin{array}{l}((711.520,788.960,866.400 ; 1) \\
(730.880,788.960,847.040 ; 0.9))\end{array}$ & $\begin{array}{l}((5.692 .160,6.311 .680,6.931 .200 ; 1) \\
(5.847 .040,6.311 .680,6.776 .320 ; 0.9))\end{array}$ \\
\hline 9 & $\begin{array}{l}((15.068 .800,16.688 .320,18.307 .840 ; 1) \\
(15.473 .680,16.688 .320,17.902 .960 ; 0.9))\end{array}$ & $\begin{array}{l}((711.520,788.960,866.400 ; 1) \\
(730.880,788.960,847.040 ; 0.9))\end{array}$ & $\begin{array}{l}((6.403 .680,7.100 .640,7.797 .600 ; 1), \\
(6.577 .920,7.100 .640,7.623 .360 ; 0.9))\end{array}$ \\
\hline 10 & $\begin{array}{l}((14.202 .400,15.899 .360,17.596 .320 ; 1) \\
(14.626 .640,15.899 .360,17.172 .080 ; 0.9))\end{array}$ & $\begin{array}{l}((711.520,788.960,866.400 ; 1) \\
(730.880,788.960,847.040 ; 0.9))\end{array}$ & $\begin{array}{l}((7.115 .200,7.889 .600,8.664 .000 ; 1) \\
(7.308 .800,7.889 .600,8.470 .400 ; 0.9))\end{array}$ \\
\hline 11 & $\begin{array}{l}((13.336 .000,15.110 .400,16.884 .800 ; 1) \\
(13.779 .600,15.110 .400,16.441 .200 ; 0.9))\end{array}$ & $\begin{array}{l}((711.520,788.960,866.400 ; 1) \\
(730.880,788.960,847.040 ; 0.9))\end{array}$ & $\begin{array}{l}((7.826 .720,8.678 .560,9.530 .400 ; 1) \\
(8.039 .680,8.678 .560,9.317 .440 ; 0.9))\end{array}$ \\
\hline 12 & $\begin{array}{l}((12.469 .600,14.321 .440,16.173 .280 ; 1) \\
(12.932 .560,14.321 .440,15.710 .320 ; 0.9))\end{array}$ & $\begin{array}{l}((711.520,788.960,866.400 ; 1) \\
(730.880,788.960,847.040 ; 0.9))\end{array}$ & $\begin{array}{l}((8.538 .240,9.467 .520,10.396 .800 ; 1) \\
(8.770 .560,9.467 .520,10.164 .480 ; 0.9))\end{array}$ \\
\hline 13 & $\begin{array}{l}((11.603 .200,13.532 .480,15.461 .760 ; 1) \\
(12.085 .520,13.532 .480,14.979 .440 ; 0.9))\end{array}$ & $\begin{array}{l}((711.520,788.960,866.400 ; 1) \\
(730.880,788.960,847.040 ; 0.9))\end{array}$ & $\begin{array}{l}((9.249 .760,10.256 .480,11.263 .200 ; 1) \\
(9.501 .440,10.256 .480,11.011 .520 ; 0.9))\end{array}$ \\
\hline 14 & $\begin{array}{l}((10.736 .800,12.743 .520,14.750 .240 ; 1) \\
(11.238 .480,12.743 .520,14.248 .560 ; 0.9))\end{array}$ & $\begin{array}{l}((711.520,788.960,866.400 ; 1) \\
(730.880,788.960,847.040 ; 0.9))\end{array}$ & $\begin{array}{l}((9.961 .280,11.045 .440,12.129 .600 ; 1) \\
(10.232 .320,11.045 .440,11.858 .560 ; 0.9))\end{array}$ \\
\hline 15 & $\begin{array}{l}((9.870 .400,11.954 .560,14.038 .720 ; 1) \\
(10.391 .440,11.954 .560,13.517 .680 ; 0.9))\end{array}$ & $\begin{array}{l}((711.520,788.960,866.400 ; 1) \\
(730.880,788.960,847.040 ; 0.9))\end{array}$ & $\begin{array}{l}((10.672 .800,11.834 .400,12.996 .000 ; 1) \\
(10.963 .200,11.834 .400,12.705 .600 ; 0.9))\end{array}$ \\
\hline 16 & $\begin{array}{l}((9.004 .000,11.165 .600,13.327 .200 ; 1) \\
(9.544 .400,11.165 .600,12.786 .800 ; 0.9))\end{array}$ & $\begin{array}{l}((711.520,788.960,866.400 ; 1) \\
(730.880,788.960,847.040 ; 0.9))\end{array}$ & $\begin{array}{l}((11.384 .320,12.623 .360,13.862 .400 ; 1), \\
(11.694 .080,12.623 .360,13.552 .640 ; 0.9))\end{array}$ \\
\hline 17 & $\begin{array}{l}((8.137 .600,10.376 .640,12.615 .680 ; 1), \\
(8.697 .360,10.376 .640,12.055 .920 ; 0.9))\end{array}$ & $\begin{array}{l}((711.520,788.960,866.400 ; 1) \\
(730.880,788.960,847.040 ; 0.9))\end{array}$ & $\begin{array}{l}((12.095 .840,13.412 .320,14.728 .800 ; 1), \\
(12.424 .960,13.412 .320,14.399 .680 ; 0.9))\end{array}$ \\
\hline 18 & $\begin{array}{l}((7.271 .200,9.587 .680,11.904 .160 ; 1) \\
(7.850 .320,9.587 .680,11.325 .040 ; 0.9))\end{array}$ & $\begin{array}{l}((711.520,788.960,866.400 ; 1) \\
(730.880,788.960,847.040 ; 0.9))\end{array}$ & $\begin{array}{l}((12.807 .360,14.201 .280,15.595 .200 ; 1) \\
(13.155 .840,14.201 .280,15.246 .720 ; 0.9))\end{array}$ \\
\hline 19 & $\begin{array}{l}((6.404 .800,8.798 .720,11.192 .640 ; 1) \\
(7.003 .280,8.798 .720,10.594 .160 ; 0.9))\end{array}$ & $\begin{array}{l}((711.520,788.960,866.400 ; 1) \\
(730.880,788.960,847.040 ; 0.9))\end{array}$ & $\begin{array}{l}((13.518 .880,14.990 .240,16.461 .600 ; 1), \\
(13.886 .720,14.990 .240,16.093 .760 ; 0.9))\end{array}$ \\
\hline 20 & $\begin{array}{l}((5.538 .400,8.009 .760,10.481 .120 ; 1) \\
(6.156 .240,8.009 .760,9.863 .280 ; 0.9))\end{array}$ & $\begin{array}{l}((711.520,788.960,866.400 ; 1) \\
(730.880,788.960,847.040 ; 0.9))\end{array}$ & $\begin{array}{l}((14.230 .400,15.779 .200,17.328 .000 ; 1) \\
(14.617 .600,15.779 .200,16.940 .800 ; 0.9))\end{array}$ \\
\hline 21 & $\begin{array}{l}((4.672 .000,7.220 .800,9.769 .600 ; 1) \\
(5.309 .200,7.220 .800,9.132 .400 ; 0.9))\end{array}$ & $\begin{array}{l}((711.520,788.960,866.400 ; 1) \\
(730.880,788.960,847.040 ; 0.9))\end{array}$ & $\begin{array}{l}((14.941 .920,16.568 .160,18.194 .400 ; 1), \\
(15.348 .480,16.568 .160,17.787 .840 ; 0.9))\end{array}$ \\
\hline 22 & $\begin{array}{l}((3.805 .600,6.431 .840,9.058 .080 ; 1) \\
(4.462 .160,6.431 .840,8.401 .520 ; 0.9))\end{array}$ & $\begin{array}{l}((711.520,788.960,866.400 ; 1) \\
(730.880,788.960,847.040 ; 0.9))\end{array}$ & $\begin{array}{l}((15.653 .440,17.357 .120,19.060 .800 ; 1), \\
(16.079 .360,17.357 .120,18.634 .880 ; 0.9))\end{array}$ \\
\hline 23 & $\begin{array}{l}((2.939 .200,5.642 .880,8.346 .560 ; 1) \\
(3.615 .120,5.642 .880,7.670 .640 ; 0.9))\end{array}$ & $\begin{array}{l}((711.520,788.960,866.400 ; 1) \\
(730.880,788.960,847.040 ; 0.9))\end{array}$ & $\begin{array}{l}((16.364 .960,18.146 .080,19.927 .200 ; 1) \\
(16.810 .240,18.146 .080,19.481 .920 ; 0.9))\end{array}$ \\
\hline 24 & $\begin{array}{l}((2.072 .800,4.853 .920,7.635 .040 ; 1) \\
(2.768 .080,4.853 .920,6.939 .760 ; 0.9))\end{array}$ & $\begin{array}{l}((711.520,788.960,866.400 ; 1) \\
(730.880,788.960,847.040 ; 0.9))\end{array}$ & $\begin{array}{l}((17.076 .480,18.935 .040,20.793 .600 ; 1) \\
(17.541 .120,18.935 .040,20.328 .960 ; 0.9))\end{array}$ \\
\hline 25 & $\begin{array}{l}((1.206 .400,4.064 .960,6.923 .520 ; 1) \\
(1.921 .040,4.064 .960,6.208 .880 ; 0.9))\end{array}$ & $\begin{array}{l}((711.520,788.960,866.400 ; 1) \\
(730.880,788.960,847.040 ; 0.9))\end{array}$ & $\begin{array}{l}((17.788 .000,19.724 .000,21.660 .000 ; 1) \\
(18.272 .000,19.724 .000,21.176 .000 ; 0.9))\end{array}$ \\
\hline
\end{tabular}

In Table 3: Details of breakdown for triangular interval type-2 fuzzy for Straight-Line depreciation method

Table 4, the classic Straight-Line Method of Depreciation is calculated by breaking down for useful life of the ship. 


\subsubsection{Trapezoidal Interval Type-2 Fuzzy Straight-Line Method Depreciation}

Trapezoidal Interval Type-2 Fuzzy depreciation under straight-line method of depreciation is calculated from $E q$. (27) as below follows:

$$
\begin{aligned}
\dot{D}_{\text {deprecation }}= & \frac{1}{L_{\text {life }}}\left(\dot{\dot{C}}_{\text {cost }}-L D T_{\text {displacement }} \stackrel{\dot{P}}{\text { price }}\right) \\
= & \frac{1}{25} x(((22.000 .000,22.500 .000,23.500 .000,24.000 .000 ; 1,1), \\
& (22.250 .000,22.750 .000,23.250 .000,23.750 .000 ; 0.9,0.9))- \\
& 9.360 x((250,300,400,450 ; 1,1),(275,325,375,425 ; 0.9,0.9))) \\
= & ((711.520,750.240,827.680,866.400 ; 1,1), \\
& (730.880,769.600,808.320,847.040 ; 0.9,0.9))
\end{aligned}
$$

Trapezoidal Interval Type-2 Fuzzy depreciation under straight-line method of depreciation is defuzzied by calculating from $E q$. (20) as below follows:

$$
\begin{aligned}
D_{i}^{U}= & ((827.680 \times 866.400-711.520 \times 750.240)+ \\
& \left.\left((866.400-827.680)^{2}-(750.240-711.520)^{2}\right) / 3\right) /(827.680+866.400 \\
& -711.520-750.240) \\
= & 788.960 \\
D_{i}^{L}= & ((808.320 \times 847.040-730.880 \times 769.600)+ \\
& \left.\left((847.040-808.320)^{2}-(769.600-730.880)^{2}\right) / 3\right) /(808.320+847.040 \\
& -730.880-769.600) \\
= & 788.960
\end{aligned}
$$

Value of $D_{i}^{U}$ and value of $D_{i}^{L}$ are computed by the arithmetic mean and crisp value of $D$ is computed at the end.

$$
\begin{aligned}
\tilde{D}_{i} & =\left(\frac{\tilde{D_{i}^{U}}+\tilde{D_{i}^{L}}}{2}\right) \\
& =\left(\frac{788.960+788.960}{2}\right) \\
& =788.960
\end{aligned}
$$

In Table 4, the classic Straight-Line Method of Depreciation is calculated by breaking down for useful life of the ship.

\subsection{Double Declining Balance Depreciation Method}

Classic double-declining balance depreciation method is calculated from Eq. (37) as below follows:

$$
\begin{aligned}
D_{\text {deprecation }} & =R_{\text {rate }} x C_{\text {cost }} \\
& =8.00 \% \times 23.000 .000 \\
& =1.840 .000
\end{aligned}
$$


The double-declining balance depreciation rate was calculated by Eq. (38).

$$
\begin{aligned}
R_{\text {rate }} & =\left(\frac{100 \%}{L_{\text {life }}}\right) \times 2 \\
& =\left(\frac{100 \%}{25}\right) \times 2 \\
& =8.00 \%
\end{aligned}
$$

Salvage was calculated by Eq. (39).

$$
\begin{aligned}
S_{\text {salvage }} & =L D T_{\text {displacement }} P_{\text {price }} \\
& =9.360 \times 350 \\
& =3.276 .000
\end{aligned}
$$

In Table 1 and Table 5, the classic double-declining balance depreciation method is calculated by breaking down the useful life of the ship.

\subsubsection{Triangular Interval Type-2 Fuzzy Double Declining Balance Depreciation Method}

Triangular Interval Type-2 Fuzzy depreciation under double-declining balance method of depreciation is calculated from $E q$. (26) as below follows:

$$
\begin{aligned}
\dot{\dot{D}}_{\text {deprecation }}= & R_{\text {rate }} x \dot{\dot{C}}_{\cos t} \\
= & 8.00 \% x((22.000 .000,23.000 .000,24.000 .000 ; 1), \\
& (22.250 .000,23.000 .000,23.750 .000 ; 0.9)) \\
= & ((1.760 .000,1.840 .000,1.920 .000 ; 1), \\
& (1.780 .000,1.840 .000,1.900 .000 ; 0.9))
\end{aligned}
$$

Triangular interval type-2 fuzzy depreciation under double-declining balance method of depreciation is defuzzied by calculating from $E q$. (21) as below follows:

$$
\begin{aligned}
D_{i}^{U} & =1.760 .000+(((1.920 .000-1.760 .000)+(1.840 .000-1.760 .000)) / 3) \\
& =1.840 .000 \\
D_{i}^{L} & =1.780 .000+(((1.900 .000-1.780 .000)+(1.840 .000-1.780 .000)) / 3) \\
& =1.840 .000
\end{aligned}
$$

Value of $D_{11}^{U}$ and value of $D_{11}^{L}$ are computed by the arithmetic mean and crisp value of $D$ is computed at the end.

$$
\begin{aligned}
\tilde{D}_{i} & =\left(\frac{\tilde{D_{i}^{U}}+\tilde{D_{i}^{L}}}{2}\right) \\
& =\left(\frac{1.840 .000+1.840 .000}{2}\right) \\
& =1.840 .000
\end{aligned}
$$


In Table 6, the classic double-declining balance depreciation method is calculated by breaking down the useful life of the ship.

Trapezoidal Interval Type-2 Fuzzy depreciation of salvage for the ship is calculated from Eq. (39) as follows. The salvage calculation for both methods is the same.

$$
\begin{aligned}
S_{\text {salvage }}= & L D T_{\text {displacement }} P_{\text {price }} \\
= & 9.360 x((250,350,450 ; 1),(275,350,425 ; 0.9)) \\
= & ((2.340 .000,2.808 .000,3.744 .000,4.212 .000 ; 1,1), \\
& ((2.340 .000,3.276 .000,4.212 .000 ; 1),(2.574 .000,3.276 .000,3.978 .000 ; 0.9)) \\
= & 3.276 .000
\end{aligned}
$$

\subsubsection{Trapezoidal Interval Type-2 Fuzzy Double Declining Balance Depreciation Method}

Trapezoidal Interval Type-2 Fuzzy depreciation under double-declining balance method is calculated from $E q$. (37) as below follows:

$$
\begin{aligned}
\dot{\dot{D}}_{\text {deprecation }}= & R_{\text {rate }} x \dot{\dot{C}}_{\text {cost }} \\
= & 8.00 \% x(((22.000 .000,22.500 .000,23.500 .000,24.000 .000 ; 1,1), \\
& (22.250 .000,22.750 .000,23.250 .000,23.750 .000 ; 0.9,0.9)) \\
= & ((1.760 .000,1.800 .000,1.880 .000,1.920 .000 ; 1,1), \\
& (1.780 .000,1.820 .000,1.860 .000,1.900 .000 ; 0.9,0.9))
\end{aligned}
$$

Trapezoidal Interval Type-2 Fuzzy depreciation under straight-line method of depreciation is defuzzied by calculating from $E q$. (20) as below follows:

$$
\begin{aligned}
D_{i}^{U}= & ((1.880 .000 \times 1.920 .000-1.760 .000 \times 1.800 .000)+ \\
& \left.\left((1.880 .000-1.920 .000)^{2}-(1.760 .000-1.800 .000)^{2}\right) / 3\right) /(1.880 .000+1.920 .000 \\
& -1.760 .000-1.800 .000) \\
= & 1.840 .000 \\
D_{i}^{L}= & ((1.860 .000 \times 1.900 .000-1.780 .000 \times 1.820 .000)+ \\
& \left.\left(1.860 .000-1.900 .000^{2}-(1.780 .000-1.820 .000)^{2}\right) / 3\right) / 1.860 .000+1.900 .000 \\
& -1.780 .000-1.820 .000) \\
= & 1.840 .000
\end{aligned}
$$

Value of $D_{i}^{U}$ and value of $D_{i}^{L}$ are computed by the arithmetic mean and crisp value of $D$ is computed at the end. 


$$
\begin{aligned}
\dot{D}_{i} & =\left(\frac{\vdots}{\vdots} \begin{array}{c}
\vdots \\
D_{i}^{U}+D_{i}^{L} \\
2
\end{array}\right) \\
& =\left(\frac{1.840 .000+1.840 .000}{2}\right) \\
& =1.840 .000
\end{aligned}
$$

In Table 7, the classic double-declining balance depreciation method is calculated by breaking down the useful life of the ship.

Trapezoidal Interval Type-2 Fuzzy depreciation of salvage for the ship is calculated from Eq. (39) as follows. The salvage calculation for both methods is the same.

$$
\begin{aligned}
S_{\text {salvage }}= & L D T_{\text {displacement }} P_{\text {price }} \\
= & 9.360 x((250,300,400,450 ; 1,1),(275,325,375,425 ; 0.9,0.9)) \\
= & ((2.340 .000,2.808 .000,3.744 .000,4.212 .000 ; 1,1), \\
& (2.574 .000,3.042 .000,3.510 .000,3.978 .000 ; 0.9,0.9)) \\
= & 3.276 .000
\end{aligned}
$$


Table 1: Details of deprecation for the ship

\begin{tabular}{|c|c|c|c|}
\hline $\begin{array}{l}\text { Items of } \\
\text { Deprecation }\end{array}$ & $\begin{array}{r}\text { Classic } \\
\text { Deprecation } \\
\end{array}$ & $\begin{array}{r}\text { Trapezoidal Interval Type-2 Fuzzy } \\
\text { Deprecation }\end{array}$ & $\begin{array}{r}\text { Triangular Interval Type-2 Fuzzy } \\
\text { Deprecation }\end{array}$ \\
\hline Price of Assets $(U S D)$ & 23.000 .000 & $\begin{array}{r}((22.000 .000,22.500 .000,23.500 .000,24.000 .000 ; 1,1) \\
(22.250 .000,22.750 .000,23.250 .000,23.750 .000 ; 0.9,0.9))\end{array}$ & $\begin{array}{r}((22.000 .000,23.000 .000,24.000 .000 ; 1), \\
(22.250 .000,23.000 .000,23.750 .000 ; 0.9))\end{array}$ \\
\hline Price of Scrap Metal $(U S D)$ & 350 & $\begin{array}{r}((250,300,400,450 ; 1,1) \\
(275,325,375,425 ; 0.9,0.9))\end{array}$ & $\begin{array}{r}((250,350,450 ; 1) \\
(275,350,425 ; 0.9))\end{array}$ \\
\hline Light Ship Displacement (LDT) & 9.360 & 9.360 & 9.360 \\
\hline Salvage Value $(U S D)$ & 3.276 .000 & $\begin{array}{r}((2.340 .000,2.808 .000,3.744 .000,4.212 .000 ; 1,1), \\
(2.574 .000,3.042 .000,3.510 .000,3.978 .000 ; 0.9,0.9))\end{array}$ & $\begin{array}{r}((2.340 .000,3.276 .000,4.212 .000 ; 1) \\
(2.574 .000,3.276 .000,3.978 .000 ; 0.9))\end{array}$ \\
\hline Utilization Life (Year) & 25 & 25 & 25 \\
\hline Useful Life (Year) & 25 & 25 & 25 \\
\hline Age of Asset (Year) & 0 & 0 & 0 \\
\hline Annual Depreciation & 788.960 & $\begin{array}{r}((711.520,750.240,827.680,866.400 ; 1,1) \\
(730.880,769.600,808.320,847.040 ; 0.9,0.9))\end{array}$ & $\begin{array}{r}((711.520,788.960,866.400 ; 1) \\
(730.880,788.960,847.040 ; 0.9))\end{array}$ \\
\hline Annual Deprecation Defuzzfy & 788.960 & 788.960 & 788.960 \\
\hline $\begin{array}{l}\text { Double Declining Balance } \\
\text { Depreciation Rate }(\%)\end{array}$ & $8.00 \%$ & $8.00 \%$ & $8.00 \%$ \\
\hline
\end{tabular}


Table 2: Details of breakdown for classic Straight-Line depreciation method.

\begin{tabular}{|c|c|c|c|c|}
\hline Year & Book Value at Beginning of Year & Depreciation Expense & Accumulated Depreciation & Book Value at End of Year \\
\hline 1 & 23.000 .000 & 788.960 & 788.960 & 22.211 .040 \\
\hline 2 & 22.211 .040 & 788.960 & 1.577 .920 & 21.422 .080 \\
\hline 3 & 21.422 .080 & 788.960 & 2.366 .880 & 20.633 .120 \\
\hline 4 & 20.633 .120 & 788.960 & 3.155 .840 & 19.844 .160 \\
\hline 5 & 19.844 .160 & 788.960 & 3.944 .800 & 19.055 .200 \\
\hline 6 & 19.055 .200 & 788.960 & 4.733 .760 & 18.266 .240 \\
\hline 7 & 18.266 .240 & 788.960 & 5.522 .720 & 17.477 .280 \\
\hline 8 & 17.477 .280 & 788.960 & 6.311 .680 & 16.688 .320 \\
\hline 9 & 16.688 .320 & 788.960 & 7.100 .640 & 15.899 .360 \\
\hline 10 & 15.899 .360 & 788.960 & 7.889 .600 & 15.110 .400 \\
\hline 11 & 15.110 .400 & 788.960 & 8.678 .560 & 14.321 .440 \\
\hline 12 & 14.321 .440 & 788.960 & 9.467 .520 & 13.532 .480 \\
\hline 13 & 13.532 .480 & 788.960 & 10.256 .480 & 12.743 .520 \\
\hline 14 & 12.743 .520 & 788.960 & 11.045 .440 & 11.954 .560 \\
\hline 15 & 11.954 .560 & 788.960 & 11.834 .400 & 11.165 .600 \\
\hline 16 & 11.165 .600 & 788.960 & 12.623 .360 & 10.376 .640 \\
\hline 17 & 10.376 .640 & 788.960 & 13.412 .320 & 9.587 .680 \\
\hline 18 & 9.587 .680 & 788.960 & 14.201 .280 & 8.798 .720 \\
\hline 19 & 8.798 .720 & 788.960 & 14.990 .240 & 8.009 .760 \\
\hline 20 & 8.009 .760 & 788.960 & 15.779 .200 & 7.220 .800 \\
\hline 21 & 7.220 .800 & 788.960 & 16.568 .160 & 6.431 .840 \\
\hline 22 & 6.431 .840 & 788.960 & 17.357 .120 & 5.642 .880 \\
\hline 23 & 5.642 .880 & 788.960 & 18.146 .080 & 4.853 .920 \\
\hline 24 & 4.853 .920 & 788.960 & 18.935 .040 & 4.064 .960 \\
\hline 25 & 4.064 .960 & 788.960 & 19.724 .000 & 3.276 .000 \\
\hline
\end{tabular}


Table 3: Details of breakdown for triangular interval type-2 fuzzy for Straight-Line depreciation method

\begin{tabular}{|c|c|c|c|c|}
\hline Year & Book Value at Beginning of Year & Depreciation Expense & Accumulated Depreciation & Book Value at End of Year \\
\hline 1 & $\begin{array}{l}((22.000 .000,23.000 .000,24.000 .000 ; 1) \\
(22.250 .000,23.000 .000,23.750 .000 ; 0.9))\end{array}$ & $\begin{array}{l}((711.520,788.960,866.400 ; 1), \\
(730.880,788.960,847.040 ; 0.9))\end{array}$ & $\begin{array}{l}((711.520,788.960,866.400 ; 1), \\
(730.880,788.960,847.040 ; 0.9))\end{array}$ & $\begin{array}{l}((21.133 .600,22.211 .040,23.288 .480 ; 1), \\
(21.402 .960,22.211 .040,23.019 .120 ; 0.9))\end{array}$ \\
\hline 2 & $\begin{array}{l}((21.133 .600,22.211 .040,23.288 .480 ; 1) \\
(21.402 .960,22.211 .040,23.019 .120 ; 0.9))\end{array}$ & $\begin{array}{l}((711.520,788.960,866.400 ; 1) \\
(730.880,788.960,847.040 ; 0.9))\end{array}$ & $\begin{array}{l}((1.423 .040,1.577 .920,1.732 .800 ; 1) \\
(1.461 .760,1.577 .920,1.694 .080 ; 0.9))\end{array}$ & $\begin{array}{l}((20.267 .200,21.422 .080,22.576 .960 ; 1) \\
(20.555 .920,21.422 .080,22.288 .240 ; 0.9))\end{array}$ \\
\hline 3 & $\begin{array}{l}((20.267 .200,21.422 .080,22.576 .960 ; 1) \\
(20.555 .920,21.422 .080,22.288 .240 ; 0.9))\end{array}$ & $\begin{array}{l}((711.520,788.960,866.400 ; 1), \\
(730.880,788.960,847.040 ; 0.9))\end{array}$ & $\begin{array}{l}((2.134 .560,2.366 .880,2.599 .200 ; 1) \\
(2.192 .640,2.366 .880,2.541 .120 ; 0.9))\end{array}$ & $\begin{array}{l}((19.400 .800,20.633 .120,21.865 .440 ; 1), \\
(19.708 .880,20.633 .120,21.557 .360 ; 0.9))\end{array}$ \\
\hline 4 & $\begin{array}{l}((19.400 .800,20.633 .120,21.865 .440 ; 1) \\
(19.708 .880,20.633 .120,21.557 .360 ; 0.9))\end{array}$ & $\begin{array}{l}((711.520,788.960,866.400 ; 1), \\
(730.880,788.960,847.040 ; 0.9))\end{array}$ & $\begin{array}{l}((2.846 .080,3.155 .840,3.465 .600 ; 1), \\
(2.923 .520,3.155 .840,3.388 .160 ; 0.9))\end{array}$ & $\begin{array}{l}((18.534 .400,19.844 .160,21.153 .920 ; 1), \\
(18.861 .840,19.844 .160,20.826 .480 ; 0.9))\end{array}$ \\
\hline 5 & $\begin{array}{l}((18.534 .400,19.844 .160,21.153 .920 ; 1) \\
(18.861 .840,19.844 .160,20.826 .480 ; 0.9))\end{array}$ & $\begin{array}{l}((711.520,788.960,866.400 ; 1), \\
(730.880,788.960,847.040 ; 0.9))\end{array}$ & $\begin{array}{l}((3.557 .600,3.944 .800,4.332 .000 ; 1), \\
(3.654 .400,3.944 .800,4.235 .200 ; 0.9))\end{array}$ & $\begin{array}{l}((17.668 .000,19.055 .200,20.442 .400 ; 1), \\
(18.014 .800,19.055 .200,20.095 .600 ; 0.9))\end{array}$ \\
\hline 6 & $\begin{array}{l}((17.668 .000,19.055 .200,20.442 .400 ; 1) \\
(18.014 .800,19.055 .200,20.095 .600 ; 0.9))\end{array}$ & $\begin{array}{l}((711.520,788.960,866.400 ; 1) \\
(730.880,788.960,847.040 ; 0.9))\end{array}$ & $\begin{array}{l}((4.269 .120,4.733 .760,5.198 .400 ; 1) \\
(4.385 .280,4.733 .760,5.082 .240 ; 0.9))\end{array}$ & $\begin{array}{l}((16.801 .600,18.266 .240,19.730 .880 ; 1), \\
(17.167 .760,18.266 .240,19.364 .720 ; 0.9))\end{array}$ \\
\hline 7 & $\begin{array}{l}((16.801 .600,18.266 .240,19.730 .880 ; 1), \\
(17.167 .760,18.266 .240,19.364 .720 ; 0.9))\end{array}$ & $\begin{array}{l}((711.520,788.960,866.400 ; 1), \\
(730.880,788.960,847.040 ; 0.9))\end{array}$ & $\begin{array}{l}((4.980 .640,5.522 .720,6.064 .800 ; 1), \\
(5.116 .160,5.522 .720,5.929 .280 ; 0.9))\end{array}$ & $\begin{array}{l}((15.935 .200,17.477 .280,19.019 .360 ; 1), \\
(16.320 .720,17.477 .280,18.633 .840 ; 0.9))\end{array}$ \\
\hline 8 & $\begin{array}{l}((15.935 .200,17.477 .280,19.019 .360 ; 1) \\
(16.320 .720,17.477 .280,18.633 .840 ; 0.9))\end{array}$ & $\begin{array}{l}((711.520,788.960,866.400 ; 1), \\
(730.880,788.960,847.040 ; 0.9))\end{array}$ & $\begin{array}{l}((5.692 .160,6.311 .680,6.931 .200 ; 1), \\
(5.847 .040,6.311 .680,6.776 .320 ; 0.9))\end{array}$ & $\begin{array}{l}((15.068 .800,16.688 .320,18.307 .840 ; 1), \\
(15.473 .680,16.688 .320,17.902 .960 ; 0.9))\end{array}$ \\
\hline 9 & $\begin{array}{l}((15.068 .800,16.688 .320,18.307 .840 ; 1) \\
(15.473 .680,16.688 .320,17.902 .960 ; 0.9))\end{array}$ & $\begin{array}{l}((711.520,788.960,866.400 ; 1), \\
(730.880,788.960,847.040 ; 0.9))\end{array}$ & $\begin{array}{l}((6.403 .680,7.100 .640,7.797 .600 ; 1), \\
(6.577 .920,7.100 .640,7.623 .360 ; 0.9))\end{array}$ & $\begin{array}{l}((14.202 .400,15.899 .360,17.596 .320 ; 1), \\
(14.626 .640,15.899 .360,17.172 .080 ; 0.9))\end{array}$ \\
\hline 10 & $\begin{array}{l}((14.202 .400,15.899 .360,17.596 .320 ; 1) \\
(14.626 .640,15.899 .360,17.172 .080 ; 0.9))\end{array}$ & $\begin{array}{l}((711.520,788.960,866.400 ; 1), \\
(730.880,788.960,847.040 ; 0.9))\end{array}$ & $\begin{array}{l}((7.115 .200,7.889 .600,8.664 .000 ; 1), \\
(7.308 .800,7.889 .600,8.470 .400 ; 0.9))\end{array}$ & $\begin{array}{l}((13.336 .000,15.110 .400,16.884 .800 ; 1), \\
(13.779 .600,15.110 .400,16.441 .200 ; 0.9))\end{array}$ \\
\hline 11 & $\begin{array}{l}((13.336 .000,15.110 .400,16.884 .800 ; 1), \\
(13.779 .600,15.110 .400,16.441 .200 ; 0.9))\end{array}$ & $\begin{array}{l}((711.520,788.960,866.400 ; 1), \\
(730.880,788.960,847.040 ; 0.9))\end{array}$ & $\begin{array}{l}((7.826 .720,8.678 .560,9.530 .400 ; 1), \\
(8.039 .680,8.678 .560,9.317 .440 ; 0.9))\end{array}$ & $\begin{array}{l}((12.469 .600,14.321 .440,16.173 .280 ; 1), \\
(12.932 .560,14.321 .440,15.710 .320 ; 0.9))\end{array}$ \\
\hline 12 & $\begin{array}{l}((12.469 .600,14.321 .440,16.173 .280 ; 1) \\
(12.932 .560,14.321 .440,15.710 .320 ; 0.9))\end{array}$ & $\begin{array}{l}((711.520,788.960,866.400 ; 1), \\
(730.880,788.960,847.040 ; 0.9))\end{array}$ & $\begin{array}{l}((8.538 .240,9.467 .520,10.396 .800 ; 1), \\
(8.770 .560,9.467 .520,10.164 .480 ; 0.9))\end{array}$ & $\begin{array}{l}((11.603 .200,13.532 .480,15.461 .760 ; 1), \\
(12.085 .520,13.532 .480,14.979 .440 ; 0.9))\end{array}$ \\
\hline 13 & $\begin{array}{l}((11.603 .200,13.532 .480,15.461 .760 ; 1) \\
(12.085 .520,13.532 .480,14.979 .440 ; 0.9))\end{array}$ & $\begin{array}{l}((711.520,788.960,866.400 ; 1), \\
(730.880,788.960,847.040 ; 0.9))\end{array}$ & $\begin{array}{l}((9.249 .760,10.256 .480,11.263 .200 ; 1) \\
(9.501 .440,10.256 .480,11.011 .520 ; 0.9))\end{array}$ & $\begin{array}{l}((10.736 .800,12.743 .520,14.750 .240 ; 1), \\
(11.238 .480,12.743 .520,14.248 .560 ; 0.9))\end{array}$ \\
\hline 14 & $\begin{array}{l}((10.736 .800,12.743 .520,14.750 .240 ; 1) \\
(11.238 .480,12.743 .520,14.248 .560 ; 0.9))\end{array}$ & $\begin{array}{l}((711.520,788.960,866.400 ; 1), \\
(730.880,788.960,847.040 ; 0.9))\end{array}$ & $\begin{array}{l}((9.961 .280,11.045 .440,12.129 .600 ; 1), \\
(10.232 .320,11.045 .440,11.858 .560 ; 0.9))\end{array}$ & $\begin{array}{l}((9.870 .400,11.954 .560,14.038 .720 ; 1), \\
(10.391 .440,11.954 .560,13.517 .680 ; 0.9))\end{array}$ \\
\hline 15 & $\begin{array}{l}((9.870 .400,11.954 .560,14.038 .720 ; 1), \\
(10.391 .440,11.954 .560,13.517 .680 ; 0.9))\end{array}$ & $\begin{array}{l}((711.520,788.960,866.400 ; 1), \\
(730.880,788.960,847.040 ; 0.9))\end{array}$ & $\begin{array}{l}((10.672 .800,11.834 .400,12.996 .000 ; 1), \\
(10.963 .200,11.834 .400,12.705 .600 ; 0.9))\end{array}$ & $\begin{array}{l}((9.004 .000,11.165 .600,13.327 .200 ; 1), \\
(9.544 .400,11.165 .600,12.786 .800 ; 0.9))\end{array}$ \\
\hline 16 & $\begin{array}{l}((9.004 .000,11.165 .600,13.327 .200 ; 1) \\
(9.544 .400,11.165 .600,12.786 .800 ; 0.9))\end{array}$ & $\begin{array}{l}((711.520,788.960,866.400 ; 1) \\
(730.880,788.960,847.040 ; 0.9))\end{array}$ & $\begin{array}{l}((11.384 .320,12.623 .360,13.862 .400 ; 1), \\
(11.694 .080,12.623 .360,13.552 .640 ; 0.9))\end{array}$ & $\begin{array}{l}((8.137 .600,10.376 .640,12.615 .680 ; 1), \\
(8.697 .360,10.376 .640,12.055 .920 ; 0.9))\end{array}$ \\
\hline 17 & $\begin{array}{l}((8.137 .600,10.376 .640,12.615 .680 ; 1), \\
(8.697 .360,10.376 .640,12.055 .920 ; 0.9))\end{array}$ & $\begin{array}{l}((711.520,788.960,866.400 ; 1), \\
(730.880,788.960,847.040 ; 0.9))\end{array}$ & $\begin{array}{l}((12.095 .840,13.412 .320,14.728 .800 ; 1), \\
(12.424 .960,13.412 .320,14.399 .680 ; 0.9))\end{array}$ & $\begin{array}{l}((7.271 .200,9.587 .680,11.904 .160 ; 1) \\
(7.850 .320,9.587 .680,11.325 .040 ; 0.9))\end{array}$ \\
\hline 18 & $\begin{array}{l}((7.271 .200,9.587 .680,11.904 .160 ; 1), \\
(7.850 .320,9.587 .680,11.325 .040 ; 0.9))\end{array}$ & $\begin{array}{l}((711.520,788.960,866.400 ; 1), \\
(730.880,788.960,847.040 ; 0.9))\end{array}$ & $\begin{array}{l}((12.807 .360,14.201 .280,15.595 .200 ; 1), \\
(13.155 .840,14.201 .280,15.246 .720 ; 0.9))\end{array}$ & $\begin{array}{l}((6.404 .800,8.798 .720,11.192 .640 ; 1), \\
(7.003 .280,8.798 .720,10.594 .160 ; 0.9))\end{array}$ \\
\hline 19 & $\begin{array}{l}((6.404 .800,8.798 .720,11.192 .640 ; 1), \\
(7.003 .280,8.798 .720,10.594 .160 ; 0.9))\end{array}$ & $\begin{array}{l}((711.520,788.960,866.400 ; 1), \\
(730.880,788.960,847.040 ; 0.9))\end{array}$ & $\begin{array}{l}((13.518 .880,14.990 .240,16.461 .600 ; 1), \\
(13.886 .720,14.990 .240,16.093 .760 ; 0.9))\end{array}$ & $\begin{array}{l}((5.538 .400,8.009 .760,10.481 .120 ; 1), \\
(6.156 .240,8.009 .760,9.863 .280 ; 0.9))\end{array}$ \\
\hline 20 & $\begin{array}{l}((5.538 .400,8.009 .760,10.481 .120 ; 1), \\
(6.156 .240,8.009 .760,9.863 .280 ; 0.9))\end{array}$ & $\begin{array}{l}((711.520,788.960,866.400 ; 1), \\
(730.880,788.960,847.040 ; 0.9))\end{array}$ & $\begin{array}{l}((14.230 .400,15.779 .200,17.328 .000 ; 1), \\
(14.617 .600,15.779 .200,16.940 .800 ; 0.9))\end{array}$ & $\begin{array}{l}((4.672 .000,7.220 .800,9.769 .600 ; 1) \\
(5.309 .200,7.220 .800,9.132 .400 ; 0.9))\end{array}$ \\
\hline 21 & $\begin{array}{l}((4.672 .000,7.220 .800,9.769 .600 ; 1) \\
(5.309 .200,7.220 .800,9.132 .400 ; 0.9))\end{array}$ & $\begin{array}{l}((711.520,788.960,866.400 ; 1), \\
(730.880,788.960,847.040 ; 0.9))\end{array}$ & $\begin{array}{l}((14.941 .920,16.568 .160,18.194 .400 ; 1), \\
(15.348 .480,16.568 .160,17.787 .840 ; 0.9))\end{array}$ & $\begin{array}{l}((3.805 .600,6.431 .840,9.058 .080 ; 1) \\
(4.462 .160,6.431 .840,8.401 .520 ; 0.9))\end{array}$ \\
\hline 22 & $\begin{array}{l}((3.805 .600,6.431 .840,9.058 .080 ; 1) \\
(4.462 .160,6.431 .840,8.401 .520 ; 0.9))\end{array}$ & $\begin{array}{l}((711.520,788.960,866.400 ; 1), \\
(730.880,788.960,847.040 ; 0.9))\end{array}$ & $\begin{array}{l}((15.653 .440,17.357 .120,19.060 .800 ; 1), \\
(16.079 .360,17.357 .120,18.634 .880 ; 0.9))\end{array}$ & $\begin{array}{l}((2.939 .200,5.642 .880,8.346 .560 ; 1) \\
(3.615 .120,5.642 .880,7.670 .640 ; 0.9))\end{array}$ \\
\hline 23 & $\begin{array}{l}((2.939 .200,5.642 .880,8.346 .560 ; 1) \\
(3.615 .120,5.642 .880,7.670 .640 ; 0.9))\end{array}$ & $\begin{array}{l}((711.520,788.960,866.400 ; 1), \\
(730.880,788.960,847.040 ; 0.9))\end{array}$ & $\begin{array}{l}((16.364 .960,18.146 .080,19.927 .200 ; 1), \\
(16.810 .240,18.146 .080,19.481 .920 ; 0.9))\end{array}$ & $\begin{array}{l}((2.072 .800,4.853 .920,7.635 .040 ; 1) \\
(2.768 .080,4.853 .920,6.939 .760 ; 0.9))\end{array}$ \\
\hline 24 & $\begin{array}{l}((2.072 .800,4.853 .920,7.635 .040 ; 1), \\
(2.768 .080,4.853 .920,6.939 .760 ; 0.9))\end{array}$ & $\begin{array}{l}((711.520,788.960,866.400 ; 1), \\
(730.880,788.960,847.040 ; 0.9))\end{array}$ & $\begin{array}{l}((17.076 .480,18.935 .040,20.793 .600 ; 1), \\
(17.541 .120,18.935 .040,20.328 .960 ; 0.9))\end{array}$ & $\begin{array}{l}((1.206 .400,4.064 .960,6.923 .520 ; 1), \\
(1.921 .040,4.064 .960,6.208 .880 ; 0.9))\end{array}$ \\
\hline 25 & $\begin{array}{l}((1.206 .400,4.064 .960,6.923 .520 ; 1) \\
(1.921 .040,4.064 .960,6.208 .880 ; 0.9))\end{array}$ & $\begin{array}{l}((711.520,788.960,866.400 ; 1), \\
(730.880,788.960,847.040 ; 0.9))\end{array}$ & $\begin{array}{l}((17.788 .000,19.724 .000,21.660 .000 ; 1), \\
(18.272 .000,19.724 .000,21.176 .000 ; 0.9))\end{array}$ & $\begin{array}{l}((340.000,3.276 .000,6.212 .000 ; 1), \\
(1.074 .000,3.276 .000,5.478 .000 ; 0.9))\end{array}$ \\
\hline
\end{tabular}


Table 4: Details of breakdown for trapezoidal interval type-2 fuzzy for Straight-Line depreciation method

Year Book Value at Beginning of Year

$((22.000 .000,22.500 .000,23.500 .000,24.000 .000 ; 1,1)$

(21.25.00, 22.750.000, 23.250.000, 23.750.000,0.9,

$2 \quad((21.133 .600,21.672 .320,22.749 .760,23.288 .480 ; 1,1)$, (20.267.200, 20.844.640,21.999.520, 22.576.960:1,1), (20.267.200, 20.844.640, 21.999.520, 22.576.960;1, 1) (19.400.800, 20.016.960, 21.249.280, 21.865.440:1, 1), (19.708.880,20.325.940, 20.241.200, 21.865.440,1, 1)

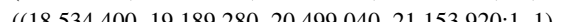
(18.861.840, 19.516.280, 20.171.600, 20.826.480;0.9, 0.9$)$ (17.668.000, $18.361 .600,19.748 .800,20.442 .400 ; 1,1)$ (18.014.800, 18.708.400, 19.402.800, 20.095.400:0.9, $((16.801 .600,17.533 .920,18.998 .560,19.730 .880: 1,1)$ $(17.167 .760,17.500 .080,18.632 .400,19.364 .720 ; 0.9,0.9)$ $((15.935 .200,16.706 .240,18.248 .320,19.019 .360 ; 1,1)$ $(16.320 .720,17.091 .760,17.862800,18.633 .840 \cdot 0.9,0.9))$ $((15.068 .800,15.878 .560,17.498080,18.307 .840: 1,1)$ $(15.473 .680,16283.440,17.093200,17.902 .960 \cdot 0.9,0.9)$ (14.202.400, 15.050.880.16.747.840,17.596.320:1,1)

$10 \quad(14.626 .640,15.475 .120,16.323 .600,17.172 .080 ; 0.9,0.9)$ $((13.336 .000,14.223 .200,15.997 .600,16.884 .800: 1,1)$ (13.779.600, 14.666.800, 15.554.000, 16.441.200.0.9, 0.9) ((12.469.600, 13.395.520, 15.247.360, 16.173.280:1, 1) (12.932.560, 13.858.480, 14.784.400, 15.710.320:0.9, 0.9) $((11.603 .200,12.567 .840,14.497 .120,15.461 .760 ; 1,1)$ $(12.085 .520,13.050 .160,14.014 .800,14.979 .440 \cdot 0.9,0.9)$ ((10.736.800, 11.740.160, 13.746.880, 14.750.240;1, 1) $(11.238 .480,12.241 .840,13.245 .200,14.248 .560 \cdot 0.9,0.9)$ $((9.870 .400,10.912 .480,12.996 .640,14.038 .720 ; 1,1)$, $(10.391 .440,11.433 .520,12.475 .600,13.517 .680 ; 0.9,0.9)$ $6 \quad((9.004 .000,10.084 .800,12.246 .400,13.327 .200 ; 1,1)$, ((8.137.600, $9.257 .120,11.496 .160,12.615 .680 \cdot 1,1)$. $(8.697 .360,9.816 .880,10.936 .400,12.055 .920 ; 0.9,0.9)$ ((7.271.200, 8.429.440, $10.745 .920,11.904 .160 ; 1,1)$ $(7.850 .320,9.008 .560,10.166 .800,11.325 .040 ; 0.9,0.9))$ ((6.404.800, 7.601.760, 9.995.680, 11.192.640:1, 1), $(7.003 .280,8.200 .240,9.397 .200,10.594 .160 ; 0.9,0.9))$ $((5.538 .400,6.774 .080,9.245 .440,10.481 .120 ; 1,1)$
$(6.156 .240,7.391 .920,8.627 .600,9.863 .280 ; 0.9,0.9))$ ((4.672.000, 5.946.400, 8.495.200, 9.769.600:1, 1), (5.309.200, 6.583.600, 7.858.000, 9.132.400;0.9, 0.9)) ((3.805.600, 5.118.720, 7.744.960, 9.058.080;1, 1) $(4.462 .160,5.775 .280,7.088 .400,8.401 .520 ; 0.9,0.9)$ $((2.939 .200,4.291 .040,6.994 .720,8.346 .560 ; 1,1)$ $(3.615 .120,4.966 .960,6.318 .800,7.670 .640 ; 0.9,0.9))$ $((2.072 .800,3.463 .360,6.244 .480,7.635 .040 ; 1,0)$ (2.768.080, 4.158.640, 5.549.200, 6.939.760;0.9, 0.9) ((1.206.400, 2.635.680, 5.494.240, 6.923.520;1,

\section{Depreciation Expense}

((711.520, 750.240, 827.680, 866.400;1, 1),
(711.520, 750.240, $827.680,866.400,1,1)$, (711.520, 750.240, 827.680,866.400:1, 1), (7) $(730.880,769.600,808.320,847.040,1,9)$, (711.520, 750.240, 827.680,866.400:1, 1), (730.880, 769.600,808.320,847.040,1,

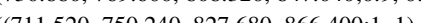
(730.880, 769.600, 808.320,847.040,0.9,0.9, (7) (711.520, 750.6240,827.680, $866.400: 1,1)$ (730.880, 769.600,808.320,847.040,1, (7) (711.520, $750.240,827.680,866.400 .1,0)$ $(730.880,769.600,808.320,847.040,0,9,0.9)$

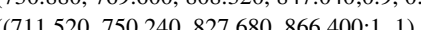
(730.880, 769.600, 808.320,847.040,1, $((711.520,750.240,827.680,866.400: 1,1)$ $(730.880,769.600,808.320,847.040 ; 0,9,0,9)$ $((711.520,750.240 .827 .680 .866 .400: 1,1)$ $(730.880,769.600,808.320,847.040 \cdot 0.9,0.9))$ $((711.520,750.240,827.680,866.400: 1,1)$ $(730.880,769.600,808.320,847.040 \cdot 0.9,0.9))$ $((711.520,750.240,827.680,866.400 ; 1,1)$ (730.880, 769.600, 808.320,847.040:0.9, 0.9 ) $((711.520,750.240,827.680,866.400: 1,1)$ $(730.880,769.600,808.320 .847 .040 \cdot 0.9,0.9))$ ((711.520, 750.240, 827.680, 866.400;1, 1), $(730.880,769.600,808.320,847.040 ; 0.9,0.9)$ $((711.520,750.240,827.680,866.400 ; 1,1)$, $(730.880,769.600,808.320,847.040 \cdot 0.9,0.9))$ ((711.520, 750.240, 827.680, 866.400;1. 1), $(730.880,769.600,808.320,847.040 ; 0.9,0.9))$ ((711.520, 750.240, 827.680, 866.400;1, 1) $(730.880,769.600,808.320,847.040 ; 0.9,0.9)$ ((711.520, 750.240, 827.680, 866.400;1, 1), $(730.880,769.600,808.320,847.040 ; 0.9,0.9)$ $((711.520,750.240,827.680,866.400 ; 1,1)$ $(730.880,769.600,808.320,847.040 ; 0.9,0.9))$ ((711.520, 750.240, 827.680, 866.400:1, 1), $(730.880,769.600,808.320,847.040 ; 0.9,0.9)$ ((711.520, 750.240, 827.680, 866.400;1, 1), $(730.880,769.600,808.320,847.040 ; 0.9,0.9)$ ((711.520, 750.240, 827.680, 866.400;1, 1 ), $(730.880,769.600,808.320,847.040 ; 0.9,0.9)$ ((711.520, 750.240, 827.680, 866.400;1, 1) $(730.880,769.600,808.320,847.040 ; 0.9,0.9)$ ((711.520, 750.240, 827.680, 866.400;1, 1), $(730.880,769.600,808.320,847.040 ; 0.9,0.9)$ $((711.520,750.240,827.680,866.400: 1,9)$

\section{Accumulated Depreciation}

$(711.520,750.240,827.680,866.400 ; 1,1)$,
$((1.461 .760,1.539 .400,1.65 .360,1.732 .800 ; 1,1)$, $(2.134 .560,2.250 .720,2.483 .040,2.599 .200 ; 1,1)$, (2.192.640, 2.308.800, 2.424.960, 2.541.120;0.9, 0.9) ((2.846.080, 3.000.960, 3.310.720, 3.465.600;1, 1), (1) (3.557.600, 3.751.200, 4.138.400, 4.332.000;1, 1), $(3.654 .400,3.848 .000,4.041 .600,4.235 .200 ; 0.9,0.9)$ ((4.269.120, 4.501.440, 4.966.080, 5.198.400;1, 1), (4.385.280, 4.617.600, 4.849.920, 5.082.240;0.9, 0.9) ((4.980.640, 5.251.680, 5.793.760, 6.064.800;1, 1), (6.160, 5.387.200, 5.658.240, 5.929.280;0.9, 0.9) $(5.692 .160,6.001 .920,6.621 .440,6.931 .200: 1,1)$ $(5.847 .040,6.156 .800,6.466 .560,6.776 .320 \cdot 0.9,0.9))$

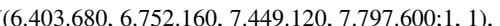
$(6.577 .920,6.926 .400,7.274 .880,7.623 .360 \cdot 0.9,0.9))$ $((7.115 .200,7.502 .400 .8 .276 .800,8.664 .000: 1,1)$ $(7.308 .800,7.696 .000,8.083 .200,8.470 .400 \cdot 0.9,0.9)$ $((7.826 .720,8.252 .640,9.104 .480,9.530 .400 ; 1,1)$ $(8.039 .680,8.465 .600,8.891 .520,9.317 .440 ; 0.9,0.9))$ ((8.538.240,9.002 880,9.932.160, 10.396.800:1 1), $(8.770 .560,9.235 .200,9.699 .840,10.164 .480 ; 0.9,0.9))$ $((9.249 .760,9.753 .120,10.759 .840,11.263 .200 \cdot 1,1)$ $(9.501 .440,10.004 .800,10.508 .160,11.011 .520 .0 .9$, 0.98$)$ (9.961.280, 10.503.360, 11.587.520, 12.129.600;1, 1), $(10.232 .320,10.774 .400,11.316 .480,11.858 .560 \cdot 0.9$,

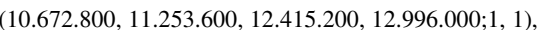
$(10.963 .200,11.544 .000,12.124 .800,12.705 .600 ; 0.9,0.9)$ ((11.384.320, 12.003.840, 13.242.880, 13.862.400;1. 1) $(11.694 .080,12.313 .600,12.933 .120,13.552 .640 ; 0.9,0.9)$ ((12.095.840, 12.754.080, 14.070.560, 14.728.800;1, 1), $(12.424 .960,13.083 .200,13.741 .440,14.399 .680 ; 0.9,0.9)$ ((12.807.360, 13.504.320, 14.898.240, 15.595.200;1, 1) $(13.155 .840,13.852 .800,14.549 .760,15.246 .720 ; 0.9,0.9)$ (13.518.880, 14.254.560, 15.725.920, 16.461.600;1, 1), (3.886.720, 14.622.400, 15.358.080, 16.093.760;0.9, 0.9) (14.230.400, 15.004.800, $16.553 .600,17.328 .000 \cdot 1,1)$, , 15.392.000, 16.166.400, 16.940.800;0.9, 0. (14.941.920, 15.755.040, 17.381.280, 18.194.400;1, 1), (15.348.480, 16.161.600, 16.974.720, 17.787.840;0.9, (15.653.440, 16.505.280, 18.208.960, 19.060.800;1, 1), (6.931.200, $17.783 .040,18.634 .880 ; 0.9,0$. (16.364.960, 17.255.520, 19.036.640, 19.927.200;1, 1) (ת. (1).800, $18.591 .360,19.481 .920 ; 0.9,0.9)$ ((17.076.480, 18.005.760, 19.864.320, 20.793.600:1, 1), (19.5) , ((17.788.000, 18.756.000, 20.692.000, 21.660.000;1, 1)

\section{Book Value at End of Year}

((21.133.600, 21.672.320, 22.749.760, 23.288.480;1, 1), (3) ((20.555.200, 20.844.640, 21.999.520, 22.576.960;1, 1), (19.400.800, 20.016.960, 21249.280, 21.865.440:1,1), ((19.700.880, 20.016.960, 21.249.280, 21.865.440;1,1), (18.534.400, $19.189 .280,20.499 .040,21.153 .920 .1,1)$ (18.561.400, 19.1816.280, 20.491.040, 21.153.920,1, 1),

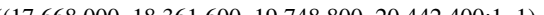
$(18.014 .000,18.708 .400,19.402 .000,20.095 .600 ; 0.9,0.9))$ ((16.801.600, 17.533.920, 18.998.560, 19.730.880:1, 1), $(17.167 .760,17.500 .080,18.632400,19.364 .720 ; 0.9,0.9)$ $((15.935 .200,16706.240,18.248 .320,19.019 .360 .1,1)$ $(16.320 .720,17.091 .760,17.862800,18.633 .840 ; 0.9,0.9))$ ((15.068.800, 15.878.560, 17.498.080, 18.307.840;1, 1), $(15.473 .680,16.283 .440,17.093 .200,17.902 .960 ; 0.9,0.9)$ $((14.202 .400,15.050 .880,16.747 .840,17.596 .320 ; 1,1)$ $(14.626 .640,15.475 .120,16.323 .600,17.172080 \cdot 0.9), 0.9)$ $((13.336 .000,14.223 .200,15.997 .600,16.884 .800 ; 1,1)$ $(13.779 .600,14.666 .800,15.554 .000,16.441 .200 \cdot 0.9,0.9))$ ((12.469.600, 13.395.520, 15.247.360, 16.173.280:1,1), $(12.932 .560,13.858 .480,14.784 .400,15.710 .320 ; 0.9,0.9)$ $((11.603 .200,12.567 .840,14.497 .120,15.461 .760 \cdot 1,1)$, $(12.085 .520,13.050 .160,14.014 .800,14.979 .440 ; 0.9,0.9)$ $((10.736 .800,11.740 .160,13.746 .880,14.750 .240 ; 1,1)$ $(11.238 .480,12.241 .840,13.245 .200,14.248 .560 ; 0.9,0.9))$ ( (9.870.400, 10.912.480, 12.996.640, 14.038.720;1, 1). $(10.391 .440,11.433 .520,12.475 .600,13.517 .680 ; 0.9,0.9))$ $((9.004 .000,10.084 .800,12.246 .400,13.327 .200 ; 1,1)$ $(9.544 .400,10.625 .200,11.706 .000,12.786 .800 ; 0.9,0.9)$ $((8.137 .600,9.257 .120,11.496 .160,12.615 .680 \cdot 1.1)$, $(8.697 .360,9.816 .880,10.936 .400,12.055 .920 ; 0.9,0.9))$ ((7.271.200, 8.429.440, 10.745.920, 11.904.160;1, 1), $(7.850 .320,9.008 .560,10.166 .800,11.325 .040 ; 0.9,0.9)$ ((6.404.800, 7.601.760, 9.995.680, 11.192.640;1, 1), $(7.003 .280,8.200 .240,9.397 .200,10.594 .160 ; 0.9,0.9))$ ((5.538.400, 6.774.080, 9.245.440, 10.481.120;1, 1), $(6.156 .240,7.391 .920,8.627 .600,9.863 .280 ; 0.9,0.9))$ ((4.672.000, 5.946.400, 8.495.200, 9.769.600;1, 1), ( ((3.805.600, 5.118.720, 7.744.960, 9.058.080;1, 1), $5.775 .280,7.088 .400,8.401 .520 ; 0.9,0.9)$ ((2.939.200, 4.291.040, 6.994.720, 8.346.560;1, 1), (1) (2.072.800, 3.463.360, 6.244.480, 7.635.040;1, 1), $((1.206 .400,2.635 .680,5.494 .240,6.923 .520 ; 1,1)$ $((340.000,1.808 .000,4.744 .000,6.212 .000 \cdot 1,1)$ 
Table 5: Details of breakdown for classic Double Declining Balance Depreciation method.

\begin{tabular}{|c|c|c|c|c|}
\hline Year & Book Value at Beginning of Year & Depreciation Expense & Accumulated Depreciation & Book Value at End of Year \\
\hline 1 & 23.000 .000 & 1.840 .000 & 1.840 .000 & 21.160 .000 \\
\hline 2 & 21.160 .000 & 1.692 .800 & 3.532 .800 & 19.467 .200 \\
\hline 3 & 19.467.200 & 1.557 .376 & 5.090 .176 & 17.909 .824 \\
\hline 4 & 17.909 .824 & 1.432 .786 & 6.522 .962 & 16.477 .038 \\
\hline 5 & 16.477 .038 & 1.318 .163 & 7.841 .125 & 15.158 .875 \\
\hline 6 & 15.158 .875 & 1.212 .710 & 9.053 .835 & 13.946 .165 \\
\hline 7 & 13.946 .165 & 1.115 .693 & 10.169 .528 & 12.830 .472 \\
\hline 8 & 12.830 .472 & 1.026 .438 & 11.195 .966 & 11.804 .034 \\
\hline 9 & 11.804 .034 & 944.323 & 12.140 .289 & 10.859 .711 \\
\hline 10 & 10.859 .711 & 868.777 & 13.009 .066 & 9.990 .934 \\
\hline 11 & 9.990 .934 & 799.275 & 13.808 .340 & 9.191 .660 \\
\hline 12 & 9.191 .660 & 735.333 & 14.543 .673 & 8.456 .327 \\
\hline 13 & 8.456 .327 & 676.506 & 15.220 .179 & 7.779 .821 \\
\hline 14 & 7.779 .821 & 622.386 & 15.842 .565 & 7.157 .435 \\
\hline 15 & 7.157.435 & 572.595 & 16.415 .160 & 6.584 .840 \\
\hline 16 & 6.584 .840 & 526.787 & 16.941 .947 & 6.058 .053 \\
\hline 17 & 6.058 .053 & 484.644 & 17.426 .591 & 5.573 .409 \\
\hline 18 & 5.573 .409 & 445.873 & 17.872 .464 & 5.127 .536 \\
\hline 19 & 5.127 .536 & 410.203 & 18.282 .667 & 4.717 .333 \\
\hline 20 & 4.717 .333 & 377.387 & 18.660 .053 & 4.339 .947 \\
\hline 21 & 4.339 .947 & 347.196 & 19.007.249 & 3.992 .751 \\
\hline 22 & 3.992 .751 & 319.420 & 19.326 .669 & 3.673 .331 \\
\hline 23 & 3.673 .331 & 293.866 & 19.620 .536 & 3.379 .464 \\
\hline 24 & 3.379 .464 & 103.464 & 19.724 .000 & 3.276 .000 \\
\hline 25 & - & - & - & - \\
\hline
\end{tabular}


Table 6: Details of breakdown for triangular interval type-2 fuzzy for Double Declining Balance Depreciation method.

\begin{tabular}{|c|c|c|c|c|}
\hline Year & Book Value at Beginning of Year & Depreciation Expense & Accumulated Depreciation & Book Value at End of Year \\
\hline 1 & $\begin{array}{l}((22.000 .000,23.000 .000,24.000 .000 ; 1) \\
(22.250 .000,23.000 .000,23.750 .000 ; 0.9))\end{array}$ & $\begin{array}{l}((1.760 .000,1.840 .000,1.920 .000 ; 1), \\
(1.780 .000,1.840 .000,1.900 .000 ; 0.9))\end{array}$ & $\begin{array}{l}((1.760 .000,1.840 .000,1.920 .000 ; 1), \\
(1.780 .000,1.840 .000,1.900 .000 ; 0.9))\end{array}$ & $\begin{array}{l}((20.080 .000,21.160 .000,22.240 .000 ; 1), \\
(20.350 .000,21.160 .000,21.970 .000 ; 0,9))\end{array}$ \\
\hline 2 & $\begin{array}{l}((20.080 .000,21.160 .000,22.240 .000 ; 1) \\
(20.350 .000,21.160 .000,21.970 .000 ; 0,9))\end{array}$ & $\begin{array}{l}((1.606 .400,1.692 .800,1.779 .200 ; 1), \\
(1.628 .000,1.692 .800,1.757 .600 ; 0,9))\end{array}$ & $\begin{array}{l}((3.366 .400,3.532 .800,3.699 .200 ; 1), \\
(3.408 .000,3.532 .800,3.657 .600 ; 0,9))\end{array}$ & $\begin{array}{l}(20.350 .000,21.160 .000,21.970 .000 ; 0,9)) \\
((18.300 .800,19.467 .200,20.633 .600 ; 1), \\
(18.592 .400,19.467 .200,20.342 .000 ; 0,9))\end{array}$ \\
\hline 3 & $\begin{array}{l}((18.300 .800,19.467 .200,20.633 .600 ; 1) \\
(18.592 .400,19.467 .200,20.342 .000 ; 0,9))\end{array}$ & $\begin{array}{l}((1.464 .064,1.557 .376,1.650 .688 ; 1) \\
(1.487 .392,1.557 .376,1.627 .360 ; 0,9))\end{array}$ & $\begin{array}{l}((4.830 .464,5.090 .176,5.349 .888 ; 1) \\
(4.895 .392,5.090 .176,5.284 .960 ; 0,9))\end{array}$ & $\begin{array}{l}((16.650 .112,17.909 .824,19.169 .536 ; 1), \\
(16.965 .040,17.909 .824,18.854 .608 ; 0,9))\end{array}$ \\
\hline 4 & $\begin{array}{l}((16.650 .112,17.909 .824,19.169 .536 ; 1) \\
(16.965 .040,17.909 .824,18.854 .608 ; 0,9))\end{array}$ & $\begin{array}{l}((1.332 .009,1.432 .786,1.533 .563 ; 1), \\
(1.357 .203,1.432 .786,1.508 .369 ; 0,9))\end{array}$ & $\begin{array}{l}((6.162 .473,6.522 .962,6.883 .451 ; 1), \\
(6.252 .595,6.522 .962,6.793 .329 ; 0,9))\end{array}$ & $\begin{array}{l}((15.116 .549,16.477 .038,17.837 .527 ; 1), \\
(15.456 .671,16.477 .038,17.497 .405 ; 0,9))\end{array}$ \\
\hline 5 & $\begin{array}{l}((15.116 .549,16.477 .038,17.837 .527 ; 1) \\
(15.456 .671,16.477 .038,17.497 .405 ; 0,9))\end{array}$ & $\begin{array}{l}((1.209 .324,1.318 .163,1.427 .002 ; 1) \\
(1.236 .534,1.318 .163,1.399 .792 ; 0,9))\end{array}$ & $\begin{array}{l}((7.371 .797,7.841 .125,8.310 .453 ; 1), \\
(7.489 .129,7.841 .125,8.193 .121 ; 0,9))\end{array}$ & $\begin{array}{l}((13.689 .547,15.158 .875,16.628 .203 ; 1), \\
(14.056 .879,15.158 .875,16.260 .871 ; 0,9))\end{array}$ \\
\hline 6 & $\begin{array}{l}((13.689 .547,15.158 .875,16.628 .203 ; 1), \\
(14.056 .879,15.158 .875,16.260 .871 ; 0,9))\end{array}$ & $\begin{array}{l}((1.095 .164,1.212 .710,1.330 .256 ; 1), \\
(1.124 .550,1.212 .710,1.300 .870 ; 0,9))\end{array}$ & $\begin{array}{l}((8.466 .961,9.053 .835,9.640 .709 ; 1), \\
(8.613 .679,9.053 .835,9.493 .991 ; 0,9))\end{array}$ & $\begin{array}{l}((12.359 .291,13.946 .165,15.533 .039 ; 1), \\
(12.756 .009,13.946 .165,15.136 .321 ; 0,9))\end{array}$ \\
\hline 7 & $\begin{array}{l}((12.359 .291,13.946 .165,15.533 .039 ; 1) \\
(12.756 .009,13.946 .165,15.136 .321 ; 0,9))\end{array}$ & $\begin{array}{l}((988.743,1.115 .693,1.242 .643 ; 1), \\
(1.020 .481,1.115 .693,1.210 .906 ; 0,9))\end{array}$ & $\begin{array}{l}((9.455 .704,10.169 .528,10.883 .352 ; 1) \\
(9.634 .160,10.169 .528,10.704 .896 ; 0,9))\end{array}$ & $\begin{array}{l}((11.116 .648,12.830 .472,14.544 .296 ; 1), \\
(11.545 .104,12.830 .472,14.115 .840 ; 0,9))\end{array}$ \\
\hline 8 & $\begin{array}{l}((11.116 .648,12.830 .472,14.544 .296 ; 1) \\
(11.545 .104,12.830 .472,14.115 .840 ; 0,9))\end{array}$ & $\begin{array}{l}((889.332,1.026 .438,1.163 .544 ; 1) \\
(923.608,1.026 .438,1.129 .267 ; 0,9))\end{array}$ & $\begin{array}{l}((10.345 .036,11.195 .966,12.046 .896 ; 1) \\
(10.557 .768,11.195 .966,11.834 .164 ; 0,9))\end{array}$ & $\begin{array}{l}((9.953 .104,11.804 .034,13.654 .964 ; 1), \\
(10.415 .836,11.804 .034,13.192 .232 ; 0,9))\end{array}$ \\
\hline 9 & $\begin{array}{l}((9.953 .104,11.804 .034,13.654 .964 ; 1) \\
(10.415 .836,11.804 .034,13.192 .232 ; 0,9))\end{array}$ & $\begin{array}{l}((796.248,944.323,1.092 .397 ; 1) \\
(833.267,944.323,1.055 .379 ; 0,9))\end{array}$ & $\begin{array}{l}((11.141 .284,12.140 .289,13.139 .293 ; 1) \\
(11.391 .035,12.140 .289,12.889 .542 ; 0,9))\end{array}$ & $\begin{array}{l}((8.860 .707,10.859 .711,12.858 .716 ; 1), \\
(9.360 .458,10.859 .711,12.358 .965 ; 0,9))\end{array}$ \\
\hline 10 & $\begin{array}{l}((8.860 .707,10.859 .711,12.858 .716 ; 1) \\
(9.360 .458,10.859 .711,12.358 .965 ; 0,9))\end{array}$ & $\begin{array}{l}((708.857,868.777,1.028 .697 ; 1), \\
(748.837,868.777,988.717 ; 0,9))\end{array}$ & $\begin{array}{l}((11.850 .141,13.009 .066,14.167 .991 ; 1) \\
(12.139 .872,13.009 .066,13.878 .259 ; 0,9))\end{array}$ & $\begin{array}{l}((7.832 .009,9.990 .934,12.149 .859 ; 1)) \\
(8.371 .741,9.990 .934,11.610 .128 ; 0,9))\end{array}$ \\
\hline 11 & $\begin{array}{l}((7.832 .009,9.990 .934,12.149 .859 ; 1) \\
(8.371 .741,9.990 .934,11.610 .128 ; 0,9))\end{array}$ & $\begin{array}{l}((626.561,799.275,971.989 ; 1) \\
(669.739,799.275,928.810 ; 0,9))\end{array}$ & $\begin{array}{l}((12.476 .701,13.808 .340,15.139 .979 ; 1), \\
(12.809 .611,13.808 .340,14.807 .070 ; 0,9))\end{array}$ & $\begin{array}{l}((6.860 .021,9.191 .660,11.523 .299 ; 1) \\
(7.442 .930,9.191 .660,10.940 .389 ; 0,9))\end{array}$ \\
\hline 12 & $\begin{array}{l}((6.860 .021,9.191 .660,11.523 .299 ; 1) \\
(7.442 .930,9.191 .660,10.940 .389 ; 0,9))\end{array}$ & $\begin{array}{l}((548.802,735.333,921.864 ; 1), \\
(595.434,735.333,875.231 ; 0,9))\end{array}$ & $\begin{array}{l}((13.025 .503,14.543 .673,16.061 .843 ; 1) \\
(13.405 .045,14.543 .673,15.682 .301 ; 0,9))\end{array}$ & $\begin{array}{l}((5.938 .157,8.456 .327,10.974 .497 ; 1), \\
(6.567 .699,8.456 .327,10.344 .955 ; 0,9))\end{array}$ \\
\hline 13 & $\begin{array}{l}((5.938 .157,8.456 .327,10.974 .497 ; 1) \\
(6.567 .699,8.456 .327,10.344 .955 ; 0,9))\end{array}$ & $\begin{array}{l}((475.053,676.506,877.960 ; 1) \\
(525.416,676.506,827.596 ; 0,9))\end{array}$ & $\begin{array}{l}((13.500 .556,15.220 .179,16.939 .803 ; 1) \\
(13.930 .461,15.220 .179,16.509 .897 ; 0,9))\end{array}$ & $\begin{array}{l}((5.060 .197,7.779 .821,10.499 .444 ; 1) \\
,(5.740 .103,7.779 .821,9.819 .539 ; 0,9))\end{array}$ \\
\hline 14 & $\begin{array}{l}((5.060 .197,7.779 .821,10.499 .444 ; 1) \\
(5.740 .103,7.779 .821,9.819 .539 ; 0,9))\end{array}$ & $\begin{array}{l}((404.816,622.386,839.956 ; 1) \\
(459.208,622.386,785.563 ; 0,9))\end{array}$ & $\begin{array}{l}((13.905 .371,15.842 .565,17.779 .759 ; 1) \\
(14.389 .670,15.842 .565,17.295 .460 ; 0,9))\end{array}$ & $\begin{array}{l}((4.220 .241,7.157 .435,10.094 .629 ; 1) \\
,(4.954 .540,7.157 .435,9.360 .330 ; 0,9))\end{array}$ \\
\hline 15 & $\begin{array}{l}((4.220 .241,7.157 .435,10.094 .629 ; 1), \\
(4.954 .540,7.157 .435,9.360 .330 ; 0,9))\end{array}$ & $\begin{array}{l}((337.619,572.595,807.570 ; 1), \\
(396.363,572.595,748.826 ; 0,9))\end{array}$ & $\begin{array}{l}((14.242 .991,16.415 .160,18.587 .329 ; 1) \\
(14.786 .033,16.415 .160,18.044 .287 ; 0,9))\end{array}$ & $\begin{array}{l}((3.412 .671,6.584 .840,9.757 .009 ; 1) \\
(4.205 .713,6.584 .840,8.963 .967 ; 0,9))\end{array}$ \\
\hline 16 & $\begin{array}{l}((3.412 .671,6.584 .840,9.757 .009 ; 1), \\
(4.205 .713,6.584 .840,8.963 .967 ; 0,9))\end{array}$ & $\begin{array}{l}((273.014,526.787,780.561 ; 1), \\
(336.457,526.787,717.117 ; 0,9))\end{array}$ & $\begin{array}{l}((14.516 .004,16.941 .947,19.367 .890 ; 1) \\
(15.122 .490,16.941 .947,18.761 .404 ; 0,9))\end{array}$ & $\begin{array}{l}((2.632 .110,6.058 .053,9.483 .996 ; 1) \\
(3.488 .596,6.058 .053,8.627 .510 ; 0,9))\end{array}$ \\
\hline 17 & $\begin{array}{l}((2.632 .110,6.058 .053,9.483 .996 ; 1) \\
(3.488 .596,6.058 .053,8.627 .510 ; 0,9))\end{array}$ & $\begin{array}{l}((210.569,484.644,758.720 ; 1) \\
(279.088,484.644,690.201 ; 0,9))\end{array}$ & $\begin{array}{l}((14.726 .573,17.426 .591,20.126 .609 ; 1) \\
(15.401 .578,17.426 .591,19.451 .605 ; 0,9))\end{array}$ & $\begin{array}{l}((1.873 .391,5.573 .409,9.273 .427 ; 1) \\
(2.798 .395,5.573 .409,8.348 .422 ; 0,9))\end{array}$ \\
\hline 18 & $\begin{array}{l}((1.873 .391,5.573 .409,9.273 .427 ; 1) \\
(2.798 .395,5.573 .409,8.348 .422 ; 0,9))\end{array}$ & $\begin{array}{l}((149.871,445.873,741.874 ; 1), \\
(223.872,445.873,667.874 ; 0,9))\end{array}$ & $\begin{array}{l}((14.876 .444,17.872 .464,20.868 .483 ; 1) \\
(15.625 .449,17.872 .464,20.119 .479 ; 0,9))\end{array}$ & $\begin{array}{l}((1.131 .517,5.127 .536,9.123 .556 ; 1) \\
(2.130 .521,5.127 .536,8.124 .551 ; 0,9))\end{array}$ \\
\hline 19 & $\begin{array}{l}((1.131 .517,5.127 .536,9.123 .556 ; 1) \\
(2.130 .521,5.127 .536,8.124 .551 ; 0,9))\end{array}$ & $\begin{array}{l}((90.521,410.203,729.884 ; 1) \\
(170.442,410.203,649.964 ; 0,9))\end{array}$ & $\begin{array}{l}((14.966 .966,18.282 .667,21.598 .368 ; 1) \\
(15.795 .891,18.282 .667,20.769 .443 ; 0,9))\end{array}$ & $\begin{array}{l}((401.632,4.717 .333,9.033 .034 ; 1) \\
(1.480 .557,4.717 .333,7.954 .109 ; 0,9))\end{array}$ \\
\hline 20 & $\begin{array}{l}((401.632,4.717 .333,9.033 .034 ; 1) \\
(1.480 .557,4.717 .333,7.954 .109 ; 0,9))\end{array}$ & $\begin{array}{l}((32.131,377.387,722.643 ; 1), \\
(118.445,377.387,636.329 ; 0,9))\end{array}$ & $\begin{array}{l}((14.999 .096,18.660 .053,22.321 .011 ; 1) \\
(15.914 .336,18.660 .053,21.405 .771 ; 0,9))\end{array}$ & $\begin{array}{l}((-321.011,4.339 .947,9.000 .904 ; 1) \\
(844.229,4.339 .947,7.835 .664 ; 0,9))\end{array}$ \\
\hline 21 & $\begin{array}{l}((-321.011,4.339 .947,9.000 .904 ; 1) \\
(844.229,4.339 .947,7.835 .664 ; 0,9))\end{array}$ & $\begin{array}{l}((-25.681,347.196,720.072 ; 1) \\
(67.538,347.196,626.853 ; 0,9))\end{array}$ & $\begin{array}{l}((14.973 .415,19.007 .249,23.041 .083 ; 1) \\
(15.981 .874,19.007 .249,22.032 .624 ; 0,9))\end{array}$ & $\begin{array}{l}((-1.041 .083,3.992 .751,9.026 .585 ; 1) \\
(217.376,3.992 .751,7.768 .126 ; 0,9))\end{array}$ \\
\hline 22 & $\begin{array}{l}((-1.041 .083,3.992 .751,9.026 .585 ; 1), \\
(217.376,3.992 .751,7.768 .126 ; 0,9))\end{array}$ & $\begin{array}{l}((-83.287,319.420,722.127 ; 1) \\
(17.390,319.420,621.450 ; 0,9))\end{array}$ & $\begin{array}{l}((14.890 .129,19.326 .669,23.763 .210 ; 1) \\
(15.999 .264,19.326 .669,22.654 .075 ; 0,9))\end{array}$ & $\begin{array}{l}((-1.763 .210,3.673 .331,9.109 .871 ; 1) \\
(-404.075,3.673 .331,7.750 .736 ; 0,9))\end{array}$ \\
\hline 23 & $\begin{array}{l}((-1.763 .210,3.673 .331,9.109 .871 ; 1) \\
(-404.075,3.673 .331,7.750 .736 ; 0,9))\end{array}$ & $\begin{array}{l}((-141.057,293.866,728.790 ; 1) \\
(-32.326,293.866,620.059 ; 0,9))\end{array}$ & $\begin{array}{l}((14.749 .072,19.620 .536,24.491 .999 ; 1) \\
(15.966 .938,19.620 .536,23.274 .133 ; 0,9))\end{array}$ & $\begin{array}{l}((-2.491 .999,3.379 .464,9.250 .928 ; 1) \\
(-1.024 .133,3.379 .464,7.783 .062 ; 0,9))\end{array}$ \\
\hline 24 & $\begin{array}{l}((-2.491 .999,3.379 .464,9.250 .928 ; 1) \\
(-1.024 .133,3.379 .464,7.783 .062 ; 0.9))\end{array}$ & $\begin{array}{l}((-6.703 .999,103.464,6.910 .928 ; 1) \\
(-5.002 .133,103.464,5.209 .062 ; 0.9))\end{array}$ & $\begin{array}{l}((8.045 .073,19.724 .000,31.402 .927 ; 1) \\
(10.964 .805,19.724 .000,28.483 .195 ; 0.9))\end{array}$ & $\begin{array}{l}((2.340 .000,3.276 .000,4.212 .000 ; 1), \\
(2.574 .000,3.276 .000,3.978 .000 ; 0.9))\end{array}$ \\
\hline 25 & - & - & - & - \\
\hline
\end{tabular}


Table 7: Details of breakdown for trapezoidal interval type-2 fuzzy for Double Declining Balance Depreciation method.

Year Book Value at Beginning of Year

$((22.000 .000,22.500 .000,23.500 .000,24.000 .000 ; 1,1)$

(20.250.00, 22.750.000, 23.250.000, 23.750.000,0,9, , ,

(20.080.00, 20.620.000, 21.700.000, 22.240.000,1, 1)

(18.300.800, 18.884.000, 20.050.400, 20.633.000;1, 1)

(18.502.800, 18.884.000, 20.050.400, 20.633.600,1, 1),

((16.650.112, 17.279.968, 18.539.680, 19.169.536:1, 1),

(16.665.040, 17.594.896, 18.224.752, $18.854 .608,0,9,0,0)$

$((15.116 .549,15.796 .794,17.157 .283,17.837 .527,1,1)$

(15.456.671, 16.136.916, 16.817.160,17.497.405:0,9,0,9)

(13.689.547, 14.424.211, 15.893.539, 16.628.203,1,1),

$6 \quad(14.056 .579,14.791543,15.526207,16.6280 .871 \cdot 0.9,0,0)$

((12.359.291, 13.152.728, 14.739.602, 15.533.039:1, 1),

$(12.756 .009,13.549 .746,14.342 .684,15.136 .0321 \cdot 0.9,0.9))$

((11.116.648, 11.973.560, 13.687.384, 14.544.296;1, 1),

(11.545.104, 12.402.016, 13.258.928, 14.115.840:0.9,0.9)

$((9.953 .104,10.878 .569,12.729 .499,13.654 .964: 1,1)$,

$(10.415 .836,11.341 .302,12.266 .767,13.192 .232 \cdot 0,9,0,9))$

$((8.860 .707,9.860 .209,11.859 .214,12.858 .716 ; 1,1)$

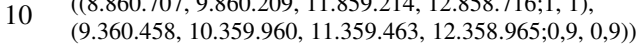

$((7.832 .009,8.911 .472,11.070 .397,12.149 .859 ; 1,1)$,

(8.371.741, 9.451.203, 10.530.666, 11.610.128;0,9, 0,9$)$

((6.860.021, 8.025.840, 10.357.479, 11.523.299;1, 1),

$(7.442 .930,8.608 .750,9.774 .569,10.940 .389 ; 0,9,0,9))$

((5.938.157, 7.197.242, 9.715.412, 10.974.497;1, 1),

$(6.567 .699,7.826 .784,9.085 .869,10.344 .955 ; 0,9,0,9)$

( $(5.060 .197,6.420 .009,9.139 .633,10.499 .444 ; 1,1)$,

$(5.740 .103,7.099 .915,8.459 .727,9.819 .539 ; 0,9,0,9))$

$((4.220 .241,5.688 .838,8.626 .032,10.094 .629 ; 1,1)$,
$(4.954 .540,6.423 .137,7.891 .734,9.360 .330 \cdot 0.9,0.9))$

$((3.412 .671,4.998 .756,8.170 .925,9.757 .009 \cdot 1,1)$

$(4.205 .713,5.791 .798,7.377 .883,8.963 .967 ; 0,9,0,9))$

((2.632.110, 4.345.082, 7.771.024, 9.483.996;1, 1)

$(3.488 .596,5.201 .567,6.914 .539,8.627 .510 ; 0,9,0,9))$

((1.873.391, 3.723.400, 7.423.418, 9.273.427;1, 1),

$(2.798 .395,4.648 .404,6.498 .413,8.348 .422 ; 0,9,0,9))$

((1.131.517, 3.129.526, 7.125.546, 9.123.556;1, 1),

$(2.130 .521,4.128 .531,6.126 .541,8.124 .551 ; 0,9,0,9))$

((401.632, 2.559.483, 6.875.184, 9.033.034;1, 1)

$(1.480 .557,3.638 .408,5.796 .258,7.954 .109 ; 0,9,0,9)$

((-321.011, 2.009.468, 6.670.425, 9.000.904:1, 1),

$(844.229,3.174 .707,5.505 .186,7.835 .664 ; 0,9,0,9))$

$((-1.041 .083,1.475 .834,6.509 .668,9.026 .585 ; 1,1)$

$(217.376,2.734 .292,5.251 .209,7.768 .126 ; 0,9,0,9))$

((-1.763.210, 955.061, 6.391.601, 9.109.871;1, 1),

$(-404.075,2.314 .196,5.032 .466,7.750 .736 ; 0,9,0,9)$

$((-2.491 .999,443.732,6.315 .196,9.250 .928 ; 1,1)$

$(-1.024 .133,1.911 .598,4.847 .330,7.783 .062 ; 0.9,0.9))$

\section{Depreciation Expense}

$((1.760 .000,1.800 .000,1.880 .000,1.920 .000 ; 1,1)$

(1.606.400, 1.649.600, 1.736.000, $1.779 .200 ; 1,1)$

(1.606.400, 1.649.600, 1.736.000, 1.779.200,1, 1),

( $(1.464 .064,1.510 .720,1.604 .032,1.650 .688 ; 1,1)$

(.534.048, 1.580.704, $1.627 .360 \cdot 0.9,0.9)$

$(1.332 .009,1.382 .397,1.483 .174,1.533 .563 ; 1,1)$

)

(1.236.534, 1.260.953,1.375.583, $1.427 .002 ; 1,1)$

(1.095.164, 1.153.937, 1.271.483,1.330.256;1,1),

(1.124.550, $1.183 .323,1.242 .097,1.300 .870,0.9,0,9))$

(1.088.743, 1.052 218, 1.179.168, 1.242.643:1, 1),

(1.020.481, 1.083.956, 1.147.431, 1.210.906-0.9,0,9)

((889.332, 957.885, $1.094 .991,1.163 .544 \cdot 1,1)$,

$(923.608,992.161,1.060 .714,1.129 .267 ; 0,9,0,9))$

(796.248,870.286, 1.018.360, $1.092397: 1,1)$

$(833.267,907.304,981.341,1.055 .379 \cdot 0.9,0.9))$

((708.857, 788.817, 948.737, 1.028.697:1, 1),

$(748.837,828.797,908.757,988.71770,9,0,9)$

$((626.561,712.918,885.632,971.989 ; 1,1)$

$(669.739,756.096,842.453,928.810 ; 0,9,0,9))$

(548.802, 642.067, 828.598, 921.864:1,1), (19., )

$(595.434,688.700,781.966,875.231 ; 0,9,0,9)$

$((475.053,575.779,777.233,877.960 ; 1,1)$

$(525.416,626.143,726.870,827.596 ; 0,9,0,9))$

((404.816, 513.601, 731.171, 839.956;1, 1),

$(459.208,567.993,676.778,785.563 ; 0,9,0,9))$

$((337.619,455.107,690.083,807.570 ; 1,1)$

$(396.363,513.851,631.339,748.826 ; 0,9,0,9)$

((273.014, 399.900, 653.674, 780.561:1, 1),

$(336.457,463.344,590.231,717.117 ; 0,9,0,9)$

((210.569, 347.607, 621.682, 758.720;1, 1),

$(279.088,416.125,553.163,690.201 ; 0,9,0,9))$

((149.871, 297.872, 593.873, 741.874;1, 1),

$(223.872,371.872,519.873,667.874 ; 0,9,0,9)$

( $(90.521,250.362,570.044,729.884 ; 1,1)$,

$(170.442,330.282,490.123,649.964 ; 0,9,0,9))$

((32.131, 204.759, 550.015, 722.643;1, 1),

$(118.445,291.073,463.701,636.329 ; 0,9,0,9))$

((-25.681, 160.757, 533.634, 720.072;1, 1),

$(67.538,253.977,440.415,626.853 ; 0,9,0,9))$

$((-83.287,118.067,520.773,722.127 ; 1,1)$,

$(17.390,218.743,420.097,621.450 ; 0,9,0,9))$

$((-141.057,76.405,511.328,728.790 ; 1,1)$

$(-32.326,185.136,402.597,620.059 ; 0,9,0,9))$

$((-6.703 .999,-3.300 .268,3.507 .196,6.910 .928 ; 1,1)$

$(-5.002 .133,-1.598 .402,1.805 .330,5.209 .062 ; 0.9,0.9)$

\section{Accumulated Depreciation}

((1.760.000, 1.800.000, 1.880.000, 1.920.000;1, 1),

(3.9.

(3.408.400, 3.449.600, 3.616.00, 3.659.200,1, 1 ,

(4.830.464, $4.960 .320,5220.032,5.349 .888 \cdot 1,1)$

(4.895.392, 5.025.248, 5.22.032, 5.349.888,1, 1 ,

((6.162.473, 6.342.717, 6.703.206, 6.883.451:1,1),

(6.252.595, 6.432.840, 6.613.084, 6.793.329,1, 1$)$,

(7.371.797, 7.606.461, 8.075.789,8.310.453:1,1),

(7.489.129, 7.723.793, $7.958 .457,8.193 .121 ; 0.9,0,9)$

$((8.466 .961,8.760 .398,9.347 .272,9.640 .709 ; 1,1)$

(8.613.679, 8.907.116, 9.200.554, $9.493 .991 ; 0.9,0,9)$

$((9.455 .704,9.812 .616,10.526 .440,10.883 .352 .1,1)$

$(9.634 .160,9.991 .072,10.347 .984,10.704 .896 ; 0,9,0,9))$

$((10.345 .036,10.770 .501,11.621 .431,12.046 .896 \cdot 1,1)$,

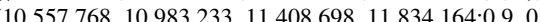

$((11.141 .284,11.640 .786,12.639 .791,13.139 .293: 1,1)$

$(11.391 .035,11.890 .537,12.390 .040,12.889 .542 ; 0,9,0,9)$

((11.850.141, 12.429.603, 13.588.528, 14.167.991;1, 1),

$(12.139 .872,12.719 .334,13.298 .797,13.878 .259 ; 0,9,0,9))$

$(12.476 .701,13.142 .521,14.474 .160,15.139 .979 ; 1,1)$,
$(12.809 .611,13.475 .431,14.141 .250,14.807 .070 ; 0.9,0,9)$

((13.025.503, 13.784.588, 15.302.758, 16.061.843;1, 1),

(13.405.045, 14.164.131, 14.923.216, 15.682.301;0,9, 0,9$)$

((13.500.556, 14.360.367, 16.079.991, 16.939.803;1, 1),

(13.930.461, 14.790.273, 15.650.085, 16.509.897;0,9, 0,9$)$

((13.905.371, 14.873.968, 16.811.162, 17.779.759;1, 1),

(14.389.670, 15.358.266, 16.326.863, 17.295.460;0,9, 0,9)

((14.242.991, 15.329.075, 17.501.244, 18.587.329;1, 1),

(14.786.033, 15.872.117, 16.958.202, 18.044.287;0,9, 0,9)

((14.516.004, 15.728.976, 18.154.918, 19.367.890;1, 1),

(15.122.490, 16.335.461, 17.548.433, 18.761.404;0,9, 0,9)

((14.726.573, 16.076.582, 18.776.600, 20.126.609;1, 1),

(15.401.578, 16.751.587, 18.101.596, 19.451.605;0,9, 0,9)

((14.876.444, 16.374.454, 19.370.474, 20.868.483;1, 1),

$(15.625 .449,17.123 .459,18.621 .469,20.119 .479 ; 0,9,0,9)$

$((14.966 .966,16.624 .816,19.940 .517,21.598 .368 ; 1,1)$,
$(15.795 .891,17.453 .742,19.111 .592,20.769 .443 ; 0.9,0,9)$

((14.999.096, 16.829.575, 20.490.532, 22.321.011;1, 1),

(15.914.336, 17.744.814, 19.575.293, 21.405.771;0,9, 0,9))

$((14.973 .415,16.990 .332,21.024 .166,23.041 .083 ; 1,1)$,
$(15.981 .874,17.998 .791,20.015 .708,22.032 .624 ; 0,9,0,9))$

((14.890.129, 17.108.399, 21.544.939, 23.763.210;1, 1),

(15.999.264, 18.217.534, 20.435.804, 22.654.075;0,9, 0,9))

$((14.749 .072,17.184 .804,22.056 .268,24.491 .999 ; 1,1)$,
$(15.966 .938,18.402 .670,20.838 .402,23.274 .133 ; 0.9,0,9)$

$((8.045 .073,13.884 .536,25.563 .464,31.402 .927 ; 1,1)$,

$(10.964 .805,16.804 .268,22.643 .732,28.403 .927 ; 1,1)$
Book Value at End of Year

( $(20.080 .000,20.620 .000,21.700 .000,22.240 .000 ; 1,1)$,

(18.300.800, 18.884.000, 20.050.400, 20.633.600;1, 1),

$(18.592 .400,19.845 .000,20.050 .400,20.633 .600,1,1)$,

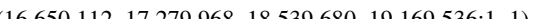

(16.965.040, 17.594.896, 18.224.752, 18.854.608:0,9,0,9)

(15.116.549, 15.796.794, 17.157.283, 17.837.527:1,1),

(15.456.671, 16.136.916, 16.817.160, 17.497.405;0.9,0,9))

(13.689.547, 14.424.211, 15.893.539, 16.628.203;1, 1),

$(14.056 .879,14.791 .543,15.526 .207,16.260 .871 ; 0,9,0,9))$

(12.359.291, 13.152.728, 14.739.602, 15.533.039;1, 1),

(15.342.884, 15.136.321:0.9, 0,9)

(11.116.648, 11.973.560, 13.687.384, 14.544.296;1, 1),

(12.

$(9.953 .104,10.878 .569,12.729 .499,13.654 .964 ; 1,1)$,

$415.836,11.341 .302,12.266 .767,13.192 .232 ; 0,9,0,9)$

$(8.860 .707,9.860 .209,11.859 .214,12.858 .716 ; 1,1)$,

$(9.360 .458,10.359 .960,11.359 .463,12.358 .965 ; 0,9,0,9)$

$(7.832 .009,8.911 .472,11.070 .397,12.149 .859 \cdot 1,1)$

(8.371.741, 9.451.203, 10.530.666, 11.610.128;0,9, 0,9))

( $(6.860 .021,8.025 .840,10.357 .479,11.523 .299 ; 1,1)$,

(5.938.157, 7.197.242 $9.715 .412,10.974 .497 .1,1)$

$(6.567 .699,7.826 .784,9.085 .869,10.344 .955 ; 0,9,0,9)$

$(5.060 .197,6.420 .009,9.139 .633,10.499 .444 \cdot 1,1)$

$(5.740 .103,7.099 .915,8.459 .727,9.819 .539 ; 0.9,0,9))$

$(4.220 .241,5.688 .838,8.626 .032,10.094 .629 ; 1,1)$

$(4.954 .540,6.423 .137,7.891 .734,9.360 .330 ; 0,9,0,9)$

( $(3.412 .671,4.998 .756,8.170 .925,9.757 .009 ; 1,1)$,

(2.632.110, 4.345.082, $7.771 .024,9.483 .996 ; 1,1)$

$(3.488 .596,5.201 .567,6.914 .539,8.627 .510 ; 0,9,0.9))$ $(1.873 .391,3.723 .400,7.423 .418,9.273 .427 ; 1,1)$

$(2.798 .395,4.648 .404,6.498 .413,8.348 .422 ; 0,9,0,9)$ $(1.131 .517,3.129 .526,7.125 .546,9.123 .556 ; 1,1)$

(1.632, 2.559.483, 6.875.184, 9.033.034:1, 1),

(

(

(

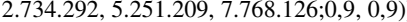

(-1.763.210, 955.061, 6.391.601, 9.109.871:1, 1),

(-2.491.999, 443.732.6.315.196, 9.250.928:1,1),

$(-1.024 .133,1.911 .598,4.847 .330,7.783 .062 ; 0,9,0,9)$

((2.340.000, 2.808.000, 3.744.000, 4.212.000;1. 1 ) 
Table 8: Details of breakdown for defuzzied Straight-Line depreciation method.

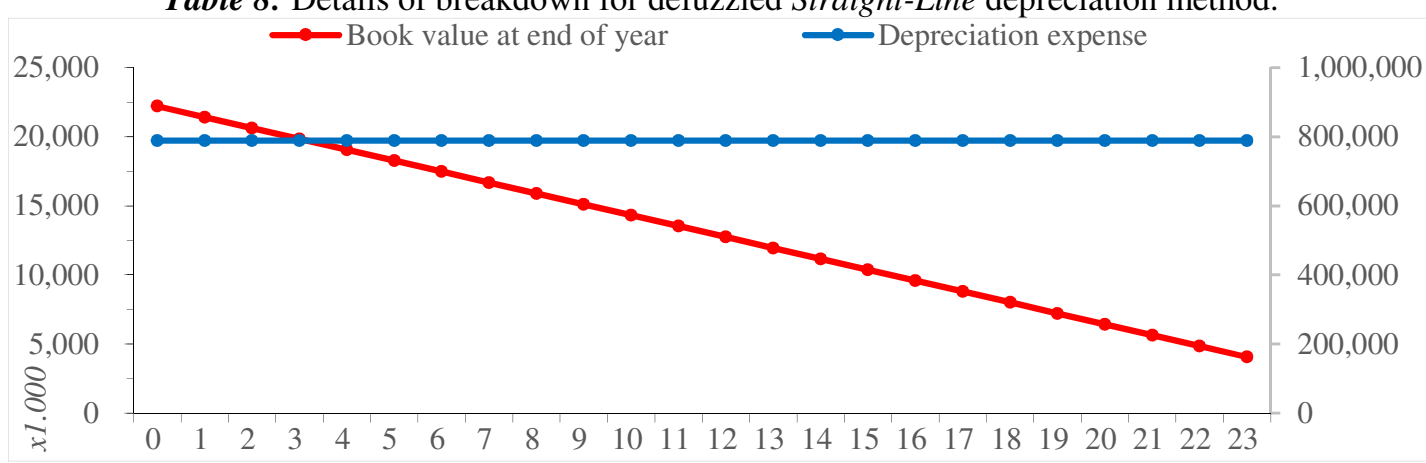

Table 9: Details of breakdown for defuzzied Double Declining Balance Depreciation method.

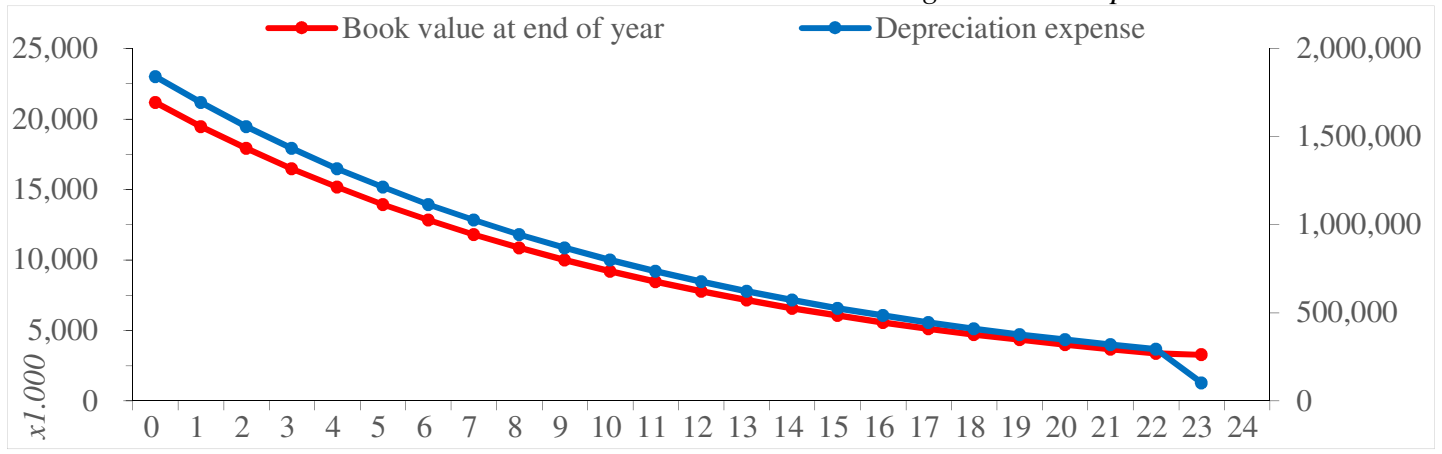

Table 1 is related to the price of assets, price of scrap metal, light ship displacement (LDT), salvage value, utilization life, useful life, age of the asset, annual depreciation, and annual deprecation defuzzfy. Also, it consists of the values of classic deprecation, trapezoidal interval type- 2 fuzzy deprecation, and triangular interval type-2 fuzzy deprecation for using interval type-2 straight-line depreciation and accelerated depreciation methods.

Table 2 is related to details of breakdown for classic straight-line depreciation method for 25 years. It consists of book value at beginning of the year, depreciation expense, accumulated depreciation, and book value at end of the year. Table 3: Details of breakdown for triangular interval type-2 fuzzy for StraightLine depreciation method

\begin{tabular}{|c|c|c|c|}
\hline Year & Book Value at Beginning of Year & Depreciation Expense & Accumulated Depreciation \\
\hline 1 & $\begin{array}{l}((22.000 .000,23.000 .000,24.000 .000 ; 1) \\
(22.250 .000,23.000 .000,23.750 .000 ; 0.9))\end{array}$ & $\begin{array}{l}((711.520,788.960,866.400 ; 1), \\
(730.880,788.960,847.040 ; 0.9))\end{array}$ & $\begin{array}{l}((711.520,788.960,866.400 ; 1) \\
(730.880,788.960,847.040 ; 0.9))\end{array}$ \\
\hline 2 & $\begin{array}{l}((21.133 .600,22.211 .040,23.288 .480 ; 1) \\
(21.402 .960,22.211 .040,23.019 .120 ; 0.9))\end{array}$ & $\begin{array}{l}((711.520,788.960,866.400 ; 1) \\
(730.880,788.960,847.040 ; 0.9))\end{array}$ & $\begin{array}{l}((1.423 .040,1.577 .920,1.732 .800 ; 1), \\
(1.461 .760,1.577 .920,1.694 .080 ; 0.9))\end{array}$ \\
\hline 3 & $\begin{array}{l}((20.267 .200,21.422 .080,22.576 .960 ; 1) \\
(20.555 .920,21.422 .080,22.288 .240 ; 0.9))\end{array}$ & $\begin{array}{l}((711.520,788.960,866.400 ; 1) \\
(730.880,788.960,847.040 ; 0.9))\end{array}$ & $\begin{array}{l}((2.134 .560,2.366 .880,2.599 .200 ; 1) \\
(2.192 .640,2.366 .880,2.541 .120 ; 0.9))\end{array}$ \\
\hline 4 & $\begin{array}{l}((19.400 .800,20.633 .120,21.865 .440 ; 1) \\
(19.708 .880,20.633 .120,21.557 .360 ; 0.9))\end{array}$ & $\begin{array}{l}((711.520,788.960,866.400 ; 1) \\
(730.880,788.960,847.040 ; 0.9))\end{array}$ & $\begin{array}{l}((2.846 .080,3.155 .840,3.465 .600 ; 1), \\
(2.923 .520,3.155 .840,3.388 .160 ; 0.9))\end{array}$ \\
\hline 5 & $\begin{array}{l}((18.534 .400,19.844 .160,21.153 .920 ; 1) \\
(18.861 .840,19.844 .160,20.826 .480 ; 0.9))\end{array}$ & $\begin{array}{l}((711.520,788.960,866.400 ; 1) \\
(730.880,788.960,847.040 ; 0.9))\end{array}$ & $\begin{array}{l}((3.557 .600,3.944 .800,4.332 .000 ; 1) \\
(3.654 .400,3.944 .800,4.235 .200 ; 0.9))\end{array}$ \\
\hline 6 & $\begin{array}{l}((17.668 .000,19.055 .200,20.442 .400 ; 1) \\
(18.014 .800,19.055 .200,20.095 .600 ; 0.9))\end{array}$ & $\begin{array}{l}((711.520,788.960,866.400 ; 1) \\
(730.880,788.960,847.040 ; 0.9))\end{array}$ & $\begin{array}{l}((4.269 .120,4.733 .760,5.198 .400 ; 1) \\
(4.385 .280,4.733 .760,5.082 .240 ; 0.9))\end{array}$ \\
\hline 7 & $\begin{array}{l}((16.801 .600,18.266 .240,19.730 .880 ; 1) \\
(17.167 .760,18.266 .240,19.364 .720 ; 0.9))\end{array}$ & $\begin{array}{l}((711.520,788.960,866.400 ; 1) \\
(730.880,788.960,847.040 ; 0.9))\end{array}$ & $\begin{array}{l}((4.980 .640,5.522 .720,6.064 .800 ; 1), \\
(5.116 .160,5.522 .720,5.929 .280 ; 0.9))\end{array}$ \\
\hline 8 & $\begin{array}{l}((15.935 .200,17.477 .280,19.019 .360 ; 1) \\
(16.320 .720,17.477 .280,18.633 .840 ; 0.9))\end{array}$ & $\begin{array}{l}((711.520,788.960,866.400 ; 1), \\
(730.880,788.960,847.040 ; 0.9))\end{array}$ & $\begin{array}{l}((5.692 .160,6.311 .680,6.931 .200 ; 1), \\
(5.847 .040,6.311 .680,6.776 .320 ; 0.9))\end{array}$ \\
\hline 9 & $\begin{array}{l}((15.068 .800,16.688 .320,18.307 .840 ; 1) \\
(15.473 .680,16.688 .320,17.902 .960 ; 0.9))\end{array}$ & $\begin{array}{l}((711.520,788.960,866.400 ; 1) \\
(730.880,788.960,847.040 ; 0.9))\end{array}$ & $\begin{array}{l}((6.403 .680,7.100 .640,7.797 .600 ; 1) \\
(6.577 .920,7.100 .640,7.623 .360 ; 0.9))\end{array}$ \\
\hline 10 & $\begin{array}{l}((14.202 .400,15.899 .360,17.596 .320 ; 1) \\
(14.626 .640,15.899 .360,17.172 .080 ; 0.9))\end{array}$ & $\begin{array}{l}((711.520,788.960,866.400 ; 1) \\
(730.880,788.960,847.040 ; 0.9))\end{array}$ & $\begin{array}{l}((7.115 .200,7.889 .600,8.664 .000 ; 1) \\
(7.308 .800,7.889 .600,8.470 .400 ; 0.9))\end{array}$ \\
\hline 11 & $\begin{array}{l}((13.336 .000,15.110 .400,16.884 .800 ; 1) \\
(13.779 .600,15.110 .400,16.441 .200 ; 0.9))\end{array}$ & $\begin{array}{l}((711.520,788.960,866.400 ; 1) \\
(730.880,788.960,847.040 ; 0.9))\end{array}$ & $\begin{array}{l}((7.826 .720,8.678 .560,9.530 .400 ; 1) \\
(8.039 .680,8.678 .560,9.317 .440 ; 0.9))\end{array}$ \\
\hline 12 & $\begin{array}{l}((12.469 .600,14.321 .440,16.173 .280 ; 1) \\
(12.932 .560,14.321 .440,15.710 .320 ; 0.9))\end{array}$ & $\begin{array}{l}((711.520,788.960,866.400 ; 1) \\
(730.880,788.960,847.040 ; 0.9))\end{array}$ & $\begin{array}{l}((8.538 .240,9.467 .520,10.396 .800 ; 1) \\
(8.770 .560,9.467 .520,10.164 .480 ; 0.9))\end{array}$ \\
\hline 13 & $\begin{array}{l}((11.603 .200,13.532 .480,15.461 .760 ; 1) \\
(12.085 .520,13.532 .480,14.979 .440 ; 0.9))\end{array}$ & $\begin{array}{l}((711.520,788.960,866.400 ; 1) \\
(730.880,788.960,847.040 ; 0.9))\end{array}$ & $\begin{array}{l}((9.249 .760,10.256 .480,11.263 .200 ; 1) \\
(9.501 .440,10.256 .480,11.011 .520 ; 0.9))\end{array}$ \\
\hline 14 & $\begin{array}{l}((10.736 .800,12.743 .520,14.750 .240 ; 1) \\
(11.238 .480,12.743 .520,14.248 .560 ; 0.9))\end{array}$ & $\begin{array}{l}((711.520,788.960,866.400 ; 1) \\
(730.880,788.960,847.040 ; 0.9))\end{array}$ & $\begin{array}{l}((9.961 .280,11.045 .440,12.129 .600 ; 1) \\
(10.232 .320,11.045 .440,11.858 .560 ; 0.9))\end{array}$ \\
\hline
\end{tabular}


$17 \quad((8.137 .600,10.376 .640,12.615 .680 ; 1)$ $(8.697 .360,10.376 .640,12.055 .920 ; 0.9))$ ((7.271.200, 9.587.680, 11.904.160;1),

$18 \quad(7.850 .320,9.587 .680,11.325 .040 ; 0.9))$

$19 \quad((6.404 .800,8.798 .720,11.192 .640 ; 1)$ (7.003.280, 8.798.720, 10.594.160;0.9)) $((5.538 .400,8.009 .760,10.481 .120 ; 1)$,

$20 \quad(6.156 .240,8.009 .760,9.863 .280 ; 0.9))$

$21 \quad((4.672 .000,7.220 .800,9.769 .600 ; 1)$ $(5.309 .200,7.220 .800,9.132 .400 ; 0.9))$ $((3.805 .600,6.431 .840,9.058 .080 ; 1)$,

$22 \quad(4.462 .160,6.431 .840,8.401 .520 ; 0.9))$ ((2.939.200, 5.642.880, 8.346.560;1),

$23(3.615 .120,5.642 .880,7.670 .640 ; 0.9))$ $((2.072 .800,4.853 .920,7.635 .040 ; 1)$,

$24 \quad(2.768 .080,4.853 .920,6.939 .760 ; 0.9))$ ((1.206.400, 4.064.960, 6.923.520;1)

$25 \quad(1.206 .400,4.064 .960,6.923 .520 ; 1)$,
$((711.520,788.960,866.400 ; 1)$ $(730.880,788.960,847.040 ; 0.9))$ $((711.520,788.960,866.400 ; 1)$, $(730.880,788.960,847.040 ; 0.9))$ ((711.520, 788.960, 866.400;1), $(730.880,788.960,847.040 ; 0.9))$ $((711.520,788.960,866.400 ; 1)$, $(730.880,788.960,847.040 ; 0.9))$ ((711.520, 788.960, 866.400;1), $(730.880,788.960,847.040 ; 0.9))$ ((711.520, 788.960, 866.400;1), $(730.880,788.960,847.040 ; 0.9))$ $((711.520,788.960,866.400 ; 1)$, $(730.880,788.960,847.040 ; 0.9))$ ((711.520, 788.960, 866.400;1), $(730.880,788.960,847.040 ; 0.9))$ ((711.520, 788.960, 866.400;1), $(730.880,788.960,847.040 ; 0.9))$ $((711.520,788.960,866.400 ; 1)$ $(730.880,788.960,847.040 ; 0.9))$ ((711.520, 788.960, 866.400;1), $(730.880,788.960,847.040 ; 0.9))$
((10.672.800, 11.834.400, 12.996.000;1), $(10.963 .200,11.834 .400,12.705 .600 ; 0.9))$ ((11.384.320, 12.623.360, 13.862.400;1), $(11.694 .080,12.623 .360,13.552 .640 ; 0.9))$ ((12.095.840, 13.412.320, 14.728.800;1), $(12.424 .960,13.412 .320,14.399 .680 ; 0.9))$ ((12.807.360, 14.201.280, 15.595.200;1), $(13.155 .840,14.201 .280,15.246 .720 ; 0.9))$ ((13.518.880, 14.990.240, 16.461.600;1), $(13.886 .720,14.990 .240,16.093 .760 ; 0.9))$ $((14.230 .400,15.779 .200,17.328 .000 ; 1)$ $(14.617 .600,15.779 .200,16.940 .800 ; 0.9))$ ((14.941.920, 16.568.160, 18.194.400;1), $(15.348 .480,16.568 .160,17.787 .840 ; 0.9))$ $((15.653 .440,17.357 .120,19.060 .800 ; 1)$, $(16.079 .360,17.357 .120,18.634 .880 ; 0.9))$ ((16.364.960, 18.146.080, 19.927.200;1), $(16.810 .240,18.146 .080,19.481 .920 ; 0.9))$ $((17.076 .480,18.935 .040,20.793 .600 ; 1)$ $(17.541 .120,18.935 .040,20.328 .960 ; 0.9))$ ((17.788.000, 19.724.000, 21.660.000;1), $(18.272 .000,19.724 .000,21.176 .000 ; 0.9))$

Table 4 is related to details of breakdown for trapezoidal interval type-2 fuzzy for straight-line depreciation method for 25 years. It consists of book value at beginning of the year, depreciation expense, accumulated depreciation, and book value at end of the year. Error! Reference source not found. is related to details of breakdown for triangular interval type-2 fuzzy for straight-line depreciation method for 25 years. It consists of book value at beginning of the year, depreciation expense, accumulated depreciation, and book value at end of the year. Table 8 is related to details of the breakdown for the defuzzied straight-line depreciation method.

Table 5 is related to details of breakdown for classic double-declining balance depreciation method for 25 years. It consists of book value at beginning of the year, depreciation expense, accumulated depreciation, and book value at end of the year. Table 6: Details of breakdown for triangular interval type-2 fuzzy for Double Declining Balance Depreciation method.

\begin{tabular}{|c|c|c|c|}
\hline Year & Book Value at Beginning of Year & Depreciation Expense & Accumulated Depreciation \\
\hline 1 & $\begin{array}{l}((22.000 .000,23.000 .000,24.000 .000 ; 1) \\
(22.250 .000,23.000 .000,23.750 .000 ; 0,9))\end{array}$ & $\begin{array}{l}((1.760 .000,1.840 .000,1.920 .000 ; 1), \\
(1.780 .000,1.840 .000,1.900 .000 ; 0,9))\end{array}$ & $\begin{array}{l}((1.760 .000,1.840 .000,1.920 .000 ; 1), \\
(1.780 .000,1.840 .000,1.900 .000 ; 0,9))\end{array}$ \\
\hline 2 & $\begin{array}{l}((20.080 .000,21.160 .000,22.240 .000 ; 1) \\
(20.350 .000,21.160 .000,21.970 .000 ; 0,9))\end{array}$ & $\begin{array}{l}((1.606 .400,1.692 .800,1.779 .200 ; 1), \\
(1.628 .000,1.692 .800,1.757 .600 ; 0,9))\end{array}$ & $\begin{array}{l}((3.366 .400,3.532 .800,3.699 .200 ; 1) \\
(3.408 .000,3.532 .800,3.657 .600 ; 0,9))\end{array}$ \\
\hline 3 & $\begin{array}{l}((18.300 .800,19.467 .200,20.633 .600 ; 1) \\
(18.592 .400,19.467 .200,20.342 .000 ; 0,9))\end{array}$ & $\begin{array}{l}((1.464 .064,1.557 .376,1.650 .688 ; 1), \\
(1.487 .392,1.557 .376,1.627 .360 ; 0,9))\end{array}$ & $\begin{array}{l}((4.830 .464,5.090 .176,5.349 .888 ; 1), \\
(4.895 .392,5.090 .176,5.284 .960 ; 0,9))\end{array}$ \\
\hline 4 & $\begin{array}{l}((16.650 .112,17.909 .824,19.169 .536 ; 1) \\
(16.965 .040,17.909 .824,18.854 .608 ; 0,9))\end{array}$ & $\begin{array}{l}((1.332 .009,1.432 .786,1.533 .563 ; 1) \\
(1.357 .203,1.432 .786,1.508 .369 ; 0,9))\end{array}$ & $\begin{array}{l}((6.162 .473,6.522 .962,6.883 .451 ; 1) \\
(6.252 .595,6.522 .962,6.793 .329 ; 0,9))\end{array}$ \\
\hline 5 & $\begin{array}{l}((15.116 .549,16.477 .038,17.837 .527 ; 1) \\
(15.456 .671,16.477 .038,17.497 .405 ; 0,9))\end{array}$ & $\begin{array}{l}((1.209 .324,1.318 .163,1.427 .002 ; 1) \\
(1.236 .534,1.318 .163,1.399 .792 ; 0,9))\end{array}$ & $\begin{array}{l}((7.371 .797,7.841 .125,8.310 .453 ; 1), \\
(7.489 .129,7.841 .125,8.193 .121 ; 0,9))\end{array}$ \\
\hline 6 & $\begin{array}{l}((13.689 .547,15.158 .875,16.628 .203 ; 1) \\
(14.056 .879,15.158 .875,16.260 .871 ; 0,9))\end{array}$ & $\begin{array}{l}((1.095 .164,1.212 .710,1.330 .256 ; 1) \\
(1.124 .550,1.212 .710,1.300 .870 ; 0,9))\end{array}$ & $\begin{array}{l}((8.466 .961,9.053 .835,9.640 .709 ; 1) \\
(8.613 .679,9.053 .835,9.493 .991 ; 0,9))\end{array}$ \\
\hline 7 & $\begin{array}{l}((12.359 .291,13.946 .165,15.533 .039 ; 1) \\
(12.756 .009,13.946 .165,15.136 .321 ; 0,9))\end{array}$ & $\begin{array}{l}((988.743,1.115 .693,1.242 .643 ; 1) \\
(1.020 .481,1.115 .693,1.210 .906 ; 0,9))\end{array}$ & $\begin{array}{l}((9.455 .704,10.169 .528,10.883 .352 ; 1) \\
(9.634 .160,10.169 .528,10.704 .896 ; 0,9))\end{array}$ \\
\hline 8 & $\begin{array}{l}((11.116 .648,12.830 .472,14.544 .296 ; 1) \\
(11.545 .104,12.830 .472,14.115 .840 ; 0,9))\end{array}$ & $\begin{array}{l}((889.332,1.026 .438,1.163 .544 ; 1) \\
(923.608,1.026 .438,1.129 .267 ; 0,9))\end{array}$ & $\begin{array}{l}((10.345 .036,11.195 .966,12.046 .896 ; 1) \\
(10.557 .768,11.195 .966,11.834 .164 ; 0,9))\end{array}$ \\
\hline 9 & $\begin{array}{l}((9.953 .104,11.804 .034,13.654 .964 ; 1) \\
(10.415 .836,11.804 .034,13.192 .232 ; 0,9))\end{array}$ & $\begin{array}{l}((796.248,944.323,1.092 .397 ; 1) \\
(833.267,944.323,1.055 .379 ; 0,9))\end{array}$ & $\begin{array}{l}((11.141 .284,12.140 .289,13.139 .293 ; 1), \\
(11.391 .035,12.140 .289,12.889 .542 ; 0,9))\end{array}$ \\
\hline 10 & $\begin{array}{l}((8.860 .707,10.859 .711,12.858 .716 ; 1) \\
(9.360 .458,10.859 .711,12.358 .965 ; 0,9))\end{array}$ & $\begin{array}{l}((708.857,868.777,1.028 .697 ; 1) \\
(748.837,868.777,988.717 ; 0,9))\end{array}$ & $\begin{array}{l}((11.850 .141,13.009 .066,14.167 .991 ; 1), \\
(12.139 .872,13.009 .066,13.878 .259 ; 0,9))\end{array}$ \\
\hline 11 & $\begin{array}{l}((7.832 .009,9.990 .934,12.149 .859 ; 1) \\
(8.371 .741,9.990 .934,11.610 .128 ; 0,9))\end{array}$ & $\begin{array}{l}((626.561,799.275,971.989 ; 1) \\
(669.739,799.275,928.810 ; 0,9))\end{array}$ & $\begin{array}{l}((12.476 .701,13.808 .340,15.139 .979 ; 1), \\
(12.809 .611,13.808 .340,14.807 .070 ; 0,9))\end{array}$ \\
\hline 12 & $\begin{array}{l}((6.860 .021,9.191 .660,11.523 .299 ; 1) \\
(7.442 .930,9.191 .660,10.940 .389 ; 0,9))\end{array}$ & $\begin{array}{l}((548.802,735.333,921.864 ; 1) \\
(595.434,735.333,875.231 ; 0,9))\end{array}$ & $\begin{array}{l}((13.025 .503,14.543 .673,16.061 .843 ; 1), \\
(13.405 .045,14.543 .673,15.682 .301 ; 0,9))\end{array}$ \\
\hline 13 & $\begin{array}{l}((5.938 .157,8.456 .327,10.974 .497 ; 1), \\
(6.567 .699,8.456 .327,10.344 .955 ; 0,9))\end{array}$ & $\begin{array}{l}((475.053,676.506,877.960 ; 1) \\
(525.416,676.506,827.596 ; 0,9))\end{array}$ & $\begin{array}{l}((13.500 .556,15.220 .179,16.939 .803 ; 1), \\
(13.930 .461,15.220 .179,16.509 .897 ; 0,9))\end{array}$ \\
\hline 14 & $\begin{array}{l}((5.060 .197,7.779 .821,10.499 .444 ; 1) \\
(5.740 .103,7.779 .821,9.819 .539 ; 0,9))\end{array}$ & $\begin{array}{l}((404.816,622.386,839.956 ; 1) \\
(459.208,622.386,785.563 ; 0,9))\end{array}$ & $\begin{array}{l}((13.905 .371,15.842 .565,17.779 .759 ; 1), \\
(14.389 .670,15.842 .565,17.295 .460 ; 0,9))\end{array}$ \\
\hline 15 & $\begin{array}{l}((4.220 .241,7.157 .435,10.094 .629 ; 1) \\
(4.954 .540,7.157 .435,9.360 .330 ; 0,9))\end{array}$ & $\begin{array}{l}((337.619,572.595,807.570 ; 1) \\
(396.363,572.595,748.826 ; 0,9))\end{array}$ & $\begin{array}{l}((14.242 .991,16.415 .160,18.587 .329 ; 1), \\
(14.786 .033,16.415 .160,18.044 .287 ; 0,9))\end{array}$ \\
\hline 16 & $\begin{array}{l}((3.412 .671,6.584 .840,9.757 .009 ; 1), \\
(4.205 .713,6.584 .840,8.963 .967 ; 0,9))\end{array}$ & $\begin{array}{l}((273.014,526.787,780.561 ; 1) \\
(336.457,526.787,717.117 ; 0,9))\end{array}$ & $\begin{array}{l}((14.516 .004,16.941 .947,19.367 .890 ; 1), \\
(15.122 .490,16.941 .947,18.761 .404 ; 0,9))\end{array}$ \\
\hline 17 & $\begin{array}{l}((2.632 .110,6.058 .053,9.483 .996 ; 1), \\
(3.488 .596,6.058 .053,8.627 .510 ; 0,9))\end{array}$ & $\begin{array}{l}((210.569,484.644,758.720 ; 1) \\
(279.088,484.644,690.201 ; 0,9))\end{array}$ & $\begin{array}{l}((14.726 .573,17.426 .591,20.126 .609 ; 1), \\
(15.401 .578,17.426 .591,19.451 .605 ; 0,9))\end{array}$ \\
\hline 18 & $\begin{array}{l}((1.873 .391,5.573 .409,9.273 .427 ; 1), \\
(2.798 .395,5.573 .409,8.348 .422 ; 0,9))\end{array}$ & $\begin{array}{l}((149.871,445.873,741.874 ; 1) \\
(223.872,445.873,667.874 ; 0,9))\end{array}$ & $\begin{array}{l}((14.876 .444,17.872 .464,20.868 .483 ; 1), \\
(15.625 .449,17.872 .464,20.119 .479 ; 0,9))\end{array}$ \\
\hline 19 & $\begin{array}{l}((1.131 .517,5.127 .536,9.123 .556 ; 1) \\
(2.130 .521,5.127 .536,8.124 .551 ; 0,9))\end{array}$ & $\begin{array}{l}((90.521,410.203,729.884 ; 1) \\
(170.442,410.203,649.964 ; 0,9))\end{array}$ & $\begin{array}{l}((14.966 .966,18.282 .667,21.598 .368 ; 1) \\
(15.795 .891,18.282 .667,20.769 .443 ; 0,9))\end{array}$ \\
\hline
\end{tabular}


((401.632, 4.717.333, 9.033.034;1)

$(1.480 .557,4.717 .333,7.954 .109 ; 0,9))$

$((-321.011,4.339 .947,9.000 .904 ; 1)$,

$(844.229,4.339 .947,7.835 .664 ; 0,9))$

$((-1.041 .083,3.992 .751,9.026 .585 ; 1)$,

$(217.376,3.992 .751,7.768 .126 ; 0,9))$

$((-1.763 .210,3.673 .331,9.109 .871 ; 1)$,

$(-404.075,3.673 .331,7.750 .736 ; 0,9))$

((-2.491.999, 3.379.464, 9.250.928; 1),

(-1.024.133, 3.379.464, 7.783.062; 0.9))
((32.131, 377.387, 722.643;1),

$(118.445,377.387,636.329 ; 0,9))$

$((-25.681,347.196,720.072 ; 1)$,

$(67.538,347.196,626.853 ; 0,9))$

$((-83.287,319.420,722.127 ; 1)$,

$(17.390,319.420,621.450 ; 0,9))$

$((-141.057,293.866,728.790 ; 1)$,

$(-32.326,293.866,620.059 ; 0,9))$

$((-6.703 .999,103.464,6.910 .928 ; 1)$,

$(-5.002 .133,103.464,5.209 .062 ; 0.9))$
((14.999.096, 18.660.053, 22.321.011;1), $(15.914 .336,18.660 .053,21.405 .771 ; 0.9))$ ((14.973.415, 19.007.249, 23.041.083;1), $(15.981 .874,19.007 .249,22.032 .624 ; 0,9))$ ((14.890.129, 19.326.669, 23.763.210;1), $(15.999 .264,19.326 .669,22.654 .075 ; 0,9))$ ((14.749.072, 19.620.536, 24.491.999;1), $(15.966 .938,19.620 .536,23.274 .133 ; 0,9))$ ((8.045.073, 19.724.000, 31.402.927;1), $(10.964 .805,19.724 .000,28.483 .195 ; 0.9))$

25

Table 7 is related to details of breakdown for trapezoidal interval type-2 fuzzy for double-declining balance depreciation method for 25 years. It consists of book value at beginning of the year, depreciation expense, accumulated depreciation, and book value at end of the year. Error! Reference source not found. is related to details of breakdown for triangular interval type-2 fuzzy for double-declining balance depreciation method for 25 years. It consists of details of breakdown for triangular interval type-2 fuzzy for accelerated depreciation method. Table 9 is related to details of breakdown for defuzzied accelerated depreciation method. Because of the characteristic of the double-declining balance depreciation method, the allocation for 24 years.

\section{DISCUSSION AND CONCLUSION}

The shipping industry has a naturally uncertain environment as its ecosystem depends on many factors, as such the complication of the shipping investment environment, the volatility of the shipping market, the enormous amount of investment required, and the long payback period. Hence, to properly carry out ship investment evaluation under uncertain environmental conditions, these factors must be considered by estimating them adequately. However, the uncertainties in shipping affect the evaluation required to make a decision. For instance, the Baltic Dry Index has changed by around 200\% since last year (Trading Economics, 2021). The dry bulk shipping industry is having a highly volatile circular industry that the size and return of investment capital, and freight revenues are highly volatile (Greenwood and Hanson, 2015). The demand for service of the shipping industry is volatile and is directed by the seaborne trading which, in turn, is connected to the level of global economic activity, nevertheless, there is only a weak correlation between growth in global seaborne trade and global economic growth (Stopford, 2009). This is because the trading of bulk commodities such as principally grains, coal, and iron ore is affected by evolving geographic trade models (the location of commodity users as to commodity suppliers) and geopolitical events (e.g., the 1967-1975 Suez Canal closure, the 1979 Iranian revolution, the 1990 Gulf War, and the 2003 Iraq War, 2008 economic crisis, 2019 Covid-19 pandemic). Demanding of service in shipping is generally thought to be quite inelastic because there are few cost-effective alternatives for international transport of most bulk goods (Stopford, 2009; Greenwood and Hanson, 2015). Therefore, it has great uncertainty due to the nature of the shipping industry. When consideration the shipping industry ecosystem, affects the financial cash flow of the ship directly or indirectly due to the uncertainty it contains.

Merchant ships had a limited lifespan, Stopford (2009) determined the economic life of these ships as 25 years according to the results of the studies. Estimating the future cash flow in the ship investment that requires high investment is very difficult due to the dynamics of the sector. Shipowner as an investor aims to estimate the cash flow values as accurately as possible while making the investment decision. In such environments where uncertainty is high, fuzzy sets provide a wider solution than classical methods. Therefore, fuzzy sets have been used to provide feasible solutions for evaluating investment decision-making problems that take into consideration the uncertainty of the environment. Furthermore, interval type-2 fuzzy gives a more specific solution cluster and decision-makers are not forced to define a single membership function and have greater flexibility to define the membership functions of investment parameters. Thanks to the membership functions of interval type- 2 fuzzy sets, all the independent variables that constitute the cash flow of ship investment can be defined as interval type- 2 sets. Because of the investor, the future always contains uncertainty, however, the uncertainty of the future is limited by the fuzzy sets as they give a wide cluster for solutions.

There are many different depreciation methods in the literature however, Stopford (2009) mentioned the straight-line depreciation method as one of the most used methods in calculating depreciation in the 
shipping industry. Hence, in this study, straight-line depreciation and double-declining balance depreciation methods are proposed in terms of interval type- 2 fuzzy sets. The proposed IT2FS depreciation and classic depreciation methods were used to calculate a new ship's depreciation for 25 years in the study. The principal variables for calculating a ship's depreciation are the Price of Assets, Salvage Value, and Useful Life. However, when calculating the depreciation of a ship, the Salvage Value varies depending on Light Ship Displacement (LDT) and the Price of Scrap Metal. In this study, Price of Assets and Salvage Value variables are defined as IT2FSs. However, in defining the Salvage Value, the only Price of Scrap Metal is defined as IT2FSs. Because ship's depreciation calculation, Light Ship Displacement (LTD) and Useful Life values were fixed and undefined as IT2FSs, these values are defined as determinists.

Uncertainty is related to the future in this study. Therefore, in terms of the ship's investment evaluation, the depreciation will be important in determining the investment strategy of the organization, in its approach to the tax shield, and even in determining the second-hand ship sales strategy. Furthermore; in shipping investment, there are several methods of financing models, such as cash, leasing, $K G$ (Kommanditgesellschaft) system equity, KS (Kommandittselskap) private equity, private equity, public equity, corporate bonds, the ship found, mortgage-backed loans, bank loans, bank financing, unsecured/corporate loans, new building financing, mezzanine, special purpose acquisition companies, high pay-out structures, and master limited partnerships (Stopford, 2009; Schinas et al., 2015; Kavussanos and Visvikis, 2016). Therefore, the depreciation will be taken into account when choosing the possible investment model depending on the financing model that the organization will choose, the type of ship to be preferred, and the capacity of the ship. Because, in terms of considering the tax shield, an organization that invests using outsourcing may prefer a strategy to show high depreciation, especially in the first years of the investment. Hence, the double-declining balance depreciation method can be preferred. In addition, there may be expectations that may arise in the following years depending on the cash flow. The organization may prefer the strategy of paying less tax by showing the depreciation expense high in the first years of the investment. This can protect from possible financial cash flow uncertainty in the first years of the business. When viewed from another aspect, it will be important for public shipping companies, especially those with new investments, to show high profitability. This situation can be evaluated from two aspects: (1) The company to be opened to the new public, may prefer a model in which the business has higher profitability in the first years of the investment with the depreciation model to be used in the investment evaluation. Hence, it will highlight the profitability of the companies before being the public shipping companies. (2) The public shipping companies may have the choice of strategy to show higher profits in the first years of the new investment. In this case, the value of the company's stock prices will ensure that the investment is high in the first years. Both situations will change depending on the purpose and strategy of the company.

In addition, a method of determining the value of the second-hand ship is to determine the second-hand price of the ship by deducting the value of the new ship as much as the depreciation amount for the relevant age of the ship (Beenstock, 1985). Along with this relationship, calculating the depreciation value with a more accurate approach, taking into account the uncertainties, will provide convenience for decision-makers. However, there will be differences in depreciation amounts depending on alternative ship types and sizes before the investment. Because the second-hand price evaluation of the ship type with high-value depreciation will also differ. The depreciation also can be taken place as a factor in the second-hand values because of the occurrence of these differences. Therefore, it will also be a criterion when this situation is evaluated in terms of decision-makers.

In all cases, when the calculation of depreciation in terms of especially an investment perspective along with the natural uncertainties in the shipping industry, this uncertainty with the proposed method apart from classical deterministic depreciation methods so, it will further increase the accuracy in the depreciation calculations by taking into consideration the uncertainty. This will contribute to making more predictable decisions for decision-makers in the organization. Because deterministic models are insufficient in the face of this uncertainty. For instance, when we look into the future to calculate the scrap value of a ship, one of the best approaches to determine the future values of the depreciation 
components, which are the Price of Scrap Metal and Value of Assets, will be with fuzzy logic, so the depreciation considering as an investment perspective is a factor that affects the ship's cash flow.

This study along with the recommendation of straight-line depreciation and accelerated depreciation methods in interval type- 2 methods, will contribute especially to the points where classical methods are insufficient. Before the investment decisions, when determining the investment strategies, choosing the depreciation calculation type, and determining the tax strategy, the interval type-2 depreciation methods will provide more accurate input knowledge to the decision-makers because of the uncertainty of the fuzzy sets.

In this study, interval type-2 triangular and trapezoidal straight-line depreciation and accelerated depreciation methods are proposed to the literature, and a ship's depreciation calculations are carried out and compared to classical depreciation methods. The results presented that it can be successfully applied in terms of using interval type-2 fuzzy sets in every aspect.

Author Contributions Both authors contributed to introduction, literature, conceptualization, methodology, writing and conclusion.

\section{Declaration}

Conflicts of interest The authors declare that they have no conflict of interest.

Ethical approval This material is the authors' own original work, which has not been previously published elsewhere. The paper is not currently being considered for publication elsewhere. The paper reflects the authors' own research and analysis in a truthful and complete manner.

Funding details (In case of Funding) No funds provided.

Informed Consent Not applicable. 


\section{REFERENCES}

Abdel-Khalik, A. R. (1975). Advertising Effectiveness and Accounting Policy. The Accounting Review, 50(4), 657-670.

Albonico, A., Kalyvitis, S., \& Pappa, E. (2014). Capital maintenance and depreciation over the business cycle. Journal of Economic Dynamics and Control, 39, 273-286. https://doi.org/10.1016/j.jedc.2013.12.008

Ayers, B. C., Lefanowicz, C. E., \& Robinson, J. R. (2000). The Effects of Goodwill Tax Deductions on the Market for Corporate Acquisitions. Journal of the American Taxation Association, 22(s-1), 34-50. https://doi.org/10.2308/jata.2000.22.s-1.34

Baumol, W. J. (1971). Optimal Depreciation Policy: Pricing the Products of Durable Assets. The Bell Journal of Economics and Management Science, 2(2), 638-656.

Beenstock, M. (1985). A theory of ship prices. Maritime Policy and Management, 12(3), 215-225.

Bellman. R.E. \& Zadeh. L.A. (1970). Decision-making in a fuzzy environment. Management Science. vol.17. no.4. pp.141-164.

Berg, M., \& Moore, G. (1989). The Choice of Depreciation Method Under Uncertainty. Decision Sciences, 20(4), 643-654. https://doi.org/10.1111/j.1540-5915.1989.tb01409.x

Berg, M., Waegenaere, A. De, \& Wielhouwer, J. L. (2001). Theory and Methodology: Optimal tax depreciation with uncertain future cash-flows. European Journal of Operational Research, 132(1), 197-209. https://doi.org/10.1016/S0377-2217(00)00132-6

Bokhari, S., \& Geltner, D. (2018). Characteristics of Depreciation in Commercial and Multifamily Property: An Investment Perspective. Real Estate Economics, 46(4), 745-782. https://doi.org/10.1111/1540-6229.12156

Buckley. J.J. (1985). Fuzzy hierarchical analysis. Fuzzy Set. Syst.. 17. 233-247.

Çankaya, F., \& Yilmaz, Z. (2014). Üretim Miktarına Göre Amortisman Yönteminin Değişken Maliyetler ve Kârlılık Üzerine Etkileri. Bil. Derg, 8, 221-242.

Davidson, S., \& Drake, D. F. (1961). Capital Budgeting and the "Best" Tax Depreciation Method. The Journal of Business, 34(4).

de Rassenfosse, G., \& Jaffe, A. B. (2018). Econometric evidence on the depreciation of innovations. European Economic Review, 101, 625-642. https://doi.org/10.1016/j.euroecorev.2017.11.005

De Waegenaere, A., \& Wielhouwer, J. L. (2002). Optimal tax depreciation lives and charges under regulatory constraints. OR Spectrum, 24(2), 151-177. https://doi.org/10.1007/s00291-002-0096-0

Duvall, L., Jennings, R., Robinson, J., \& Thompson, R. (1992). Can Investors Unravel the Effects of Goodwill Accounting? Accounting Horizons, 6-2.

Engin, D., \& Atabay, E. (2018). Depreciation Procedures by Accountings Systems in Turkey and Evaluation Regarding Tax Effect. Journal of Accounting, Finance and Auditing Studies, 4(2), 67-91.

Falk, H., \& Miller, J. C. (1977). Amortization of Advertising Expenditures. Journal of Accounting Research, 15(1), $12-22$.

Femminis, G. (2008). Risk-aversion and the investment-uncertainty relationship: The role of capital depreciation. Journal of Economic Behavior and Organization, 65(3-4), 585-591. https://doi.org/10.1016/j.jebo.2006.03.003

Gabriel, A. D. P. (1937). Valuation and Amortization. The Accounting Review, 12(3), 209-226.

Glaum, M., Landsman, W. R., \& Wyrwa, S. (2015). Determinants of Goodwill Impairment: International Evidence. SSRN Electronic Journal. https://doi.org/10.2139/ssrn.2608425

Greenwood, R., \& Hanson, S. G. (2015). Waves in ship prices and investment. The Quarterly Journal of Economics, 130(1), 55-109.

Hall, S. C. (1993). Determinants of goodwill amortization period. Journal of Business Finance \& Accounting, 20(4), 613-621. https://doi.org/10.1111/j.1468-5957.1993.tb00279.x

Henning, S. L., \& Shaw, W. H. (2003). Is the selection of the amortization period for goodwill a strategic choice? Review of Quantitative Finance and Accounting, 20(4), 315-333. https://doi.org/10.1023/A:1024043316292

Hirschey, M., \& Weygandt, J. J. (1985). Amortization Policy for Advertising and Research and Development Expenditures. Journal of Accounting Research, 23(1), 326-335.

Hsieh, T. Y., Lu, S. T., \& Tzeng, G. H. (2004). Fuzzy MCDM approach for planning and design tender's selection in public office buildings. International Journal of Project Management, 22(7), 573-584

Huefner, R. J., \& Largay, J. A. (2004). The Effect of the New Goodwill Accounting Rules on Financial Statements. The CPA Journal, 74(10), 30-35.

International Accounting Standards (IAS), (2021), IAS 16 - Property, Plant and Equipment, https://www.iasplus.com/en-gb/standards/ias/ias16

Jackson, S. B. (2008). The effect of firms' depreciation method choice on managers' capital investment decisions. Accounting Review, 83(2), 351-376. https://doi.org/10.2308/accr.2008.83.2.351 
Jackson, S. B., (Kelvin) Liu, X., \& Cecchini, M. (2009). Economic consequences of firms' depreciation method choice: Evidence from capital investments. Journal of Accounting and Economics, 48(1), 54-68. https://doi.org/10.1016/j.jacceco.2009.06.001

Jackson, S. B., Rodgers, T. C., \& Tuttle, B. (2010). The effect of depreciation method choice on asset selling prices. Accounting, Organizations and Society, 35(8), 757-774. https://doi.org/10.1016/j.aos.2010.09.004

Jennergren, L. P. (2018). A note on the linear and annuity class of depreciation methods. International Journal of Production Economics, 204(April), 123-134. https://doi.org/10.1016/j.ijpe.2018.05.004

Jennings, R., LeClere, M., \& Thompson, R. B. (2001). Goodwill Amortization and the Usefulness of Earnings. Financial Analysts Journal, 57(5), 20-28. https://doi.org/10.2469/faj.v57.n5.2478

Kavussanos, M.G. \& Visvikis, I.D. (2016). Shipping Markets and Their Economic Drivers. In the International Handbook of Shipping Finance: theory and practice. Springer. https://doi.org/10.1057/978-1-137-46546$7 \_1$

Khalili, S., Mehrjerdi, Y. Z., \& Zare, H. K. (2014). Choosing the best method of depreciating assets and after-tax economic analysis under uncertainty using fuzzy approach. Decision Science Letters, 3(4), 457-466. https://doi.org/10.5267/j.ds1.2014.8.001

Kulp, A., \& Hartman, J. C. (2011). Optimal tax depreciation with loss carry-forward and backward options. European Journal of Operational Research, 208(2), 161-169. https://doi.org/10.1016/j.ejor.2010.06.040

Lev, B., \& Sougiannis, T. (1996). The capitalization, amortization, and value-relevance of R\&D. Journal of Accounting and Economics, 21(1), 107-138. https://doi.org/10.1016/0165-4101(95)00410-6

Mendel. J.M. (2007). Advances in type-2 fuzzy sets and systems. Inform. Sci.. 177(1) 84-110.

Mendel. J.M., John. R.I. \& Liu. F. (2006). Interval type-2 fuzzy logic systems made simple. IEEE Trans. Fuzzy Syst.. 14 (6). 808-821.

Ohrn, E. (2019). The effect of tax incentives on U.S. manufacturing: Evidence from state accelerated depreciation policies. Journal of Public Economics, 180, 104084. https://doi.org/10.1016/j.jpubeco.2019.104084

Opricovic, S. \& Tzeng, G.H. (2003). Defuzzification within a fuzzy multicriteria decision model, International Journal of Uncertainty, Fuzziness and Knowledge-based Systems. 11, 635-652

Park, J. (2016). The impact of depreciation savings on investment: Evidence from the corporate Alternative Minimum Tax. Journal of Public Economics, 135, 87-104. https://doi.org/10.1016/j.jpubeco.2016.02.001

Peles, Y. (1971). Rates of Amortization of Advertising Expenditures. Journal of Political Economy, 79-5.

Press, C., \& Davidson, S. (1964). The " Best " Tax Depreciation Method-1964. The Journal of Business, 37(3), 258-260.

Samaniego, R. M., \& Sun, J. Y. (2019). Uncertainty, depreciation and industry growth. European Economic Review, 120, 103314. https://doi.org/10.1016/j.euroecorev.2019.103314

Schinas, O., Grau, C., \& Johns, M. (2015). HSBA handbook on ship finance. In HSBA Handbook on Ship Finance. https://doi.org/10.1007/978-3-662-43410-9

Smith, V. L. (1963). Tax Depreciation Policy and Investment Theory. International Economic Review, 4(1), 8091.

Stickney, C. P. (1981). A Note on Optimal Tax Depreciation Research. The Accounting Review, 56(3).

Stopford, M., (2009). Maritime Economics. Routledge, New York.

Trading Economics. (2021). Baltic Exchange Dry Index. https://tradingeconomics.com/commodity/baltic Access: 02 May 2021.

Vogt, M., Pletsch, C. S., Morás, V. R., \& Klann, R. C. (2016). Determinants of goodwill impairment loss recognition. Revista Contabilidade e Financas, 27(72), 349-362. https://doi.org/10.1590/1808057x201602010

Wagenhofer, A. (2003). Accrual-based compensation, depreciation and investment decisions. European Accounting Review, 12(2), 287-309. https://doi.org/10.1080/0963818031000087835

Wakeman, L. M. D. (1980). Optimal tax depreciation. Journal of Accounting and Economics, 2(3), $213-237$. https://doi.org/10.1016/0165-4101(80)90003-8

Yussof, S. H., Isa, K., \& Mohdali, R. (2014). An Analysis of the Gap between Accounting Depreciation and Tax Capital Allowance in Malaysia. Procedia - Social and Behavioral Sciences, 164(August), 351-357. https://doi.org/10.1016/j.sbspro.2014.11.087 